\title{
Ultra-Low-Power Design and Hardware Security Using Emerging Technologies for Internet of Things
}

\author{
Jiann-Shiun Yuan*, Jie Lin, Qutaiba Alasad and Shayan Taheri \\ Department of Electrical and Computer Engineering, University of Central Florida, Orlando, FL 32816, USA; \\ ljie@knights.ucf.edu (J.L.); quitabaeng@knights.ucf.edu (Q.A.); shayan.taheri@knights.ucf.edu (S.T.) \\ * Correspondence: yuanj@mail.ucf.edu; Tel.: +2-1-407-823-5719
}

Received: 1 August 2017; Accepted: 5 September 2017; Published: 8 September 2017

\begin{abstract}
In this review article for Internet of Things (IoT) applications, important low-power design techniques for digital and mixed-signal analog-digital converter (ADC) circuits are presented. Emerging low voltage logic devices and non-volatile memories (NVMs) beyond CMOS are illustrated. In addition, energy-constrained hardware security issues are reviewed. Specifically, light-weight encryption-based correlational power analysis, successive approximation register (SAR) ADC security using tunnel field effect transistors (FETs), logic obfuscation using silicon nanowire FETs, and all-spin logic devices are highlighted. Furthermore, a novel ultra-low power design using bio-inspired neuromorphic computing and spiking neural network security are discussed.
\end{abstract}

Keywords: ADC; DPA; emerging technologies; hardware security; neuromorphic computing; side-channel attack; Trojans; tunnel FET; ultra-low power

\section{Introduction}

Advances in wired and wireless sensor networks have laid a solid foundation for the Internet of Things (IoT). It is estimated that around 30 billion IoT devices will be connected to the Internet by 2020 [1]. Examples of these devices include sensors, RFID tags, smart thermostats, and smart phones and gadgets. Those devices will be empowered to sense, process, and control the physical world events. Eventually, the IoT will lead us to the Internet of Everything (IoE), where the virtual world of information is integrated with the physical world of objects.

The Internet of Things incorporates devices from a very diverse background. These devices differ from each other in terms of their size, storage, energy consumption, computation, data rate, and other performance metrics. Seamless and interoperable communication among them is enabled via sensors and actuators embedded in them. These miniature sensors give a unique ID to each participating device in an IoT paradigm. Sensors broaden the scope and scalability of today's Internet by integrating them to the physical systems. However, it requires effort from the application developer's side because sensors are tiny, energy-starved, and constrained on computation and storage capacity. Designing secure solutions in the IoT system is difficult and complex due to the peculiar nature of the devices. Since sensors are computing-power-constrained and deployable from anywhere in the world, they are vulnerable to cyber attacks and have thus become the weakest link in the IoT system.

In this review paper, energy-constrained IoT devices for low-power design and security assurance are presented. Section 2 discusses key low-power design techniques for today's chip applications. Section 3 illustrates emerging technologies in logic and memory devices beyond CMOS (more than Moore). Steep sub-threshold slope transistors as well as resistive, phase change, and spin transfer torque (STT) memories are explained. Section 4 combines the near-threshold low-power technique using emerging tunnel FET (TFET) technology for logic gates and successive approximation register (SAR) analog-to-digital converter (ADC) designs. In addition, the noise shaping (NS) technique is 
adopted to increase the effective number of bits for the SAR ADC. Bio-inspired ultra-low-power neuromorphic computing for unsupervised learning and recognition is introduced in this section as well. Various hardware security issues are highlighted in Section 5. These include important encryption techniques, side channel attack/defense, logic locking/split manufacturing against reverse-engineering/counterfeiting, and camouflage layout. The uses of emerging technologies and lightweight encryption for correlation power analysis against side channel attack, silicon nanowire polymorphic gates, and all-spin logic devices for deception and logic locking, and a TFET secure SAR ADC design for Trojan countermeasures are shown in Section 6. Finally, a summary of this work is given in Section 7.

\section{Key Low Power Techniques in Digital, Analog, and Mixed-Signal Circuits}

\subsection{Digital Circuits}

Scaling of CMOS devices have continued for many decades to provide faster switching speed and lower power consumption. Numerous enabling approaches such as high- $\mathrm{k} /$ metal gate $[2,3]$ and FinFET $[4,5]$ have been used. Since the dynamic power dissipation of CMOS logic is proportional to the square of supply voltage $\mathrm{V}_{\mathrm{DD}}, \mathrm{V}_{\mathrm{DD}}$ scaling provides a way to constrain power dissipation of integrated circuits (ICs). However, when CMOS logic is operating at the sub-threshold voltage level, a significant increase in leakage power and circuit delay occurs [6]. Near threshold operation offers the optimization of power and performance tradeoff (see Figure 1). In addition, three-dimensional (3D) integration of IC using through silicon vias (TSVs) can enhance chip performance [7].

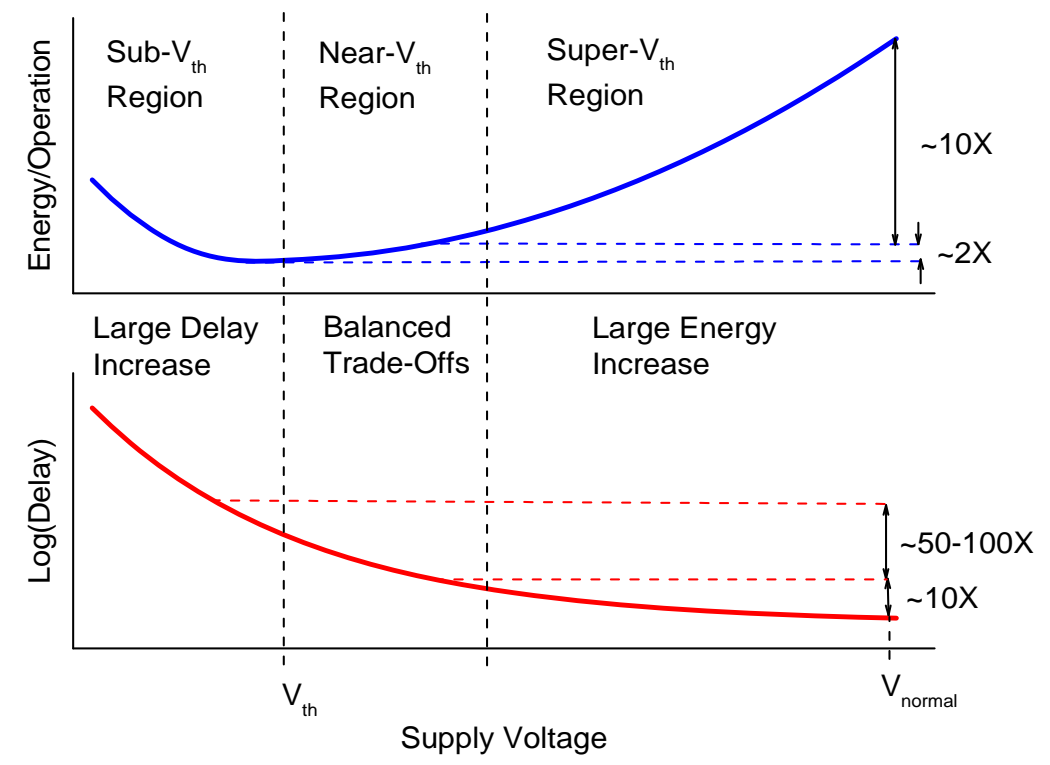

Figure 1. Energy and delay plots versus supply voltage scaling.

Energy efficiency is a major issue in modern digital systems. High computation demand has led academia and industry to provide architectural approaches for multicore and many-core systems that exploit system-wide power efficiency for a particular application domain. Power saving methods such as dynamic voltage and frequency scaling (DVFS) [8] is widely used in applications. DVFS scales the supply voltage and clock frequency based on the work load at run time. In DVFS, the power dissipation is controlled by adjusting the processor's voltage and frequency. Voltage and frequency scaling to offer power reduction has been implemented in commercial chips [9].

Multi-threshold (MT) CMOS technology provides a simple and effective power gating structure by utilizing high speed, low $V_{T}$ transistors for logic cells and low leakage, and high $V_{T}$ devices for sleep transistors [10]. Sleep transistors disconnect logic cells from the supply and/or ground to reduce 
the leakage in standby mode (see Figure 2). More precisely, multi-threshold CMOS uses low-leakage NMOS (PMOS) transistors as footer (header) switches to disconnect ground (power supply) from parts of a design in the circuit standby mode. There is a large amount of rush-through current from the power supply to ground when a multi-threshold CMOS circuit switches from sleep to active mode. On the other hand, when an MT CMOS circuit switches from sleep to active mode, it takes some time (wakeup latency) for the circuit to become functional and start working at its full performance level. Without some kind of always-on latches, the internal state of the MTCMOS circuit is lost when it is put into sleep mode. Because of the large amount of rush-through current and large wakeup latency for MTCMOS circuits, for short standby periods, it is better to put the circuit into an intermediate power-saving mode (called the drowsy mode). The reason is that the transition latency from the drowsy to active mode is much less than the wakeup time of the circuit when coming out of the sleep mode. Furthermore, if designed appropriately, drowsy circuits can retain a pre-standby internal state of the circuit. The downside of putting a circuit into drowsy mode is the higher amount of the leakage current compared to the case when the circuit is put into sleep mode.

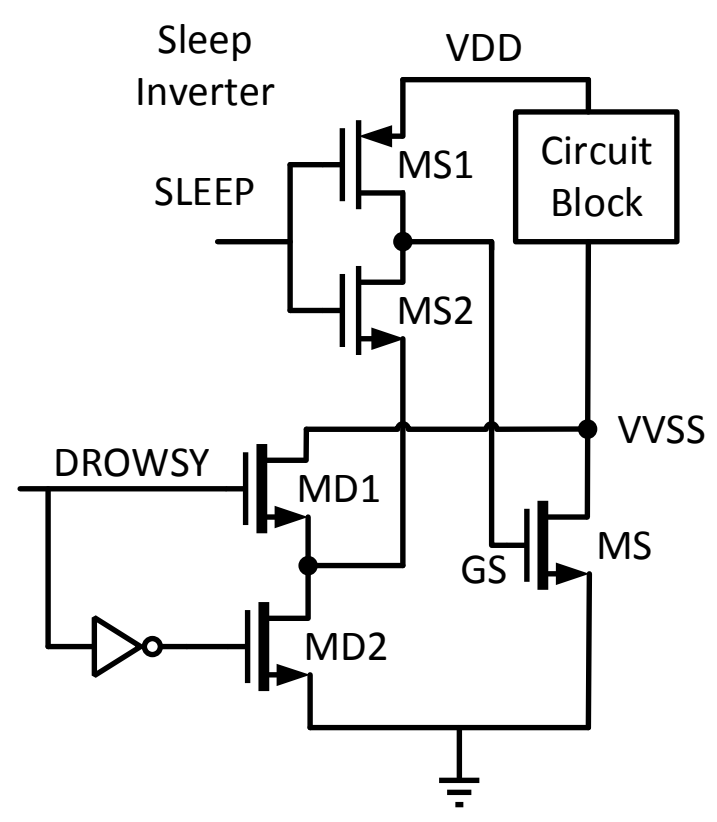

Figure 2. Implementation of sleep mode design.

In recent years, multi-core systems have become standard in the computer industry. The design of multi-cores takes advantage of thread-level parallelism in applications that are computationally intensive and highly parallel. Energy efficiency is one of the biggest challenges in the design of multi-core systems, and workload imbalance among parallel threads is one of the sources of energy inefficiency. DVFS thus can save energy consumption on multi-cores, but all of them assume that each core in a multi-core system contains only one hardware context and only one thread can execute on one core at a time. However, mainstream multi-core systems are moving to have simultaneous multi-threading (SMT) support in cores, and existing DVFS-based techniques are not effective to achieve maximum energy savings. A novel technique called thread shuffling, which combines thread migration and DVFS to achieve maximum energy savings and maintain performance on a multi-core system supporting SMT was proposed [11]. Thread shuffling is implemented and simulated in a cycle-accurate $\times 86$ multi-core system. The experiments show that it achieves up to $56 \%$ energy savings without performance penalty for selected Recognition, Mining, and Synthesis (RMS) applications from Intel Labs.

Other low-power design techniques include clocking gating [12], pipeline architecture [13], asynchronous signal transmission [14], and software and hardware co-design [15]. Asynchronous 
circuit design has long been a designer's interest. The advantages of asynchronous circuits include lower peak power dissipation, lower electromagnetic emission (EMI), free interchangeability of components between systems, and are more robust against temperature and process variations [16]. Asynchronous circuits, especially quasi-delay-insensitive asynchronous circuits, use local handshaking protocols in lieu of clocks to coordinate circuit behavior. The delay insensitivity and other unique features of quasi-delay-insensitive circuits allow for a more aggressive supply voltage scaling, implementing power gating without timing analysis or extra control overhead [17]. Asynchronous circuits connect multiple components effectively across a large die for energy efficiency.

Comparing various low-power design trade-offs or additional requirements, multi-threshold voltage technique requires the support of semiconductor process to make MOSFETs available with different threshold voltages. Asynchronous circuits may consume more chip area due to additional handshaking circuit components and dual rail encoding. Multi-core design requires parallel clock trees and needs additional interconnections on silicon among different cores. DVFS requires on-chip DC-DC converter for supply voltage scaling.

In addition to low-power mobile computing, energy saving in wireless communication is important for IoT applications. Clearly, energy efficient mobile computing requires an ultra-low-power system design [18]. Achieving a very low average power for a wireless system typically makes extensive use of duty cycling. The aim is to reduce the device "on" time to a short communication burst, and then between these active periods have the device enter a sleep mode to save power consumption.

\subsection{Analog Circuits}

Low-voltage operation in the analog circuit could be quite different from that of the digital circuit. For example, when the supply voltage is reduced to the near-threshold voltage of the MOSFET, the overdrive voltage (OV) or the voltage headroom is limited, which introduces a significant temperature shift of cutoff frequency of the MOS transistor and hence hinders the performance of the analog circuit. To address this temperature drift issue, Lin and Yuan [19] used an optimum overdrive voltage to reduce temperature sensitivity. With the mutual temperature compensation of carrier mobility and threshold voltage, the optimal bias point makes the cutoff frequency insensitive to temperature variation, as shown in Figure 3. A comparator using the optimum overdrive voltage technique is shown in Figure 4.

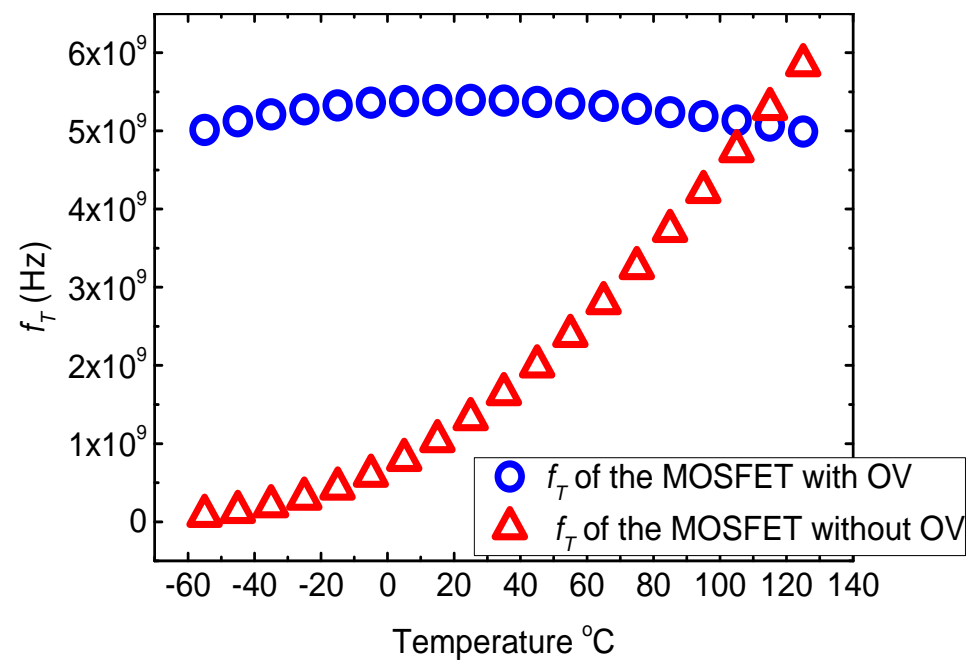

Figure 3. Cutoff frequency versus temperature. 


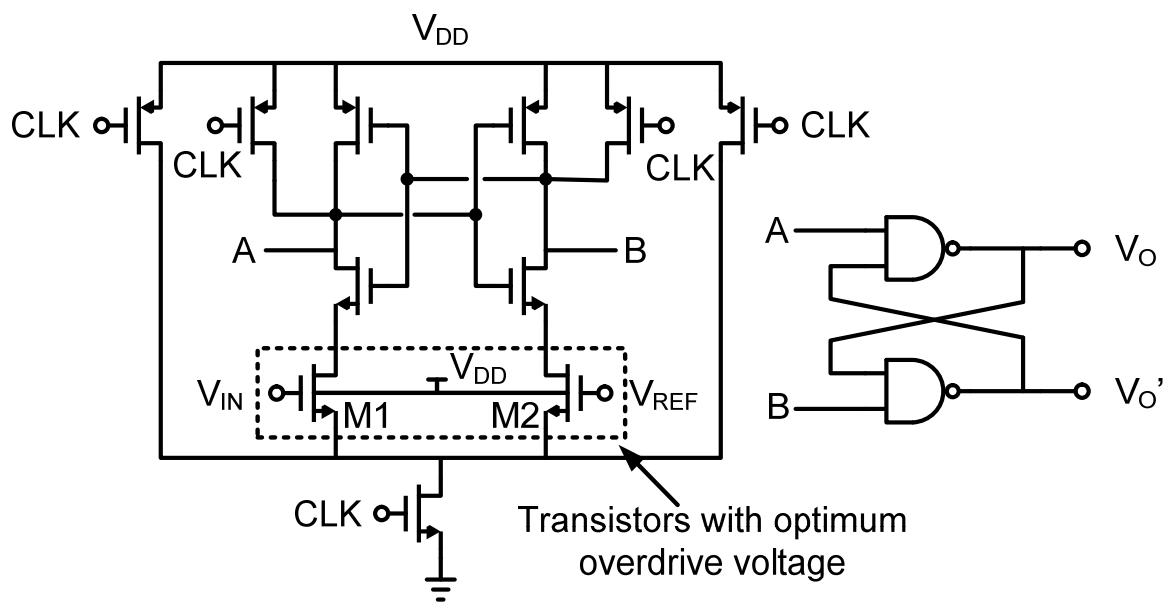

Figure 4. Schematic of the comparator using the optimum overdrive voltage technique.

\subsection{Mixed-Signal Circuits}

IoT devices that is deployed and accessed from any location and anytime require ultra-low energy for sensing, communication, and computing. An analog-to-digital converter is one of the essential building blocks for sensor interfaces that digitize the analog sensor output for subsequent digital signal processing. Most of the power supply of the sensor nodes-the harvesting devices such as solar cells—can only generate extremely low output voltage, usually less than $0.5 \mathrm{~V}$. Therefore, an ultra-low-voltage and low-power operation is critical for wireless IoT applications [20]. The output of the sensor usually needs to be processed by an ADC with moderate resolution and speed $(1-1000 \mathrm{kHz})$, while the signal level is also usually small [21]. In those low-power applications, ADCs are the most critical and power-hungry blocks. Furthermore, the use of TFETs can enhance the analog circuit performance [22].

A 6-bit SAR ADC topology for low supply voltage between 0.3 and $0.5 \mathrm{~V}$ (near threshold operation) was proposed in [23]. The single-ended structure has poor immunity to power supply noise and common-mode level drafting. Henceforth, a low-noise Low Drop Out (LDO) regulator and precise voltage reference are needed to guarantee the performance, which degrade the energy efficiency. In [23], a fully differential structure is introduced. The fully differential structure can not only provide twice the input and output swings of the ADC, which further improves the immunity against the supply noise by $6 \mathrm{~dB}$, but also cancel even-order distortion, which greatly improves the effective number of bits (ENOB) of the ADC. Figure 5 shows principal blocks of the 6-bits SAR ADC including the digital-to-analog conversion (DAC), comparator, and control logic. In Figure 5, $C_{i}=2 C_{i+1} ; C_{6}=C_{C}=5 \mathrm{fF}$, and the total capacitance used in the DAC is $640 \mathrm{fF}$. To make the maximum utilization of the supply voltage, the positive and negative voltage reference are VDD and GND, respectively, and $V_{C M}$ is VDD/2. Because of the fully differential operation, noise on the supply voltage can be cancelled out. Furthermore, a circuit that generate $V_{C M}$ can be coarse to reduce the area and power dissipation. The input signal is sampled through FET switches. In this design, feedback switches are also implemented using FET transistors to switch among VDD, GND, and $V_{C M}$. The comparator in Figure 6 is implemented based on a strong arm latch for low-power operation and generates the decision signal to control the SAR logic circuit. The SAR logic module comprises FET-based logic gates and generates the clock of all sampling switches and feedback switches.

The clock scheme for the SAR ADC is depicted in Figure 7a, where CLK is the external clock signal; CLK_COMP is the clock that triggers the comparator; $\mathrm{CLK}_{\mathrm{S}}$ is the sampling clock and $\mathrm{CLK}_{\mathrm{i}}$ is the clock that control the feedback switch of $C_{i}$, which is illustrated in detail in Figure $7 \mathrm{~b}$. The sampling period is 8 clock cycles, so there is enough time for the sampling circuit to settle. When the sampling clock is high, the comparator is disabled and the capacitor's bottom plate is connected to $V_{C M}$. When the 
sampling clock becomes low, the top plate of the capacitor array is isolated and the comparator begins to compare the voltage on them. $\mathrm{CLK}_{\mathrm{i}}$ will become high after the $i$ th decision is made and switches the bottom plate of $C_{i}$ to VDD or GND. In Figure $7 b, C L K_{i}$ is fed into a non-overlapping clock generation module to guarantee that the bottom plate of the capacitor $C_{i}$ will not be connected to both $V_{C M}$ and VDD (or GND) simultaneously. Signals $\mathrm{VS}_{\mathrm{VCMi}}, \mathrm{VS}_{\mathrm{VDD}}$, and $\mathrm{VS}_{\mathrm{GND}}$ are the control signals for the switches to connect the bottom plate of the capacitor $C_{i}$ to $V_{C M}, V D D$, and GND, respectively. $V_{C O M P}$ is the output voltage of the comparator and determines whether the bottom plate of $C_{i}$ is switched to VDD or GND.

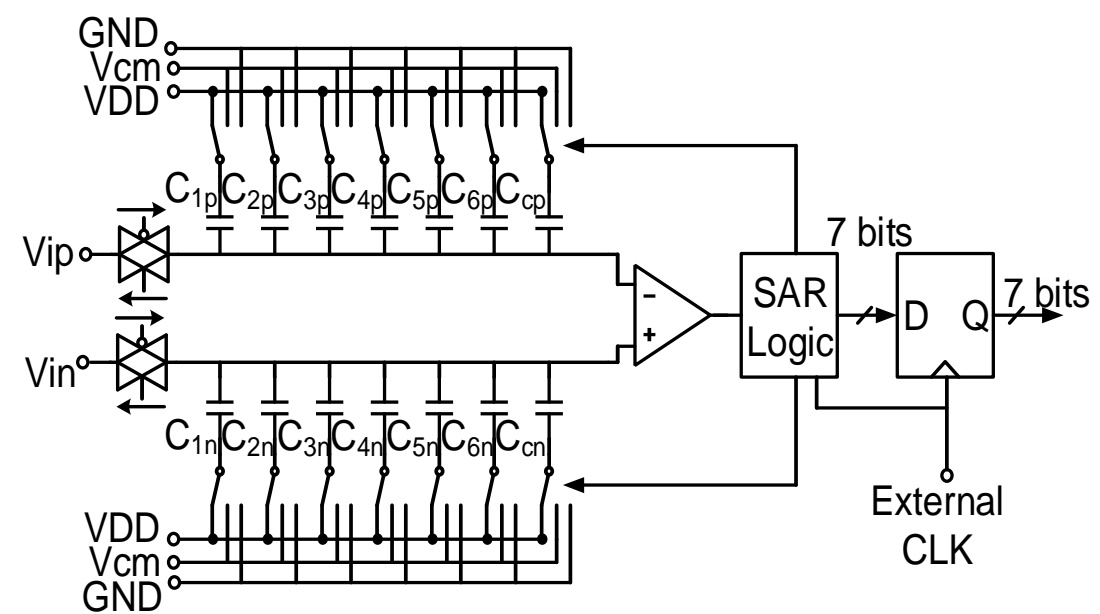

Figure 5. A successive approximation register (SAR) analog-digital converter (ADC) circuit schematic.

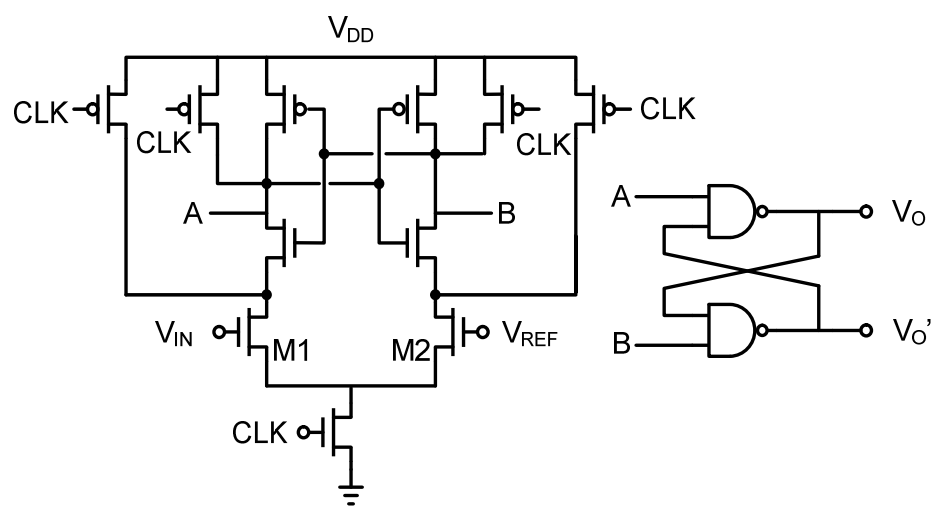

Figure 6. The comparator used in the SAR ADC.

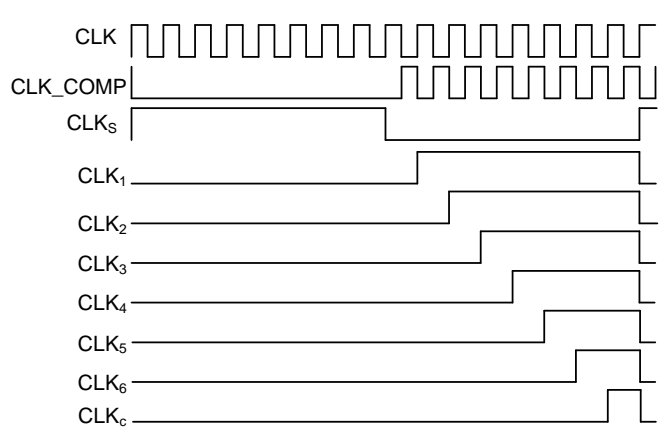

(a)

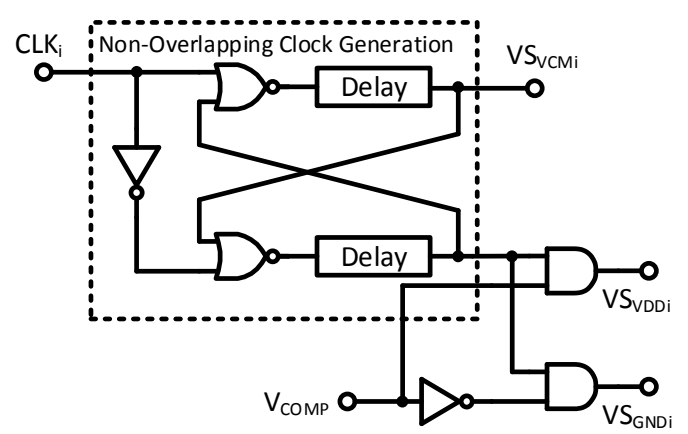

(b)

Figure 7. (a) Clock timing; (b) Clock generating logic. 
Because of the fundamental limitation and related secondary effects, the accuracy of SAR $\mathrm{ADC}$ is hard to achieve with a resolution over 10 [24]. The $k T / C$ noise is the main limitation of sampling accuracy. For moderate resolution ADCs, the minimum capacitance to achieve sufficient low sampling noise is usually larger than that required capacitance needed to yield adequate matching. Moreover, the number of unit capacitance evolves exponentially with the resolution of the ADC, leaving great difficulty for layout matching and parasitic reduction. To solve this problem, a common method is to use the oversampling technique to obtain a lower noise power spectral density in band. As an effective method to reduce quantization noise, noise shaping has been recently demonstrated in SAR ADCs $[25,26]$. However, in those works, the noise is only shaped to the first-order transfer function, leading to a setback of limited attenuation at low frequency and a smaller degree of freedom in parameter design. A 2nd-order noise shaping $\triangle \Sigma$ SAR ADC using TFETs can provide much less quantization noise than its first-order counterpart. By optimizing design parameters of the ADC, noise generated by the integrators is attenuated, leading to a decreased power consumption and silicon area.

The SAR ADC is a zero-order sigma-delta modulator without any form of noise shaping. Therefore, noise shaping can be realized by insert filters into the signal path [27]. The passive filters are a suitable choice for ultra-low-power, ultra-low-supply-voltage operation. Given the feedback path of the ADC is primarily defined by the SAR algorithm, feed-forward sigma-delta architectures are suitable for NS $\Delta-\Sigma$ SAR ADCs. Moreover, since the input signal to the loop filter is only the shaped quantization noise, the requirements on the linearity of the loop filter is greatly reduced. Henceforth, the influence of parasitic capacitances in the passive integrators is addressed by the feedforward architecture. The signal-flow graph of the second-order NS $\Delta-\Sigma$ SAR ADC [28] is shown in Figure 8.

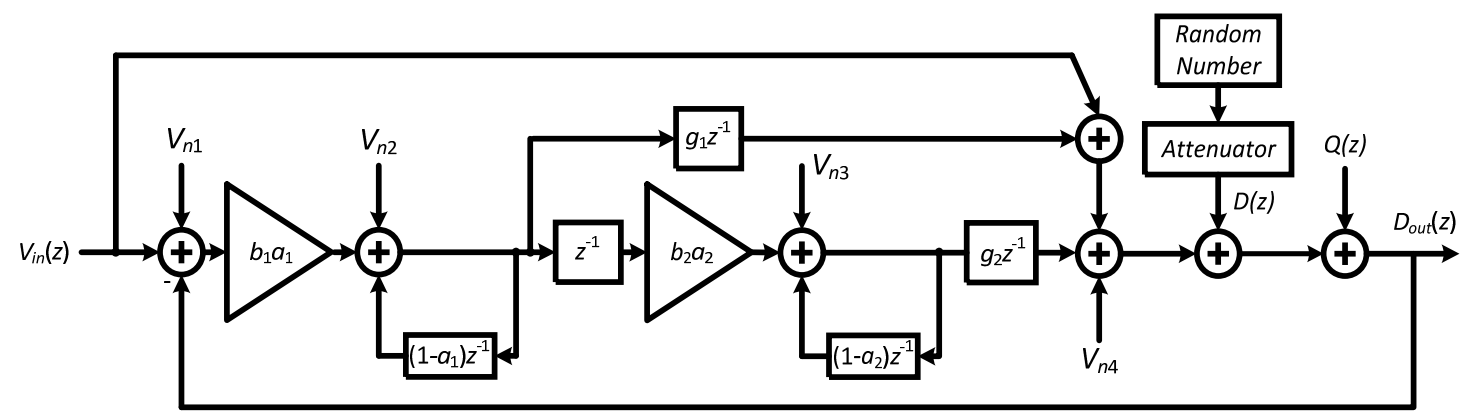

Figure 8. Signal flow diagram of the 2nd-order noise shaping (NS) $\Delta \Sigma$ SAR ADC.

The transfer function of the 2nd order NS $\Delta \Sigma$ ADC is

$$
D_{\text {out }}(z)=V_{\text {in }}(z)+\frac{\left[1-\left(1-a_{1}\right) z^{-1}\right]\left[1-\left(1-a_{2}\right) z^{-1}\right]}{1+A z^{-1}+B z^{-2}}[Q(z)+D(z)]
$$

where $Q(z)$ is the quantization noise, $D(z)$ is the dither signal, and $A$ and $B$ are given by

$$
\left\{\begin{array}{l}
A=-2+a_{1}+a_{2}+a_{1} b_{1} g_{1} \\
B=1-a_{1}-a_{2}+a_{1} a_{2}-\left(a_{1} b_{1}-a_{1} a_{2} b_{1}\right) g_{1}+a_{1} a_{2} b_{1} b_{2} g_{2}
\end{array}\right.
$$

The magnitude of noise transfer function (NTF) for the 2nd-order $\Delta \Sigma$ SAR ADC using $a_{1}=0.11$, $a_{2}=0.25$ is compared with previous published results in Figure 9. As seen in Figure 9, the 2nd-order noise shaping can offer an extra $19 \mathrm{~dB}$ attenuation at low frequency comparing to that of the first-order $\Delta \Sigma$ ADC result. 


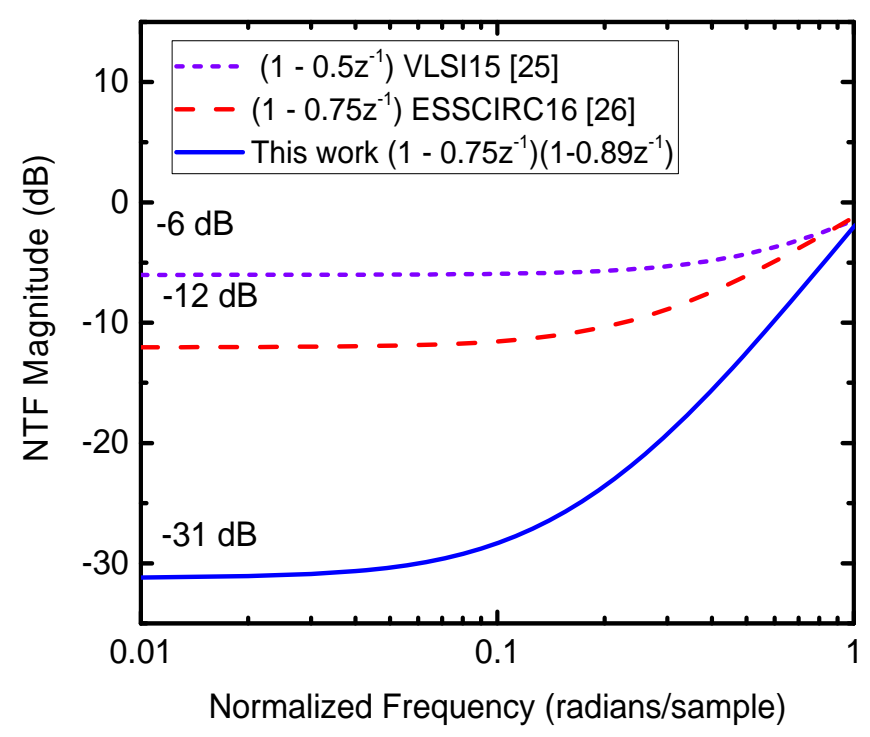

Figure 9. Different noise transfer function (NTF) performance versus normalized frequency.

Based on the principle of the proposed transfer function, a hybrid $\triangle \Sigma$ SAR ADC was implemented. The designed ADC comprises a 6-bit SAR ADC [23] and a second-order passive integrator. One extra switching of the DAC array Cc was added so that the residue is based on the full resolution of digital estimation. Moreover, the quantizer and the feedback DAC use the same capacitor array in the $\Delta \Sigma$ SAR ADC. Therefore, the DAC mismatch error transfer function (ETF) is always 1, and the mismatch error can be easily estimated and calibrated in the digital domain. The sampling frequency is $1.38 \mathrm{MHz}$ with the maximum input bandwidth of $43.1 \mathrm{kHz}$. The oversampling ratio (OSR) is 16 . The schematic of the ADCs is shown in Figure 10. In Figure 10, the clock generating circuit and SAR logic block is the main digital block of the circuit generating the control bits according to the output of the comparator.

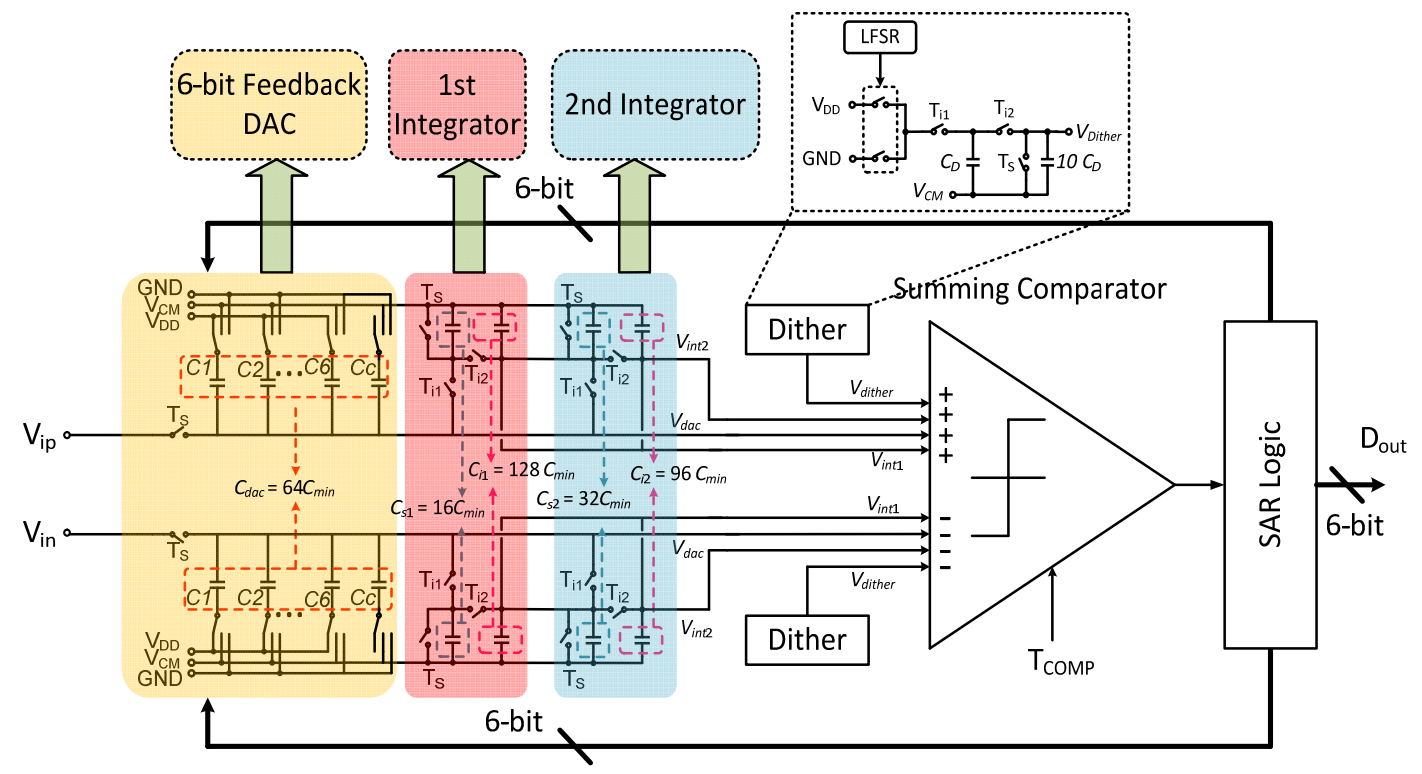

Figure 10. Second-order NS $\Delta-\Sigma$ SAR ADC with dither injection.

\section{Emerging Technologies}

Entering the smart society today, the amount of the information and data is growing explosively. Corresponding to the growth, demands for low-power, high-performance integrated circuits become 
even stronger. The slowdown of Moore's law intensifies the search of the next transistor and memory technologies beyond CMOS.

\subsection{Emerging Logic Devices}

\subsubsection{SiNW FET}

In several nanoscale FET devices, the superposition of n-type and p-type carriers is observable under normal bias conditions. The phenomenon, called ambipolarity, exists in silicon [29], carbon nanotubes (CNTs) [30], and graphene [31]. Through the control of this ambipolarity, we can adjust the device polarity. Transistors with a controllable polarity have already been experimentally demonstrated in carbon nanotube FETs [32], silicon nanowire (SiNW) FETs [33,34], and graphene FETs [35]. Given an additional gate, the operation of these FETs is enabled by the regulation of Schottky barriers at the source/drain junctions. The emerging device shown in Figure 11 is a stacked SiNW FET, featuring two gate-all-around (GAA) electrodes $[35,36]$. Stacked GAA silicon nanowires represent a natural evolution of FinFET structures and provides better electrostatic control over the channel and, consequently, superior scalability properties [36].

In the SiNW transistor, the Control Gate (CG) electrode acts conventionally by turning the device on and off, depending on the gate voltage. The second electrode, named the Polarity Gate (PG), is used to determine the transistor polarity dynamically between n-type and p-type. The input and output voltage levels are compatible, enabling directly cascadable logic gates [36,37]. Whereas many emerging devices demonstrate the polarity control property (SiNW FETs, graphene FETs, CNT FETs, etc.), SiNW FETs are process-compatible with the current silicon technology.

In Figure 11, when the input voltage of the PG is high, the SiNW transistor is an NMOS. When the voltage of the PG is low, it is a PMOS. Figure 12 displays its $I_{D}-V_{G}$ characteristics of the SiNW FET obtained from measurement. The nanowire stack has a $10 \mathrm{~nm}$ gate oxide, a $50 \mathrm{~nm}$ thick conformal polysilicon GAA structure, and an optimized distance $<20 \mathrm{~nm}$ for stacked nanowires. The advantages of using SiNW FETs for security implementation include their effectiveness in camouflage layouts against reverse-engineering and polymorphic gates for logic obfuscation (see Sections 6.2 and 6.3 for details).
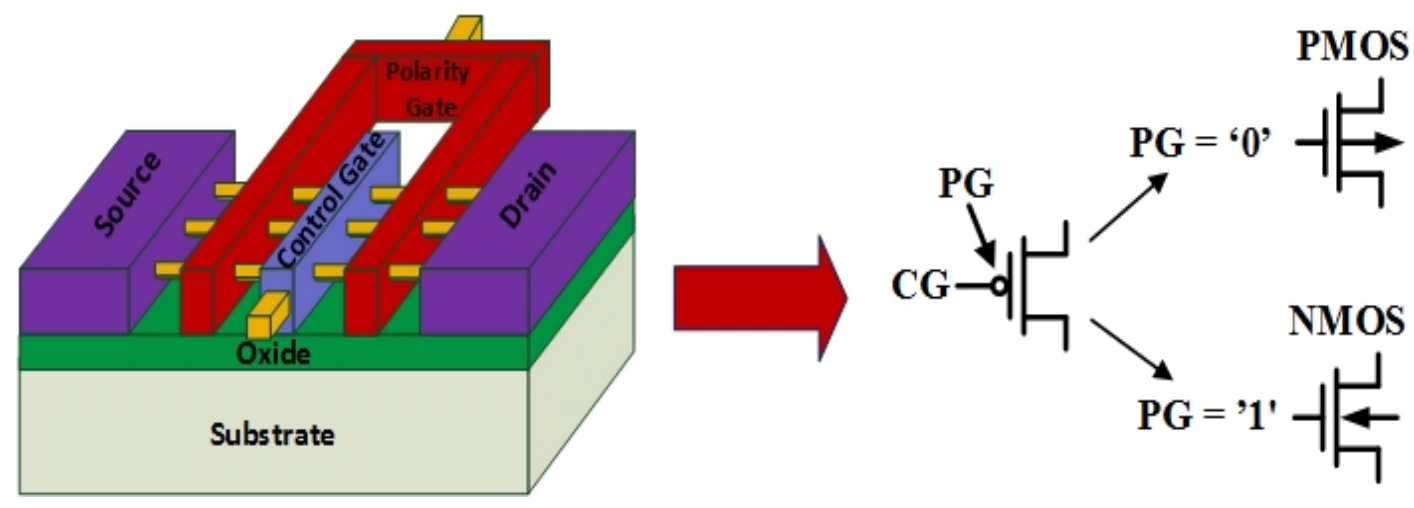

Figure 11. Schematic illustration of silicon a silicon nanowire transistor. 


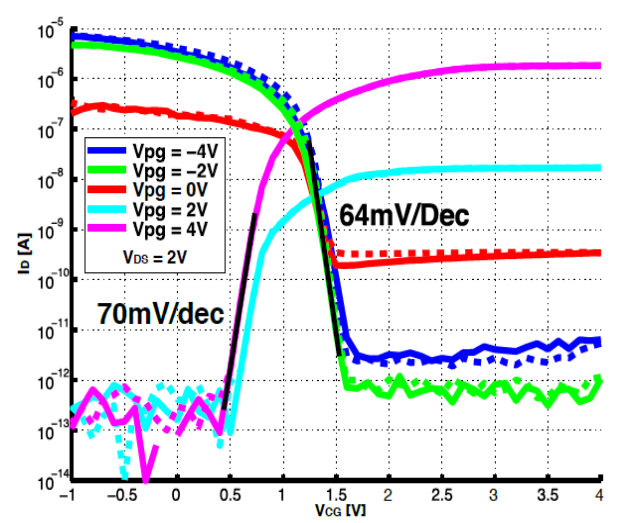

Figure 12. The drain current versus gate-source voltage. Reproduced with permission from [36], Copyright IEEE, 2012.

\subsubsection{Graphene SymFETs}

As MOSFET alternatives, tunneling-based transistor technologies [38,39] have been actively pursued. Among these devices is a double-layer graphene transistor-often referred to as a SymFET [40]. In the SymFET device, tunneling occurs between the two graphene sheets that are separated by insulating and oxide layers. Possible $\mathrm{I}_{\mathrm{DS}}-\mathrm{V}_{\mathrm{DS}}$ characteristics of a SymFET, which are a function of a top-gate voltage $\left(\mathrm{V}_{\mathrm{TG}}\right)$ and back-gate voltage $\left(\mathrm{V}_{\mathrm{BG}}\right)$, are illustrated in Figure 13 (see the device symbol in the inset). Similar characteristics have also been observed experimentally [41]. More specifically, $\mathrm{V}_{\mathrm{TG}}$ and $\mathrm{V}_{\mathrm{BG}}$ change the carrier type and density of the drain and source graphene layers by an electrostatic field to modulate $\mathrm{I}_{\mathrm{DS}}$. As seen in Figure 13, the value and position of the peak current depends on the $\mathrm{V}_{\mathrm{TG}}$ and $\mathrm{V}_{\mathrm{BG}}$. Note that the $I-V$ characteristics shown in Figure 13 assume a SymFET device with a $100 \times 100 \mathrm{~nm}$ footprint and an insulating layer of boron nitride that is 1.34-nm-thick. Tuning the insulator thickness could represent another design capability. For example, theoretically, by reducing barrier thickness to two layers of boron nitride, the tunneling current is increased substantially at the expense of leakage current [42]. The unique $I-V$ characteristics of SymFET offer some interesting circuit-level alternatives for realizing both analog and digital circuits [42,43]. For example, cascading SymFET devices leads to an extremely small majority gate design. Furthermore, different combinations of $\mathrm{V}_{\mathrm{TG}}$ and $\mathrm{V}_{\mathrm{BG}}$ can change the shape of the $I-V$ curves significantly. The unique property of SymFETs may be used for hardware security such as the prevention of supply voltage-based fault injection.

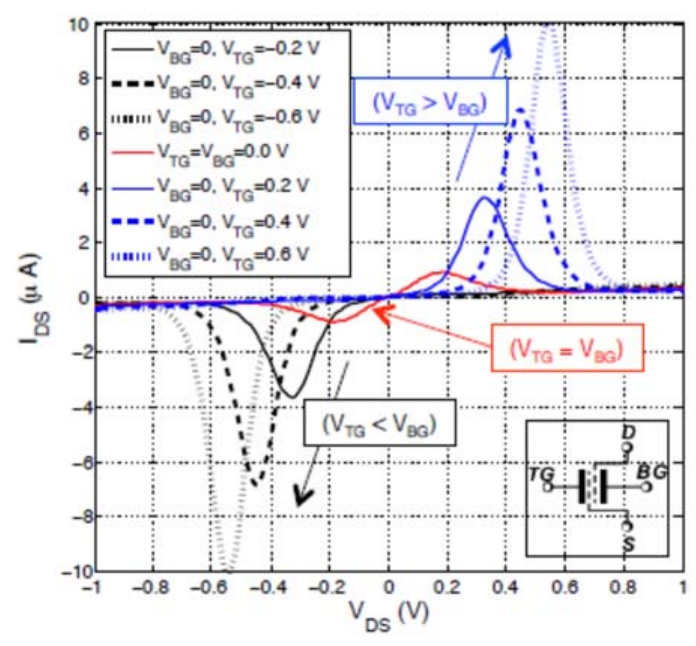

Figure 13. $I-V$ characteristic of a SymFET. 


\subsubsection{Tunnel FET}

For a FET operating, a potential barrier that separates the source from the drain is modulated by the gate voltage. Carriers from the source are injected into the channel that have an energy higher than the potential barrier. Since a change of potential barrier will sample the Boltzmann tail of the Fermi distribution of the carriers in the source, the sub-threshold slope is limited to $60 \mathrm{mV} / \mathrm{dec}$ at room temperature. To overcome this restriction, band-to-band tunneling [44] offers such a solution. The probability of carriers tunneling from the valence band to the conduction band of a semiconductor depends on the alignment of the band edges. In contrast to the conventional FET, the tunnel FET will not sample the Boltzmann tail of the distribution function but rather sharply turn on when the band edges are aligned properly for the tunnel process to kick in. Thus, the tunnel FET can turn on the device at a rate smaller than $60 \mathrm{mV} / \mathrm{dec}$.

Tunnel FETs utilize a gate voltage to control the band-to-band tunneling across a P-N junction. The cross-section and energy band diagrams of n-channel TFET in the OFF and ON states are shown in Figure 14a,b. As seen in Figure 6, when a zero bias voltage is applied to the gate of the TFET, the conduct band minimum of the channel $\mathrm{E}_{\mathrm{C}}$ is above the valence band maximum of the source $\mathrm{E}_{\mathrm{V}}$. Thus, the band-to-band tunneling is not possible and the device is cut off. When a positive bias voltage is applied to the gate of the $\mathrm{n}$-channel transistor, the conduction band of the channel is shifted down. A tunneling window, $V_{T W}$, will be created if $E_{C}$ is below $E_{V}$. As a result, electrons in the source will tunnel into the channel and the device is on.

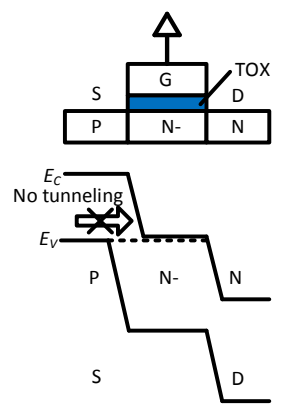

(a)

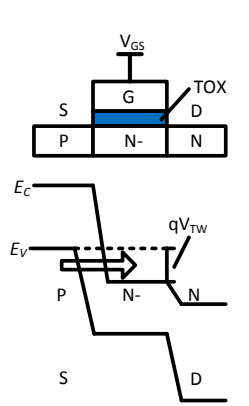

(b)

Figure 14. (a) Tunnel field effect transistor (TFET) in the cutoff mode; (b) TFET is turning on.

Figure 15 shows the drain current versus gate-source voltage for silicon FinFET and III-V heterojunction TFET. The TFET exhibits a steeper sub-threshold slope than that of the FinFET. Steep sub-threshold slope transistors are more favorable for low-voltage and low-power electronics. The advantages of TFETs include low-voltage and low-power operation (see Section 4 for detail) and lightweight encryption (see Section 6).

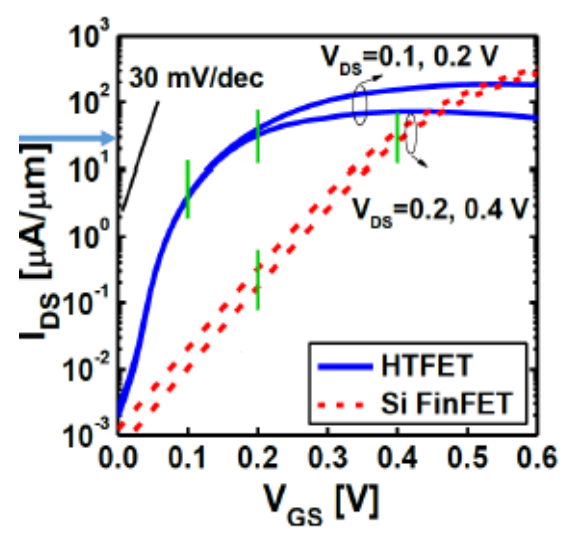

Figure 15. Drain-source current versus gate-source voltage. 


\subsubsection{Ferroelectric FET}

The conventional gate dielectric can be replaced by an insulator that provides an effective negative capacitance (NC). NC causes the differential potential drop in the semiconductor and the insulator to have opposite polarity, enabling MOS current to increase at a rate much better than $60 \mathrm{mV} /$ dec. Ferroelectric (FE) insulators had been predicted to have NC in accordance with the Landau mean-field-based theory [45].

Negative capacitance due to the addition of an FE material to the insulator stack has been demonstrated via experiment [46]. Hysteretic switching with steep slope FE FETs with $\mathrm{PbZr}_{0.52} \mathrm{Ti}_{0.48} \mathrm{O}_{3}$ (PZT) and hafnium dioxide $\left(\mathrm{HfO}_{2}\right)$ as the composite gate insulator has been reported [47]. FE FETs were fabricated on p-type silicon substrate with a doping concentration of $5 \times 10^{16} \mathrm{~cm}^{-3}$. A 10-nm-thick $\mathrm{HfO}_{2}$ was deposited underneath the PZT film via atomic layer deposition (ALD) to prevent reaction between the PZT and the silicon channel directly (see Figure 16). Note that ferroelectric FET here is built on top of the conventional CMOS process. Measured $\mathrm{I}_{\mathrm{DS}}-\mathrm{V}_{\mathrm{GS}}$ characteristics of the FE FET shows a steep sub-threshold turn-on, with a slope of about $13 \mathrm{mV} / \mathrm{dec}$.

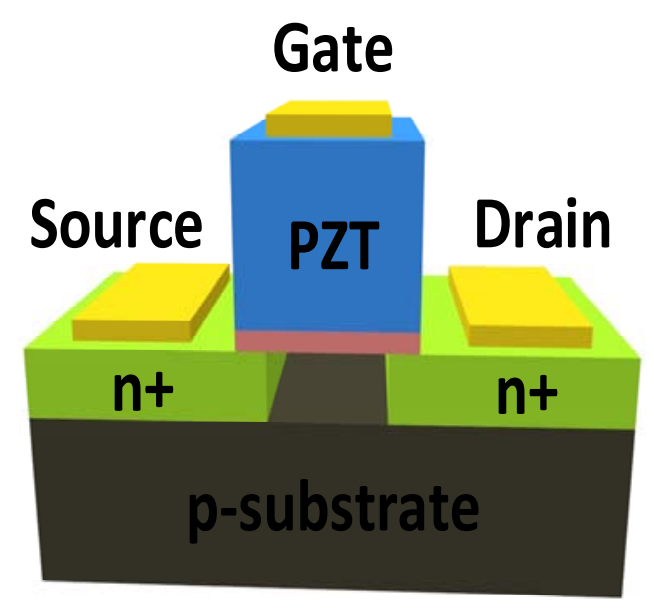

Figure 16. Schematic of a ferroelectric (FE) field effect transistor (FET).

In addition, vanadium dioxide $\left(\mathrm{VO}_{2}\right)$ exhibits an electrically induced abrupt insulator to metal transition. Phase-transition FET on silicon substrate, based on recent experimental data, can produce a deep sub-threshold slope of $8 \mathrm{mV} / \mathrm{dec}$ [48].

\subsection{Emerging Memories}

Static RAMs (SRAMs) and dynamic RAMs (DRAMs) are dominant memory technologies today due to their high speed, manufacturability, and scalability. Six-transistor SRAMs are widely used in high performance L1 and L2 cache arrays, while DRAMs are used as off-chip memory arrays or as embedded DRAMs (eDRAMs) as high density caches. In SRAMs and DRAMs, data are stored as charges in bit-cells. More energy is required to maintain data in SRAMs and DRAMs cells due to increasing leakage in scaled transistor dimensions.

Emerging non-volatile memories (NVMs) such as magnetic tunnel junction (MTJ), spin-transfer torque RAMs (STT-RAMs), resistive RAMs (RRAMs), and phase change memories (PCMs) were developed to replace or complement SRAMs and DRAMs to increase memory bandwidth and reduce leakage power density. Magnetic materials store information in terms of up and down spins. Using the energy barrier, magnets can retain spin information in a non-volatile fashion. The non-volatile nature suggests that memories using magnets do not need to be constantly powered. Ideally, NVMs have no standby power consumption. 


\subsubsection{Resistive Memory}

A RRAM cell typically consists of an insulator between top and bottom electrodes. When a set (positive) voltage is applied, a conductive filament (CF) in the insulator is formed due to the redistribution of oxygen vacancies. The RRAM resistance thus decreases to a Low Resistance State (LRS). When a reset voltage (opposite polarity) is applied, the CF ruptures, the RRAM resistance enters a High Resistance State (HRS). Figure 17a shows the schematic of a TiN/HfOx/Si-based RRAM cell [49]. The Al/Ti/TiN serves as the top electrode and $\mathrm{n}^{+} \mathrm{Si}$ serves as a bottom electrode. The HfOx is the insulator filament with a very thin $\mathrm{SiO}_{2}$ interfacial layer. When a positive SET voltage is applied, a CF forms in the HfOx layer connecting $\mathrm{TiN}$ and $\mathrm{SiO}_{2}$ due to the generation of oxygen vacancies $\mathrm{V}_{\mathrm{O}}$ [35]. Therefore, the device switches from HRS to LRS. During the RESET process (where a negative supply voltage is used), the recombination of oxygen vacancies and oxygen ions leads to the rupture of CF. Hence, the device switches from LRS to HRS. Figure 17b shows the resistance distribution of LHS and HRS over 100 continuous DC sweep cycles. It is worth pointing out that in 2015 SanDisk signed a long-term partnership with Hewlett Packard to co-develop RRAM technologies and expects products to enter the enterprise storage market by 2018 [50].

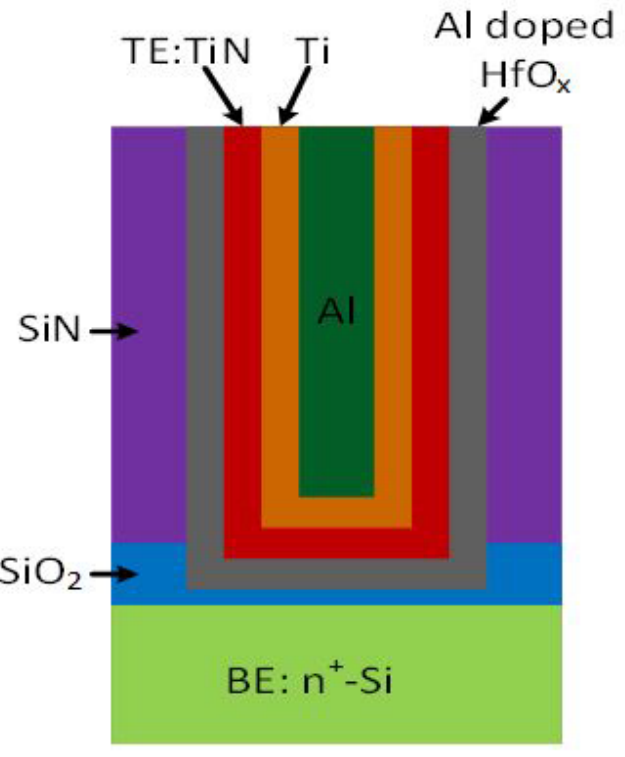

(a)

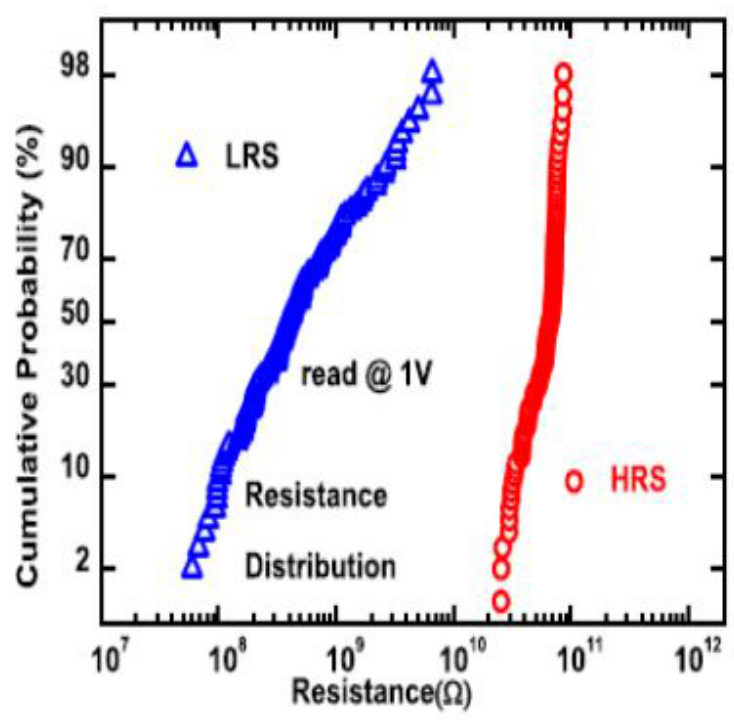

(b)

Figure 17. (a) A resistive RAM (RRAM) cell cross-section; (b) measured resistance distribution of $\mathrm{R}_{\mathrm{HRS}}$

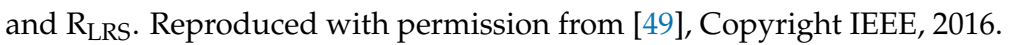

\subsubsection{Phase Change Memory}

Phase change memory [51] employs a reversible change of electrical resistivity in different phases to store data. A PCM storage cell is comprised of a layer of chalcogenide (an alloy of germanium, antimony, and tellurium) sandwiched between two electrodes and a heating resistor extended from one of the electrodes to contact the chalcogenide layer, as shown in Figure 18 [52]. The phase change of chalcogenide is induced by intense localized Joule heating. In the melted amorphous phase, the material exhibits high resistivity because of the disordered crystalline lattice, which can represent a binary " 0 ". In the frozen polycrystalline phase, the chalcogenide exists in a regular crystalline structure and exhibits low resistivity, which can represent a binary " 1 ". PCM offers many advantages such as scalability and low standby power dissipation [53]. 


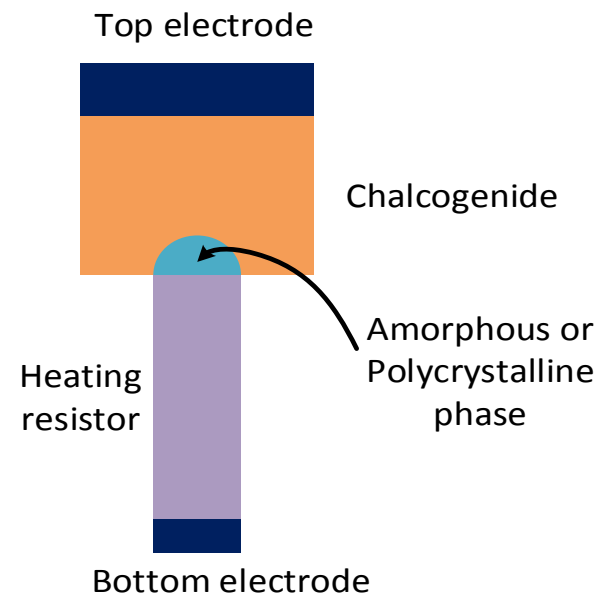

Figure 18. A basic phase change memory cell structure.

It is known that DRAM has been the building block for computer systems during the past 40 years. As DRAM faces increasingly severe scalability and power consumption issues, PCM is a promising alternative to DRAM. In 2016, IBM Research reliably demonstrated the storage of 3 bits of data per cell using a phase-change memory technology that could help transition electronic devices from standard RAM and flash to a much faster and more reliable type of storage [54]. In addition to its non-volatility and energy saving, PCM has a high-density property and sustainable scalability. However, PCM's storage cell can only endure a limited number of writes. The wear-leveling mechanism must be applied to prevent cells from being worn out sooner than others. Traditionally, an address mapping table like that used in flash memory can be employed for wear-leveling [55]. The table-based wear-leveling techniques, however, are not suitable for PCM because of the intrinsic differences between PCM and flash memory. Algebraic-mapping-based wear leveling [56] was proposed to leverage an algebraic algorithm to calculate the mapping between the logical address and the physical address, instead of looking for the mappings in a table. The detail of PCM security is discussed in Section 6.4.

\subsubsection{Spin Transfer Torque Memory}

The spin-based memories that are considered as the next generation of memory technologies are built upon the principles of spintronics. The uniqueness of these memories is in the use of the degree of freedom of the electron spin for computation, and their advantages over the traditional memories (such as CMOS-based DRAMs) are mainly energy efficiency, scalability, density, and speed. The spin-based devices can hold information even when they are off since the magnetic material inside them is able to hold the information with no connection of supply voltage. With this feature, these devices' leak much less current and make it possible to integrate a greater number of them on the on-chip last level cache. Additionally, the compatibility of the spin-based logic devices with the transistor-based devices provides an opportunity to construct a hybrid computing system. The most prominent spin-based devices are spin-transfer torque random access memory and domain wall memory (DWM). An STT-RAM based cache provides an inherent trade-off between write latency and read latency. A typical transistor and magnetic tunnel junction (MTJ) cell is shown in Figure 19a [57]. The magnetic tunnel junction is the basic storage device in the spintronic field that provides data non-volatility, fast data access, and low-voltage operation. Each MTJ consists of two ferromagnetic layers separated by a very thin tunneling oxide. Magnetization in one of the layers (referred to as the pinned layer) is fixed in one direction. The other ferromagnetic layer (referred to as the free layer) is used for information storage [58] (see Figure 19b). Data writing is performed by using the spin-polarized current to change the magnetic orientation of the free layer with respect to the fixed layer in the MTJ device. The junction resistance is low (" 0 " state) when the two layers are spin-aligned (parallel state) and is high ("1" state) when the two layers are in opposite directions (anti-parallel state). 
The cell can be read by applying a small bias voltage and sensing the current. The characteristic of the MTJ magnet can be captured using the Tunneling Magneto Resistance (TMR) defined by

$$
T M R=\left(\frac{R_{A P}-R_{P}}{R_{P}}\right) \times 100 \%
$$

where $R_{A P}$ is magneto resistance for the anti-parallel state and $R_{P}$ is the magneto resistance for the parallel state. The MTJ can be integrated with CMOS using 3-D technology. IBM demonstrated a $128 \mathrm{~kb}$ MTJ-based MRAM in 2003, showing that MRAM performance can be better than that of DRAMs [59]. In this work, the MTJ security is discussed in Section 6.4. Furthermore, it is worth pointing out that Everspin Technologies has developed the DDR memory products using the spin transfer torque technology in the market place [60].

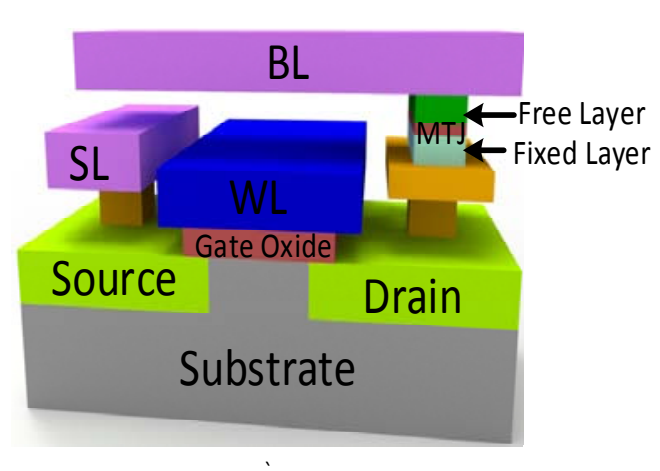

(a)

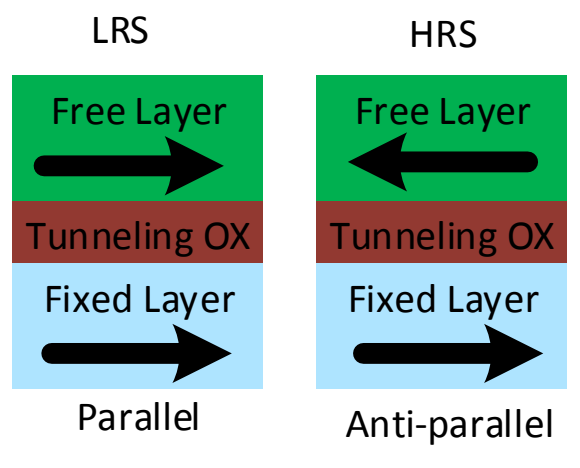

(b)

Figure 19. (a) A 3D plot of a magnetic tunnel junction (MTJ) with a pass gate transistor; (b) free layer to fixed layer orientation of a magnetic tunnel junction.

\subsubsection{Domain Wall Memory}

The spin-transfer torque random access memory and domain wall memory (DWM) are the key representations in spintronics, especially due to their multi-level cell (MLC) capability in breaking the memory density barrier. The racetrack memory (RM) was proposed first by Parkin et al. in 2008 [61]. The first demonstration of the RM wafer with its fabrication in IBM $90 \mathrm{~nm}$ CMOS technology was performed by Annunziata et al. in 2011 [62]. The application of this wafer for the regular on-chip caches [63] and the on-chip general purpose graphic process unit (GPGPU) caches [64] were also explored. DWM generally includes three parts: write head, read head, and magnetic nanowire (NW). Similar to the terminals of magnetic layers in the conventional magnetic tunnel junction, the read and write heads of DWM hold the bits in the form of magnetic polarity. According to this memory structure, a domain wall is created between the domains of opposite polarities in the nanowire. In order to shift the domain walls (or the corresponding bits) forward and backward, a charge current is injected from the contacts at either the left or the right side of the nanowire. This behavior is similar to that seen from a shift register. Therefore, for reading (or writing) a certain bit in the nanowire, its position is brought under the read (or the write) head through a current injection and then changing (or sensing) the MTJ resistance. A racetrack domain wall memory structure can be seen in Figure 20 [65]. 


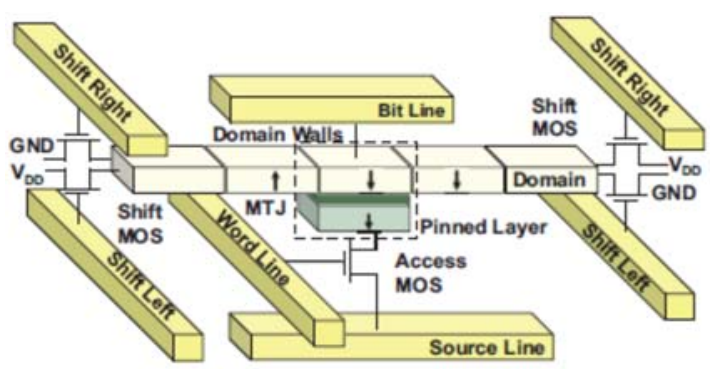

Figure 20. A racetrack domain wall structure.

\subsubsection{All-Spin Logic}

All-spin logic (ASL) device includes the nanomagnetic unit, which is used to store the binary data, an isolation layer between the input (with low spin polarization factor) and output (high spin polarization factor) ports, and one non-magnetic channel. Figure 21 shows a simple ASL with two magnets [66]. These two magnets are polarized in the same direction and connected with each other through a non-magnet channel. The channel is made from nickel or copper due to the high spin-flip length. The maximum length of the channel is reliant on the spin-flip length, which is used to identify the maximum distance that the spin current can travel. On applying negative VDD, the spin current will flow from M1 (where M is magnet) through the channel. The charge current will flow from GND to VDD, and the electrons will flow from VDD to GND. Spins in the same direction of M1 will pass, while spins in the opposite direction will not pass M1 (electrons are filtered). Since the output of M1 has high spin polarization and the input of M2 has low spin polarization, M1 will dominate the spin current, and the passed spins will accumulate in the channel. Meanwhile, M2 will receive a large spin current from M1. The direction of M2 will not change because both M1 and M2 have the same magnetization direction. Therefore, the whole design will work as a buffer. In contrast, on applying positive VDD, the electrons will flow from the ground to the M1. As a consequence, spins in the opposite direction of the magnet will accumulate in the channel. Meanwhile, only the spins in the same direction as M1 will pass out of M1, while the spins in the opposite direction will move through the channel to switch the direction of M2, so the device will work as an inverter [67]. Based on this phenomena, one leverages the Current-In-Plane non-local spin valve modular model in [68] to simulate the all-spin logic. One can design a simple ASL with two magnets to obtain a simple polymorphic gate (inverter/buffer). We can easily switch the functionality from buffer to inverter (by supplying positive VDD) or from inverter to buffer (by supplying negative VDD). An input voltage of $50 \mathrm{mV}$ (positive VDD) is applied to invert the direction of M2. It is worth noting that the designer can easily improve the switching speed by increasing the input voltage at the expense of increased power dissipation. Therefore, it is a trade-off between the delay and energy consumption [69]. The feature of the ASL device might provide robust IP protection against several attacks with less performance overhead. The detail of ASL security implementation for logic locking is presented in Section 5.5.

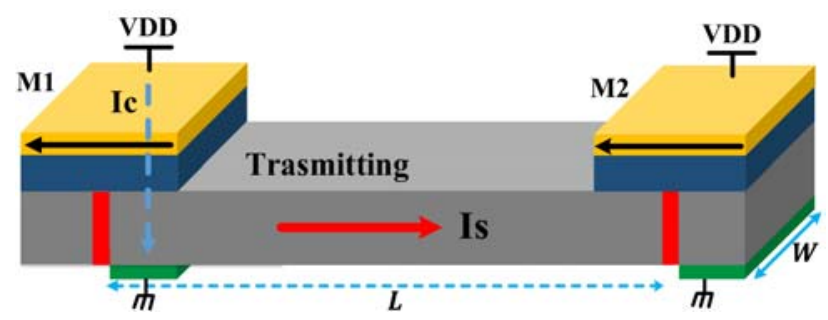

Figure 21. A simple all-spin logic (ASL) with two magnets. 


\section{Ultra-Low-Power Design Using Emerging TFET Technologies}

For the emerging transistor technologies discussed in Section 3.1, TFET technology may be more promising than its NC FETs, SymFETs, and ferroelectric FETs counterparts for low-voltage, low-power electronics applications.

\subsection{Digital Logic and Circuits Using TFETs}

Today, we are entering a "more than Moore" world, where computing is used for a multitude of applications including high-end servers, mobile computing devices, and pervasive sensor motes. Those make energy efficiency critical. As discussed in Section 2, supply voltage scaling in the near-threshold region provides optimal energy efficiency. Figure 22 shows the energy versus delay plot for CMOS and TFET AND gates subjected to different supply voltage levels. For the supply voltage ranging from 0.2 to $0.5 \mathrm{~V}$, the TFET logic gate - AND operation exhibits a much better energy and delay performance than its CMOS counterpart. Similar energy-delay characteristics can be observed between TFET and CMOS adders and L1 cache [70].

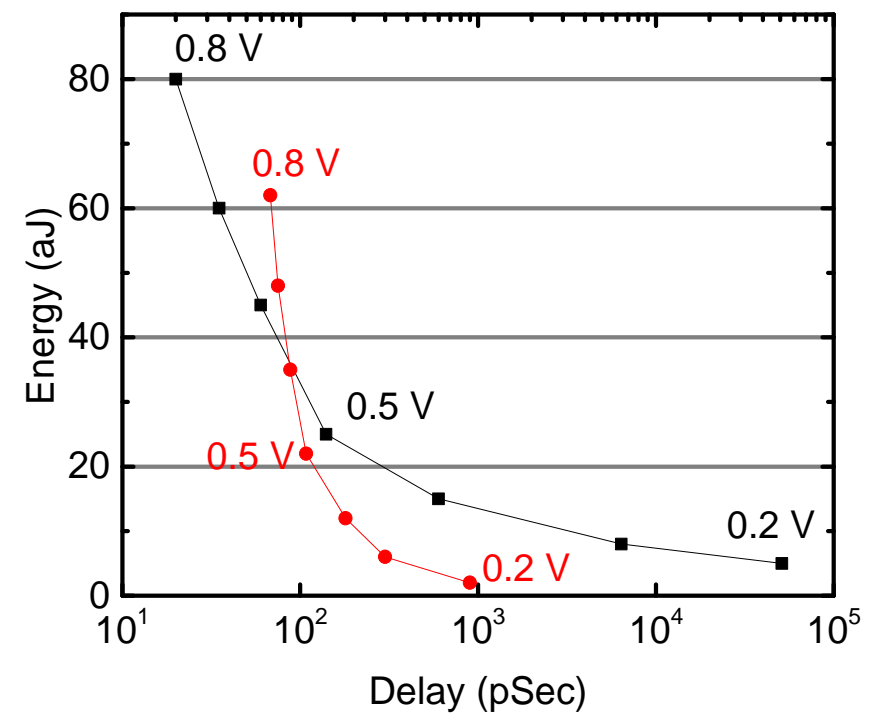

Figure 22. Energy versus delay for CMOS (squares) and TFET (circles) logic gates-AND operation.

\subsection{Low-Power, Low Voltage SAR ADC Using Emerging TFET Technology}

Transistor-level simulation of the TEFT based ADC is performed using Cadence ${ }^{\circledR}$ Spectre ${ }^{\circledR}$ (San Jose, CA, USA) with a modified Verilog-A transistor model for TFET transistor. The Verilog-A model use the Kane-Sze formulas [71] that capture the essential features of the tunneling current including bias-dependent subthreshold swing, super-linear drain current onset, and ambipolar conduction. A $20 \mathrm{~nm}$ CMOS-based ADC is also designed by replacing all TFET transistors with $20 \mathrm{~nm}$ CMOS transistors with $20 \mathrm{~nm}$ PTM-MG SPICE model [72]. This CMOS-based ADC is also simulated using Cadence ${ }^{\circledR}$ Spectre ${ }^{\circledR}$ [73] to compare the performance of TFET and CMOS technology. The full range inputs to the ADC are two sinusoid waves of peak-to-peak value of VDD, and the phase difference is $180^{\circ}$, making the differential-mode peak-to-peak value of full range input signal 2 VDD. The minimum TFET transistor length is $20 \mathrm{~nm}$. Both the Verilog-A model for TFET and PTM-MG model for CMOS include parasitic gate-source and gate-drain capacitances [74]. The oxide thickness for the TFET is $2 \mathrm{~nm}$.

To compare the performance of TFET and $20 \mathrm{~nm}$ CMOS technology, both TFET-based ADC and CMOS-based ADC are evaluated for the Effective Number of Bits (ENOB) and energy. Figure 23 depicts the ENOB of both TFET-based and CMOS-based ADCs. As shown in Figure 23 that, when power supply increases, the ENOB of the TFET-based ADC increases rapidly and saturates at 5.8 bits 
when VDD is above $0.5 \mathrm{~V}$. At a same supply voltage, the TFET-based ADC shows better ENOB than that of CMOS-based ADC. CMOS-based ADC also stops to work when VDD $\leq 0.3 \mathrm{~V}$ due to large on-resistance for CMOS transistor. A thorough comparison between TFET ADC and reported CMOS ADCs in the literature $[23,24]$ is made, and the results are displayed in Figure 24. To explore the TFET benefits in the sub-threshold region, we set the VDD at $0.3 \mathrm{~V}$ and the simulation temperature at $25{ }^{\circ} \mathrm{C}$. The power dissipation of the ADC is measured in terms of energy, which is defined as Energy $=$ Power/Sampling Frequency. Based on Figure 24, the simulated TFET-based SAR ADC is one to three orders of magnitude more energy-efficient than that of most fabricated CMOS ADCs and three times better than state-of-the-art CMOS ADC.

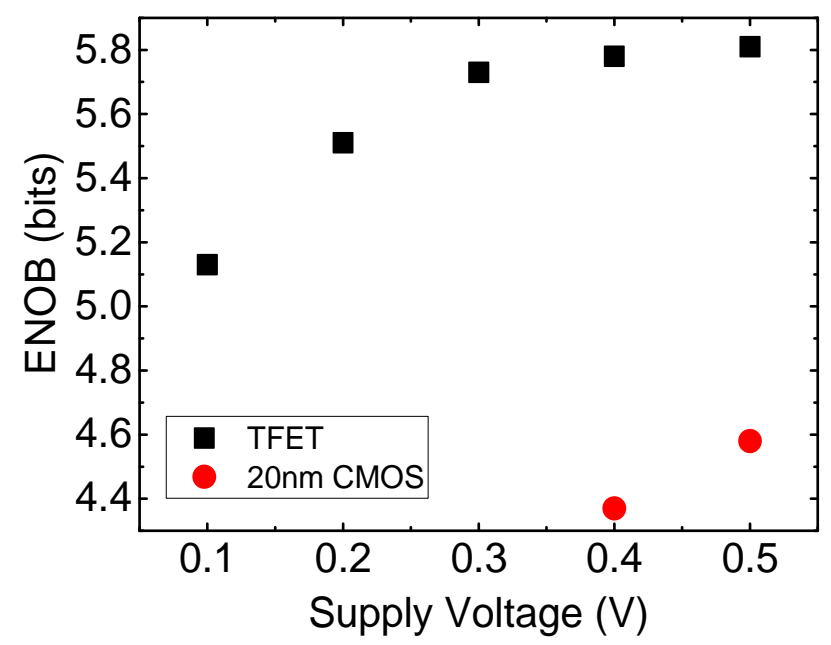

Figure 23. Simulated effective number of bits of SAR ADCs versus supply voltage.

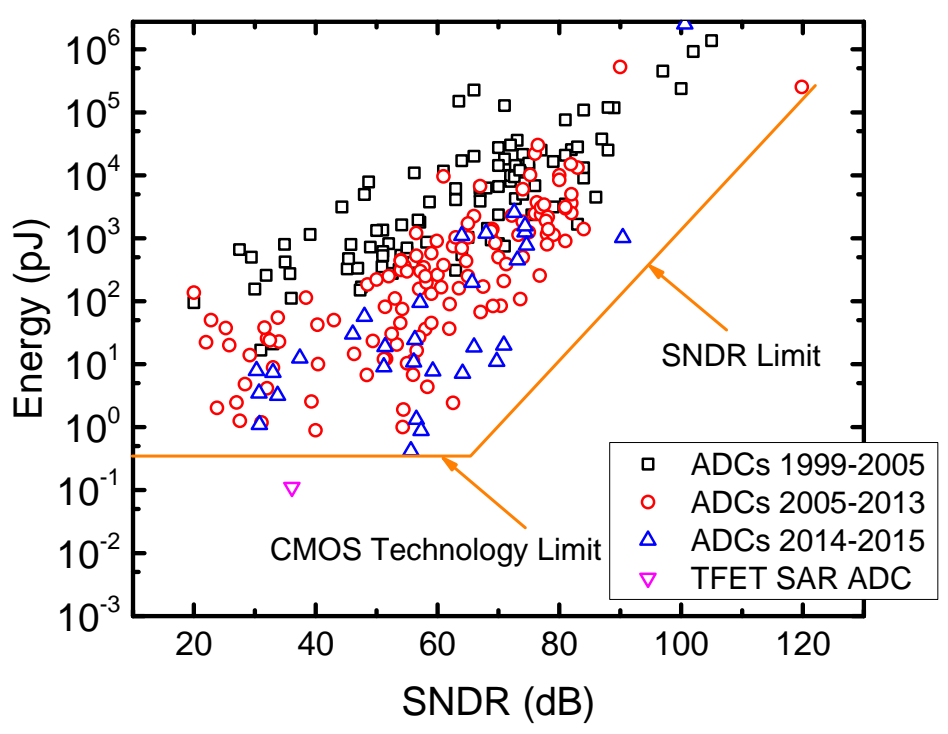

Figure 24. Energy versus signal noise dynamic range (note that the TFET SAR ADC is based on simulation results).

\subsection{Noise Shaping Low-Power $\triangle \Sigma$ SAR ADC Using TFETs}

TEFT-based NS $\Delta \Sigma$ SAR ADC is designed and evaluated using Cadence Spectre ${ }^{\circledR}$ with the transient noise simulation module. Figure 25 shows the schematic of the dynamic comparator using TFETs. The minimum TFET transistor length is $20 \mathrm{~nm}$. The supply voltage is $0.3 \mathrm{~V}$ to exploit the benefit 
of near-threshold operation. The temperature is at $25^{\circ} \mathrm{C}$. Under the normal condition, the external clock frequency is $25 \mathrm{MHz}$.

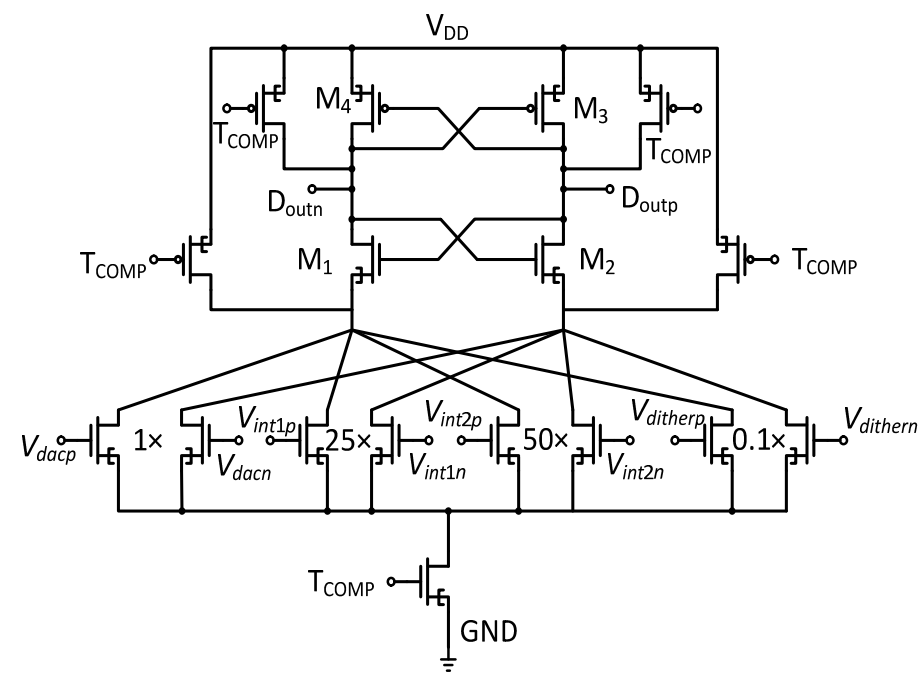

Figure 25. The comparator circuit used in the NS $\Delta \Sigma$ SAR ADC.

Figure 26 shows the output PSD of the NS SAR ADC when the input frequency is (a) $5 \mathrm{kHz}$ and (b) $25 \mathrm{kHz}$. The simulated Signal to Noise and Distortion Ratio (SNDR) for the $5 \mathrm{kHz}$ input signal is $72.14 \mathrm{~dB}$ and its SFDR is $76 \mathrm{~dB}$. Consequently, the ENOB for the $5 \mathrm{kHz}$ input signal is 11.69 bits. The harmonics of the $25 \mathrm{kHz}$ input fall out of Nyquist frequency and submerges in the shaped noise. The SNDR for the $25 \mathrm{kHz}$ input is $71.51 \mathrm{~dB}$, and the ENOB is 11.58 bits. The power consumption break-down is displayed in Figure 27a [28]. Energy and SNDR consumption of the current design are compared with various ADC data reported in the literature [24] is shown in Figure 27b. At a given SNDR, the TFET-based $\triangle \Sigma$ SAR ADC shows the best energy performance. For example, the 2nd order $\triangle \Sigma$ SAR ADC we designed (marked as a star in Figure 27b) exhibits the lowest power dissipation of the previously reported ADCs, with an SNDR greater than $62 \mathrm{~dB}$ (equivalent to resolution great higher than 10 bits).

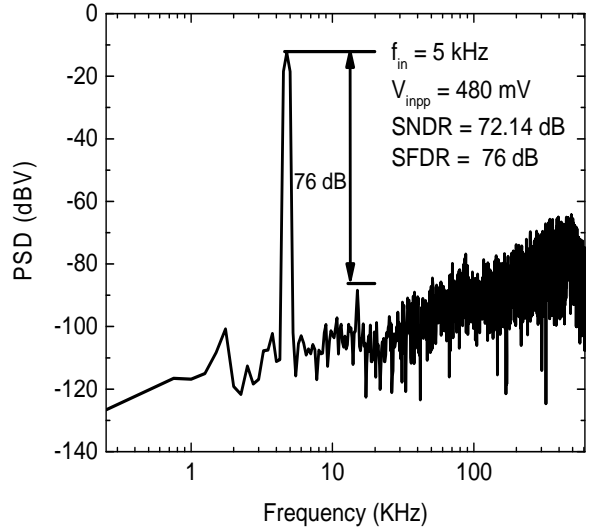

(a)

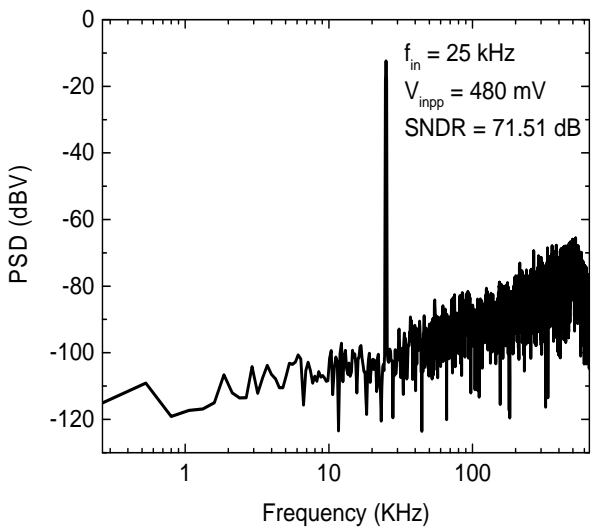

(b)

Figure 26. Power spectrum density versus frequency at (a) $5 \mathrm{kHz}$; (b) $25 \mathrm{kHz}$. 


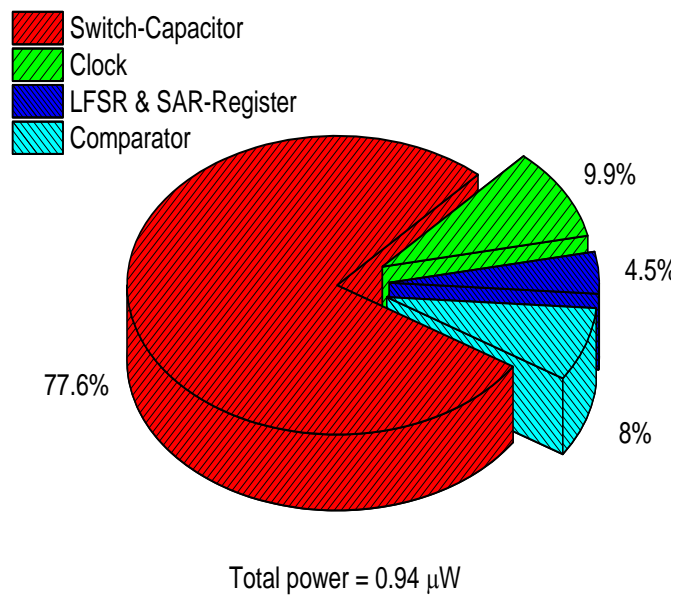

(a)

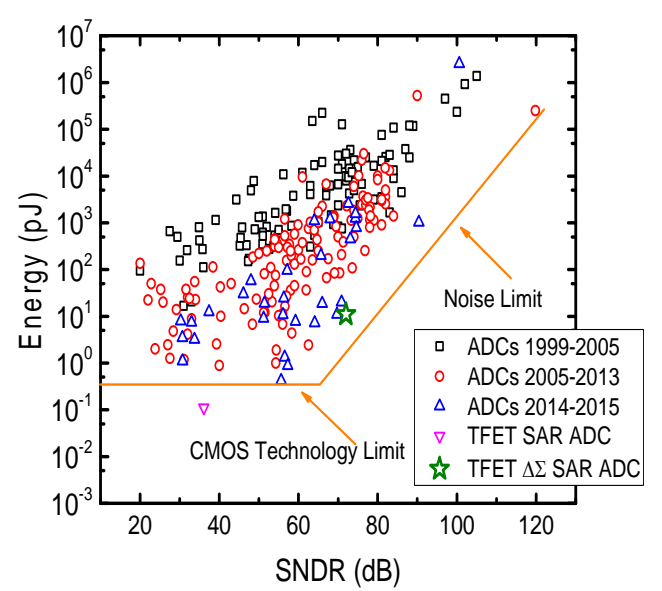

(b)

Figure 27. (a) Power distribution diagram; (b) Energy versus signal noise dynamic range.

\subsection{Bio-Inspired Ultra-Low-Power Computing}

The human brain is the most efficient low-power machine. A human brain contains about $10^{11}$ neurons and $10^{15}$ synapses to perform remarkable visual or other sensory perception tasks such as classification, recognition, and cognitive reasoning. It handles immense amount of data for real-time processing and consumes approximately $20 \mathrm{~W}$ of power. Traditional von Neumann computing systems based on CMOS technologies cannot achieve this level of energy efficiency. Neuromorphic hardware systems that potentially provide the capabilities of biological perception and information processing have gained much attention [75,76]. Bio-inspired neuromorphic computing may open a door to novel computation and communication paradigms. Figure 28 shows connectivity of biological neurons and synapses for signal transmission in a neural network.

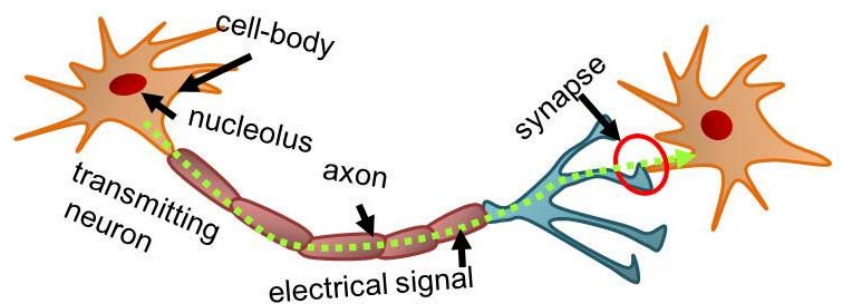

Figure 28. Schematic of biological neurons with synapses in a neural network.

Bio-inspired computing may be used as the next-generation ultra-low-power solution. A neuron receives information from many synapses and adds the information together with different weights, as represented in Figure 29a. When the summing signal reaches a firing threshold voltage in the membrane, it produces an output spike. An integrate-and-fire (IF) neuron circuit schematic is show in Figure 29b. Spiking neural networks (SNNs) are a prime candidate for enabling on-chip intelligence. Driven by brain-like asynchronous event-based computations, SNNs focus their computational effort on currently active parts of the network, thereby achieving orders of lesser power consumption compared to their artificial neural network (ANN) counterparts. 


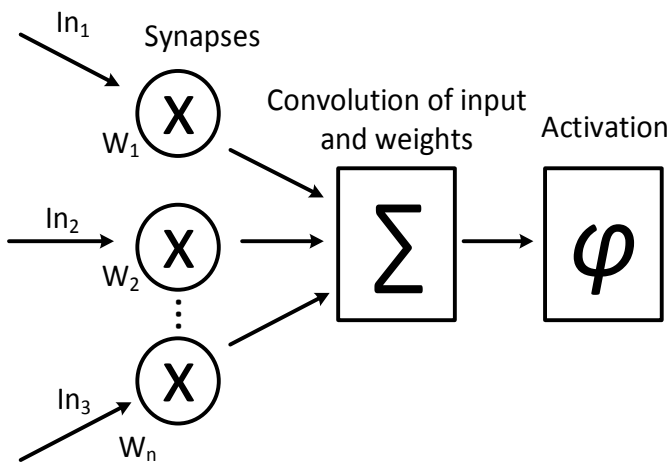

(a)

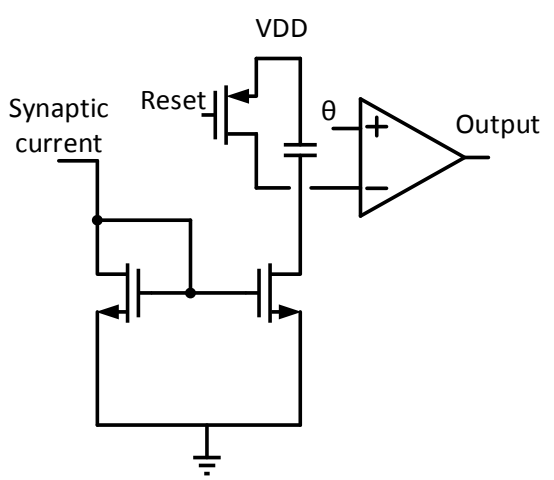

(b)

Figure 29. (a) Model of neuron summation; (b) An integrate-and-fire neuron.

IBM Research in 2014 demonstrated a large-scale digital CMOS neurosynaptic chip, named TrueNorth [77], with more than $1 \times 10^{6}$ integrate-and-fire spiking neurons and $256 \times 10^{6}$ synapses. TrueNorth, however, does not incorporate any information pertaining to the learning mechanisms. Neuron scientists discovered that learning rules follows spike-timing dependent plasticity (STDP) [78]. Brain processes asynchronously spike streams for recognition and extraction of repetitive patterns in a fully unsupervised way. In STDP unsupervised learning, the synaptic weights can be adjusted. The weight is increased if the timing difference between the post-synaptic pulse and pre-synaptic spike is positive, as shown in Figure 30. The weight is decreased if the timing difference between the post-synaptic spike and pre-synaptic spike is negative. This mimics brain learning capability. In addition, biological spiking neurons and synapses exhibit inherent stochastic nature. Noisy signals can also be processed with certain accuracy.
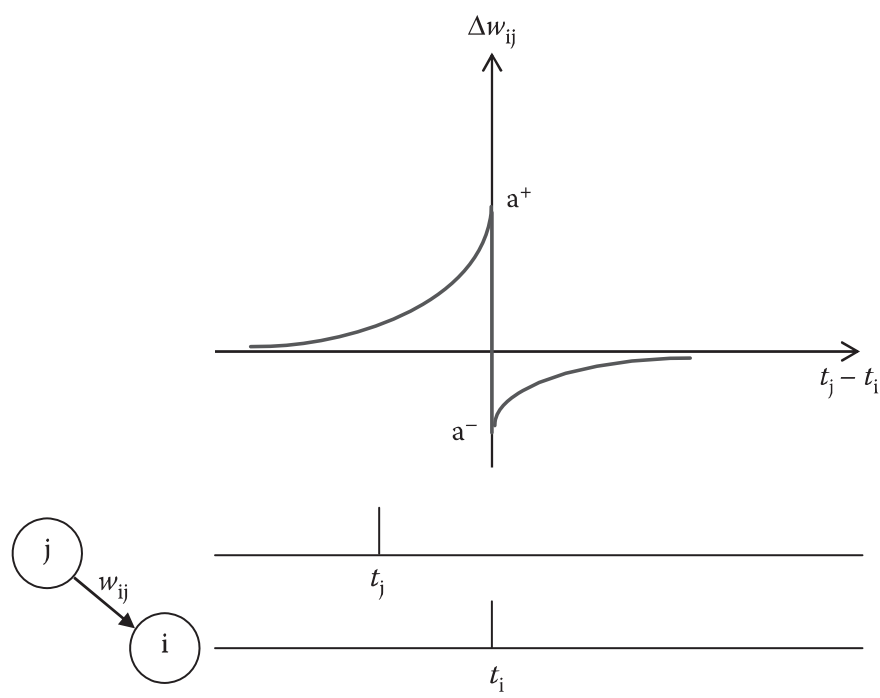

Figure 30. Pre-synaptic spike and post-synaptic spike for spike-timing dependent plasticity (STDP) learning.

Emerging nonvolatile resistive memory, phase change memory, and conductive-bridge memory are good candidates for the emulation of a bio-inspired system with binary synapses and stochastic STDP learning rules. Stochasticity is an inherent feature within the memristor. It causes the switching times from one state to the other to become variable based on the supplied input voltage and duration of the pulse. For example, applying a smaller voltage pulse but for a longer period of time also triggers the switching event. The memristor is a two-terminal device whose resistance is a function of its 
current state and input bias. It varies between a lower resistive state of $\mathrm{R}_{\mathrm{ON}}$ and a higher resistive state of $\mathrm{R}_{\mathrm{OFF}}$ similar to the RRAM performance described in Section 3.2.1. Innate variability of the memristor switching between its two states is embraced to model stochastic binary synapses. A simple threshold model incorporating the hysteresis output dynamics of the memristor with the added stochasticity and variable threshold is described as [79]

$$
d V_{T}=\alpha \theta\left(V_{T 0}-V_{T}\right) d t+\left(|V|-\Delta V-V_{T 0}\right) d N(\tau)
$$

where $V_{T}$ corresponds to the instantaneous threshold voltage calculated at every instant of time, and $V_{T 0}$ represents the switching threshold. That is, the point at which the switching of the device is almost instantaneous, and the probability is around 1. $\Delta V$ is an infinitesimal difference of the input value and the newly set threshold point. $\theta()$ corresponds to the step function, and $N(\tau)$ is the Poissonian process that adds the variability to the threshold. The resultant memristor output is an induced temporal switching stochasticity. The first term in Equation (4) is deterministic, and the second term in Equation (4) represents the stochastic behavior.

With the resistance change between two states, and the temporal variability in the switching behavior, the memristor is akin to a binary stochastic synapse. The use of a memristor within a crossbar structure provides an interconnected array in input and output neurons. The interactions between the pre-synaptic neurons and the post-synaptic neurons will impose levels of voltage across the memristors whose state will be updated in non-deterministic manner. Adding stochastic feature to the binary synapses makes them behave in a probabilistic manner in allowing the neuronal spikes to pass or induce a weak response as per the memristor state. This emulation of the noisy environment within the brain enhances the learning process for the neural network.

Figure 31 shows the input and output of an integrate-and-fire neuron with memristor synapses taking into account the stochastic behavior of the memristor.
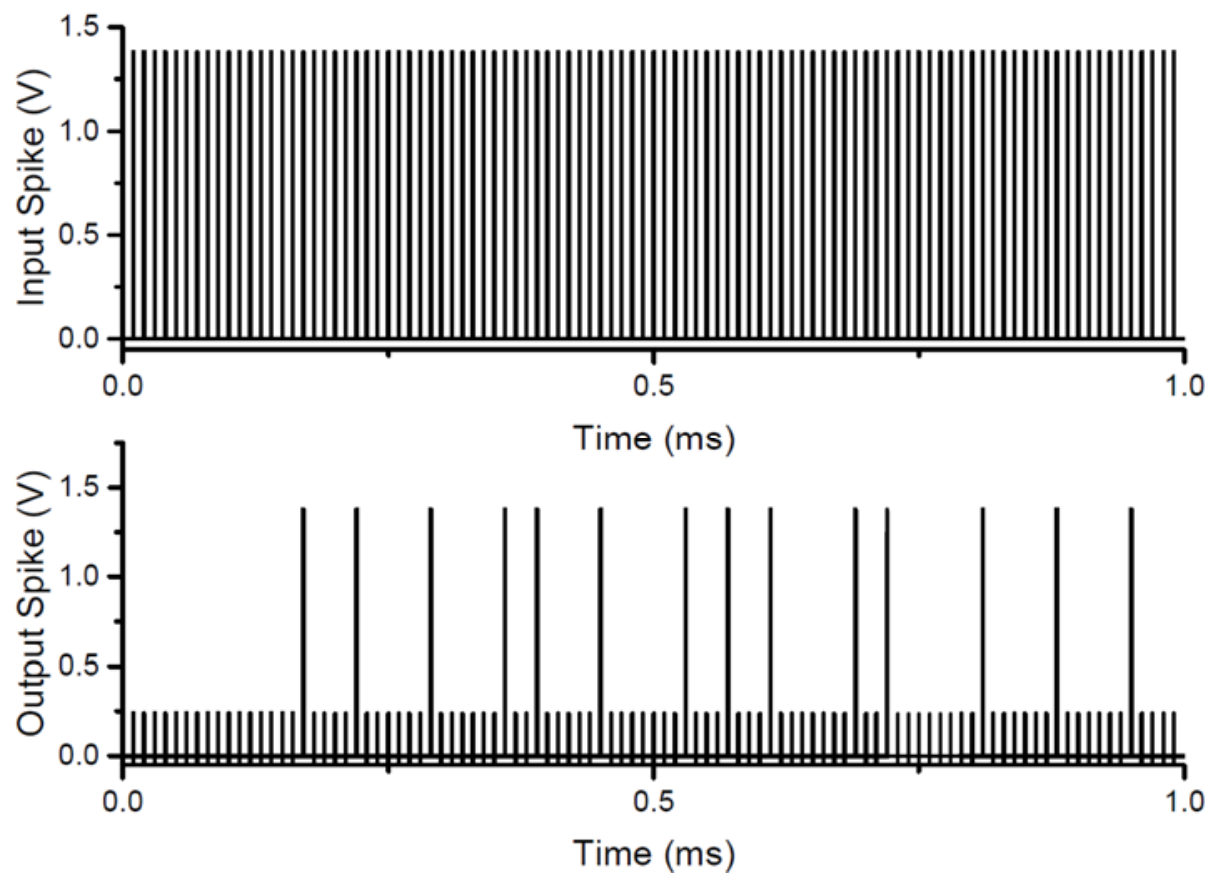

Figure 31. Input and output spikes of an integrate-and-fire (IF) neuron with memresitor synapses.

Recently, a heterostructure composed of a MTJ and a heavy metal as a stochastic binary synapse was proposed [80]. Synaptic plasticity was achieved by the stochastic switching of the MTJ conductance states, based on the temporal correlation between the spiking activities of the interconnecting neurons. 
The efficacy of the proposed synaptic configurations and the stochastic learning algorithm on an SNN trained to classify handwritten digits from a MNIST dataset was demonstrated. The power efficiency of the proposed neuromorphic system stems from the ultra-low programming energy of the spintronic synapses.

\section{Hardware Security}

IoT connectivity with embedded sensors, processors, and actuators that sense and interact with the physical world at any time and any place creates security and privacy challenges. IoT devices are venerable to hacking. For example, the Google Nest thermostat used in a smart home can be hacked by accessing the sys_boot pin in the Nest Thermostat [81]. The processing unit will start operating based on the incoming instructions from either the USB or the UART3 port once sys_boot is withdrawn significantly. The adversary might exploit this boot vulnerability to insert his or her own codes into the device. A vulnerable IoT device could be used to attack other components or devices that are on the same IoT network. The goal of such attacks is to leak private or unauthorized data for end-users though using backdoor insertion.

\subsection{Encryption}

Encryption is defined as one of the most widespread techniques that is utilized to protect the transceiving data from unauthorized users, snooping attacks. Several encryption methodologies have been proposed, but the more robust one is the Advanced Encryption Standard (AES) [82]. Implementing AES on a chip is very important in the IoT system. However, the hardware implementation of an AES algorithm is more complex compared to other encryption algorithms. Moreover, many side channel attacks have been demonstrated to recover the secret key using the accelerated algorithm [83]. The complexity of AES could be mitigated though partitioning the algorithm into segments, such as Shift row, S-box, and Mix column. For example, implementing AES encryption with 128 bit plaintext $(4 \times 4$ array, namely state machine $(\mathrm{SM}))$ can mainly be achieved in four steps, where the number of required rounds depends on the length of the encrypted key-bits. Each AES round includes four operations: SubBytes, ShiftRows, MixColimns, and AddRoundKey. SubBytes: Each incoming 16 bytes converts to a different value though a simple substitution operation using an S-box function, where a table with 256 values are introduced for substitution purposes. ShiftRows: This operation performs on each row of the state array, in which each row is rotated to the left via a specific number of bytes. This step is used to scramble the 128-bit data block. MixColimns: This operation is used to create a new column by multiplying each state array column by a matrix having 1, 2, and 3 numbers, where the new columns are exchanged with the one. The MixColimns transformation could be implemented using XOR with NAND logic gates (to perform shift and add operations). AddRoundKey: The last step is XORed the secret round key. Based on the aforementioned discussion, AES requires several XOR gates and shift operations, which could offer good advantages with certain technologies that provide low overhead on implementing XOR and shift operations.

Rivest, Shamir, and Adleman (RSA) [84] introduced a cryptographic algorithm for improved security. RSA is a public-key cryptosystem. The encryption and decryption operations of an RSA algorithm are achieved using two different keys, namely a public key and a private key, where the public key is used to encrypt the plaintext and the private key is utilized to recover (decrypt) the data at the receiver. The difficulty of implementing RSA cryptography is to produce the public and the private keys since these keys should be large prime numbers. Otherwise, it will be vulnerable to brute force attacks. Another kind of asymmetric key cryptography, called Elliptic Curve Cryptography (ECC), has been developed [85]. ECC provides good security with lower computation cost. ECC is suitable in many applications, such as healthcare systems, and wireless and mobile environments. ECC provides high-level security, which is similar to RSA cryptography, with a smaller key size. As a consequence, it will provide superior performance, cost less, and reduce power dissipation. Gura et al. [86] compared ECC and RSA performance using 8-bit microcontrollers. They were able to 
achieve a 1024-bit RSA private key operation with exponent $e=10^{16}+1$ in $0.43 \mathrm{~s}$ and 160-bit elliptic current point multiplication in $0.81 \mathrm{~s}$ with a clock speed of $8 \mathrm{MHz}$ on the 8 -bit microcontroller.

Even though AES and RSA encryption cryptographies can offer a high security level, they are not suitable for an application that requires a small area and low power dissipation, such as IoT systems. A lightweight encryption algorithm is more suitable for IoT applications since it requires a smaller area and lower power compared to AES and RSA encryption techniques. This is due to the fact that block size of the lightweight encryption cryptography is smaller than 64 bits, while the block size in AES is larger than 128 bits. For instance, both lightweight Data Encryption Standards (DES), DESXL and DESL, are proposed in [87]. The round function in DES can be replaced by S-box because a DES algorithm depends on the derivative data. This eliminates the need of the initial and final permutations. To further reduce the complexity of the encryption cryptography, two other encryption cryptographies, namely KATAN and KTANTAN, were introduced in 2009 [88]. KATAN/KTANTAN is a family of hardware-oriented block ciphers designed by Chrstophe de Canniere, Orr Dunkelman, and Miroslav Knezevic. The lightweight KATAN design consists of 256 rounds, shift registers, and nonlinear feedback functions. Each cipher has three different block sizes, 32 bits, 48 bits, and 64 bits, with 80 bit symmetric key size. The block of the KATAN cipher iterates for 256 rounds to produce the encrypted output data (ciphertext), where the key schedule with an 80 bit key size is shared with all KATAN blocks. Since the difference among the three cipher blocks regarding the required hardware resources is only the size of the register, we concentrate on the 32 bit blocks of the KATAN cipher. The 32-bit blocks is organized in 32 registers. The first 13 registers are located in the L1 part and the remaining 19 registers are in the L2 part. L1 and L2 blocks operate as a linear feedback shift register (LFSR). At each clock cycle, the data in both L1 and L2 blocks are shifted. L1 and L2 are used in both the encryption and decryption sides. For the encryption purposes, the plaintext is stored in both L1 and L2 blocks, where L1 carries the first 19 bits and L1 carries the remaining 13 bits of the plaintext. The computation of the two nonlinear functions, called $\mathrm{fa}(\mathrm{L} 1)$ and $\mathrm{fb}(\mathrm{L} 2)$, which consist of several XOR and AND operations, is achieved on data coming from the non-linear irregular factor (IR), different locations in L1 (at fb) and L2 (fa), and different key-bits, namely Ka and Kb.

Figure 32 shows both the least significant bits (LSBs) and the most significant bits (MSBs) for each L1 and L2 registers. For each clock cycle, the data in both L1 and L2 are shifted. Ka and Kb keys with IR are produced from two other blocks at each round. Figure 33a demonstrates the IR block, which contains 8 bit LFSR. Two operations are done in this block: first, counting the number of the rounds, and generating the irregular new value for the two function ( $\mathrm{fa}$ and $\mathrm{fb}$ ). The encryption process is complete once the number of rounds reaches 254 cycles. Another important block of the key schedule is shown in Figure 33b. This register has 80 bit LFSR, where the value of the secret key is loaded to this block before the encryption is started. Each round key is generated by shifting one bit in the LFSR generator. The two keys (Ka and $\mathrm{Kb}$ ) are produced from the last two significant bits every two cycles. Equation (5) shows the reciprocal polynomial of the LFSR generator with 4 taps located at 13th, 50th, 60th, and 80th bits, which are chosen for the 80 bit shift register. The definition of the key, which is referred to $K$, and the buskey of round $j$ is presented in Equation (6).

$$
\begin{gathered}
f(x)=x^{80}+x^{61}+x^{50}+x^{13}+1 \\
k_{j}=\left\{\begin{array}{lr}
K_{j} & j=0 \ldots 79 \\
k_{j-80} \oplus k_{j-61} \oplus k_{j-50} \oplus k_{j-13} & j>79
\end{array}\right.
\end{gathered}
$$




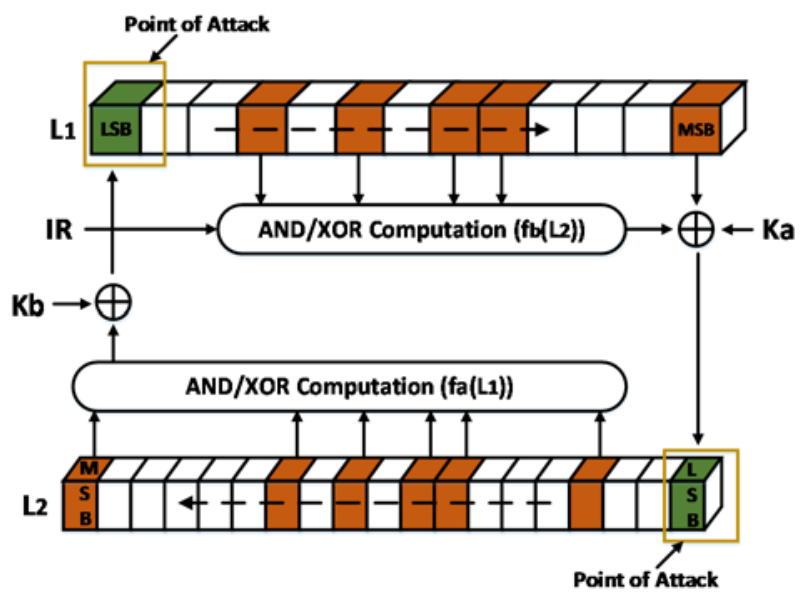

Figure 32. KATAN encryption scheme.

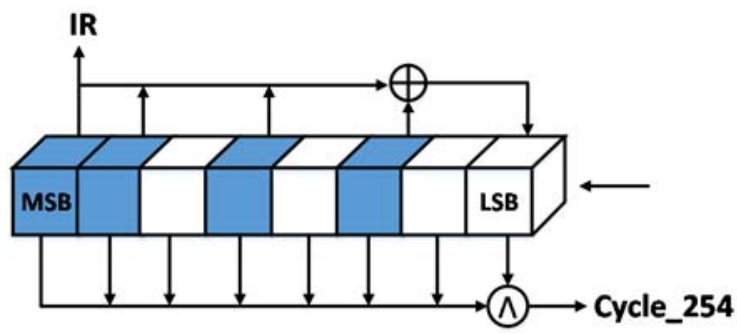

(a)

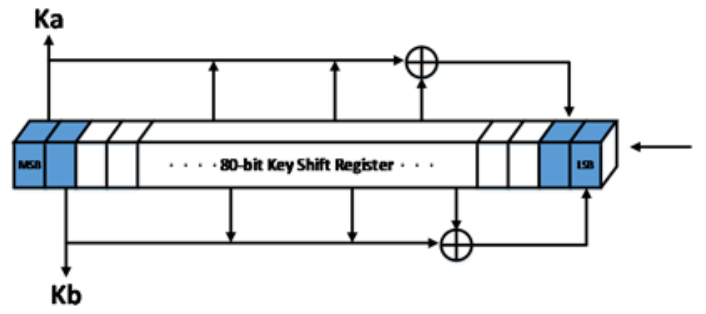

(b)

Figure 33. (a) Irregular factor (IR) block register; (b) generations of the two KATAN keys.

Equations (7) and (8) illustrate the two nonlinear functions ( $f a$ and $f b$ ) for KATAN cipher including the calculation of the two blocks (AND/XOR operations). We chose KATAN encryption with 32 bits. The locations of the bits in both $L 1$ and $L 2$ registers have been specified to achieve the computation in $\mathrm{Fa}$ and $\mathrm{Fb}$ functions, as shown in Figure 33. Note that the locations of these bits can be different if the block size of the KATAN cipher is changed.

$$
\begin{gathered}
f_{a}\left(L_{1}\right)=k_{a}+L_{1}[12]+L_{1}[7]+\left(L_{1}[8] \cdot L_{1}[5]\right)+\left(L_{1}[3] \cdot I R\right) \\
f_{b}\left(L_{2}\right)=k_{b}+L_{2}[18]+L_{2}[7]+\left(L_{2}[12] \cdot L_{2}[10]\right)+\left(L_{2}[8] \cdot L_{2}[3]\right)
\end{gathered}
$$

\subsection{Side Channel Analysis}

Side channel information analysis, specifically for power signature can be used to extract the digital key stored in a system. In an IoT world, the ubiquitous distribution of devices creates the possibility of accessing a device physically for performing side channel attack. Therefore, having a defense mechanism for this type of attack should be taken into account in a system design, but in addition to considering the power budget of the system. Researchers have worked for a while to counter a known and common side channel attack named, differential power analysis (DPA) [89,90]. Accordingly, the defense techniques (or cryptographic systems) can be realized at hardware-level and software-level (or algorithmic-level). These systems should be designed with specific functionalities that can block at least a certain and sufficient information leakage. As an example, multiple keys can be generated using a hashing algorithm that makes it difficult to fully execute an attack. Another technique suggests using masking methods (which means using additional mathematical functions) for the non-linear part of encryption algorithm [91] to further improve the security level. Additionally, the system voltage and frequency can be randomly varied to randomize the behavior of time and power 
traces, so as to prevent side-channel attacks at the gate-level. Yang et al. [92] proposed the employment of sense amplifier-based logic style for cryptographic algorithm implementations that makes power consumption independent (or irrelevant) of the processed data. Similarly, a traditional circuit level protection scheme is current mode logic (CML), a traditional circuit level protection scheme that provides both power efficiency and security enhancement. In order to evaluate a system's security, we cannot solely focus on the differential power analysis; other attacking schemes such as correlation power analysis should be considered.

Differential power analysis and correlation power analysis will now be discussed. Performing correlation power analysis on the KATAN cryptographic system [93] has been studied. According to [94], the intermediate values in computations of a cryptographic system during differential power analysis must be extracted and identified. These values along with the plaintext and ciphertext help to discover the keys. A smaller size of round keys (or intermediate keys) results in fewer computations of the DPA and consequently an easier system key analysis and discovery. Besides acquiring the actual power traces from the system, a number of key guesses are used to calculate the intermediate values that are considered hypothetical power traces. Next, the actual and hypothetical power traces are classified by a selection function, and analysis of the function outcome reveals a peak for the correct key hypothesis. An extension of the DPA in which a power model is used along with the intermediate values for computation of the hypothetical power traces is called correlation power analysis (CPA). The actual power and the predicted power traces are input into a correlation function to find the highest correlation value that is perhaps corresponding to the correctly guessed key. The leveraged power model in the CPA is the Hamming weight model; in the DPA, it is Hamming distance model.

The authors of [93] proposed a security evaluation of the KATAN family of cryptographic systems by analyzing the algebraic and the cube attacks. Additionally, the possibility of attacking a KATAN system by side channel analysis was mentioned. According to the KATAN algorithm, the plaintext and the ciphertext are related to the intermediate keys through two nonlinear functions that are "fa" and " $\mathrm{fb}^{\mathrm{b}}$. Next, the output bits of these two functions are the intermediate values or the targeting points of the attack. These two points can be seen in Figure 32. The hardware implementation of the KATAN cryptographic algorithm mainly consists of D flip flops. Thus, the overall power consumption of the system is largely dependent on these elements. As a consequence, an attack model that maximizes the contributions of the nonlinear functions to the system power traces must be utilized. The maximization can occur (in static logic style) by constructing the plaintext based on the convention of having a logical one-to-zero or zero-to-one transition at the one-function output bits for certain clock cycles, which causes a closer relationship between the power traces and the key. In this way, each portion of the key is revealed in every clock cycle until the whole key is extracted.

\subsection{Supply Chain Security}

Protecting electronic circuits and systems from counterfeiting IC in the supply chain is a concern. In general, attackers usually use cheap and simple methodologies in order to counterfeit or illegally copy chips. The produced chips might be unreliable and not work properly due to counterfeiting. Such counterfeited ICs may fail the system and consequently could put human beings' life in danger. The program of the Supply Chain Integrity for Electronics Defense (SHIELD) has been supported by the Defense Advanced Research Projects Agency (DARPA) in the United States to prevent counterfeiting and protect ICs via increasing the complexity of the design, which leads to a significant increase in the cost. In this case, the packaging of ICs consists of an encryption technique, e.g., National Security Agency (NSA) encryption, near-field communications, and sensors [95]. The occupied area for the trustworthy hardware will be approximately $100 \times 100 \mu^{2}$ (dielet), which is important for prohibiting attackers from accessing or reverse-engineering the dielet. ICs can be authenticated by using physical devices, called external probes, which will give an inductive/RF near-field reader that powers the dielet for a period long enough to exchange information that allows the dielet to identify and authenticate itself and provide an update of its passive environmental sensor readings. 
The SHIELD program provides a proactive and comprehensive solution that eliminates all pervasive forms of counterfeiting. The secure tracking of packaged electronic components enhanced by a strong root of trust and a reliable communications and power link will be a critical asset in terms of securing electronic systems both in military and commercial platforms.

The hardware-based threats are essentially categorized into three domains: hardware Trojan injection, IP piracy/IC overbuilding, and reverse-engineering. Adversaries in untrusted companies or design houses may be able to inject malicious circuits, namely hardware Trojans, into the original IP design. Moreover, a malicious insider might copy the chips without the permission of the designer and overbuild the IC chips for their own profits. An IP could also be reverse-engineered and overbuilt via an attacker. The vulnerability of chip security during manufacturing has spurred research on countermeasure methods. One of them is the logic encryption technique. Figure 34 presents the IC design flow combined with the logic encryption technique. Instead of shipping the original netlist to the offshore manufacturing foundry, a logic-gate level encryption technique is applied to protect the IP design at low cost. After retrieving the fabricated chips, in order to recover the correct outputs of the design, the correct key-bits should be provided to the encrypted circuit, for certified IP owners to unlock the chips. However, upon employing the invalid key-bits, the locked circuit should show the incorrect outputs.

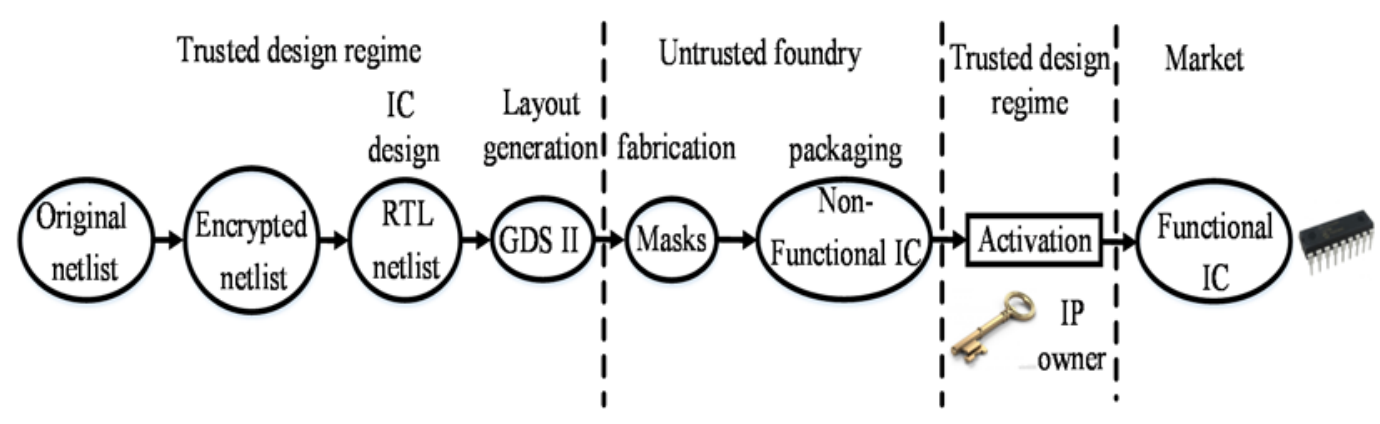

Figure 34. Supply chain security.

\subsection{Logic Locking}

Logic locking (or logic obfuscation) prevents IC piracy and overproduction attacks from exposing the correct functionality of an IC via inserting additional gates with key-bits. In combination encryption, many methods have been proposed such as random insertion, fault impact analysis, and logic obfuscation. In [96], Rajendran presented a fault impact analysis (FA) method to increase the security level of the random logic encryption. In the FA approach, the new gates are inserted based on the stuck-at fault model. First, the fault impact for each gate is calculated by computing the stuck at zero and at one. Afterwards, for each iteration, a new gate can be inserted at the highest fault impact on the output until the Hamming distance becomes $50 \%$ (or close to $50 \%$ ) or until all of the supplied 128 key bits are finished. For robust logic obfuscation, the key-related gate-bits are injected in a certain way into the design, which makes the key information extraction process difficult to achieve [97]. Yasin et al. improved on the work by inserting more pairwise keys [98]. In [99], IC protection is performed by insertion of process variation sensors inside the design at specific selected nodes along with the generation of a unique key for each IC. The maximum achieved HD from this technique was around $18 \%$.

Alasad et al. [100] demonstrates a secure circuit design by leveraging multiplexers as key gates. To maximize the protection of an IC from various attackers, the insertion of Multiplexer (MUX) at each output bit, as shown in Figure 35, is proposed. The original output bit and its complementary will be fed into a two-input MUX, along with a key bit for the selection of each MUX. The values of the key bit selection must be random with half zeros and half ones to produce 50\% HD. Since each output bit and its complementary are connected to a MUX with a random key bit selection, each output bit of 
the IC is changeable once the key is changed. In this case, not only is the HD between the corrected and corrupted outputs around $50 \%$, but the value of each output bit is also variable. An assailant cannot figure out the functionality of the design because each output bit will vary according to the supplied key via the LFSR generator, which is used to generate random keys (each key is generated to have randomly half zeros and half ones, as mentioned). Since the key value is unpredictable due to the random generation, each output bit will be consequently arbitrary. Once the correct user key is inserted, the output of the payload will be set, and the enable (EN) of the LFSR generator will then be disabled, while the activation signal (A) will be activated to initialize the values of the MUX selections. Then, the functionality of the circuit will be correct. If the value of one bit in the user key is incorrect, the corrupted output ratio will still be around 50\%. Although inserting MUX at each output bit will obviously maximize the protection of the design, as well as the ambiguity of an attacker, the power and area overheads will largely increase. Therefore, this technique is more suitable either for large circuits that include a large amount of output bits or for an expensive IC chip. In both half and full MUX insertions, if there is an inverter at an output, we replace it with an MUX by switching its inputs. Furthermore, all components of the encrypted circuit (in half and full MUX insertion techniques) are made at a pre-layout stage.

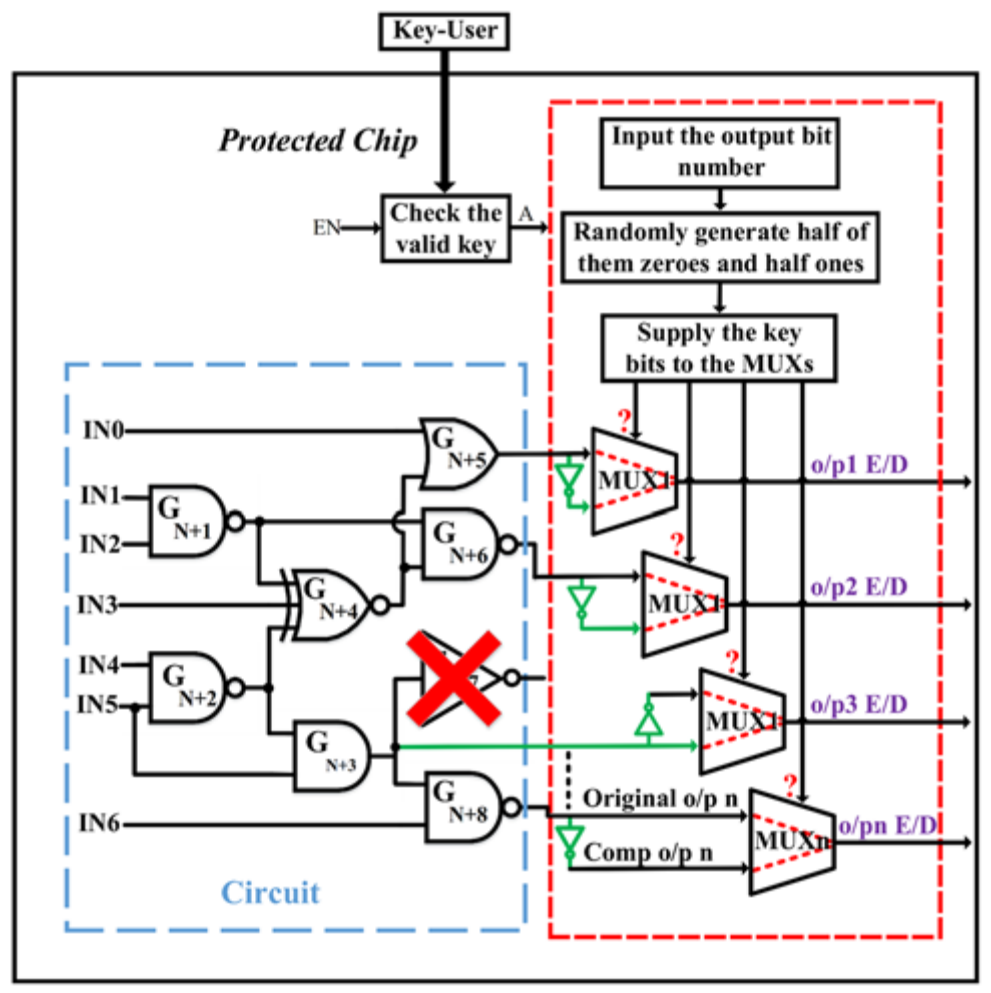

Figure 35. Logic encryption based on full Multiplexer (MUX) insertions.

Figure 36 demonstrates the analyzed HD for the combinational (ISCAS'85) and the sequential (ISCAS'89) benchmark circuits based on the full MUX insertions for logic encryption, where the minimum required length of LFSR to achieve the HD should be the same as the number of primary output-bits. The achieved HD for these benchmark circuits is 50\%, except for S9234, which is $48.72 \%$ due to its having an odd output number. 


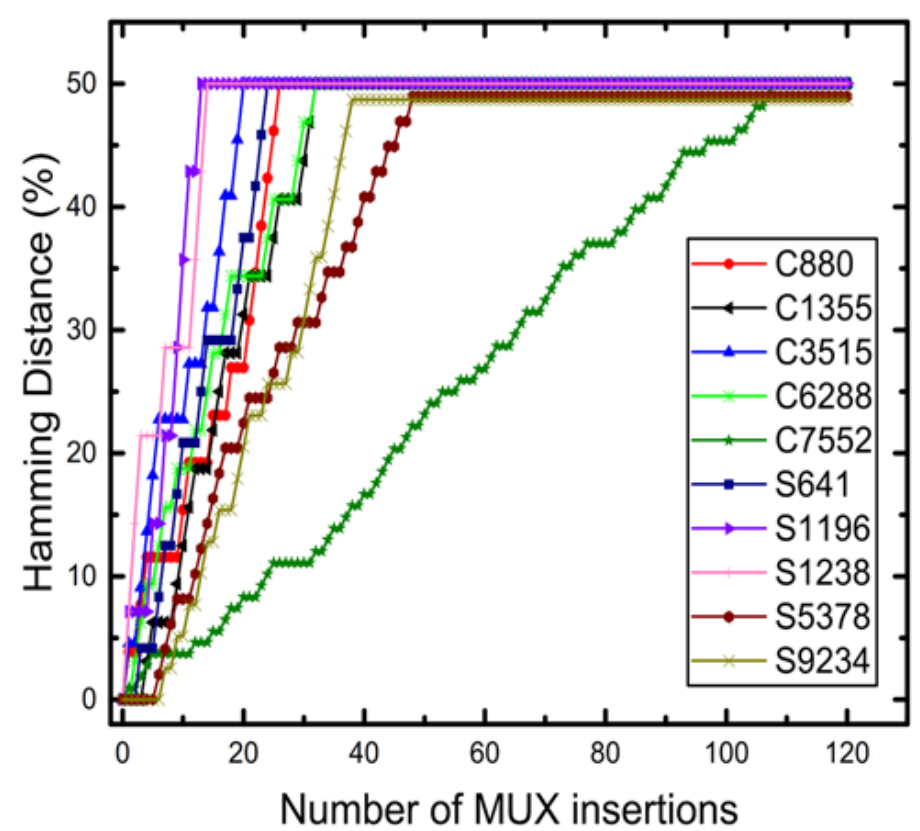

Figure 36. MUX insertions based on the full output number for different ISCAS '85 and '89 benchmark circuits.

The delay, power, and area overhead for each benchmark circuit is measured using the design compiler tools from Synopsys with a $45 \mathrm{~nm}$ CMOS library. Since MUXs were inserted only at the output of the netlist, the delay overhead (timing path) is almost zero for all of the benchmark circuits. Meanwhile, the power and area overheads for each benchmark circuit depends on the number of output bits. Figures 37 and 38 show the power-delay and area overheads. On average, half MUX insertions save more than $3.6 \times$ area overhead and $3.4 \times$ power-delay overhead compared to those of fault impact analysis, while full MUX insertions require less than half of the area overhead and half of the power-delay overhead that the fault impact analysis needs.

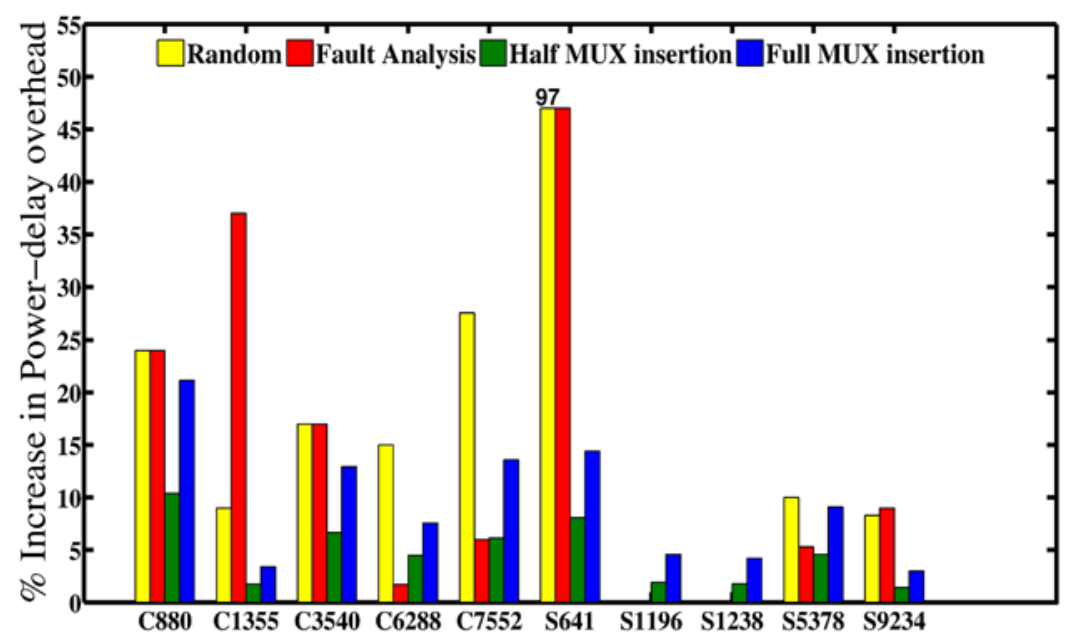

Figure 37. Comparing the power-delay overhead of random, fault analysis, and full/half MUX insertions for logic encryption. 


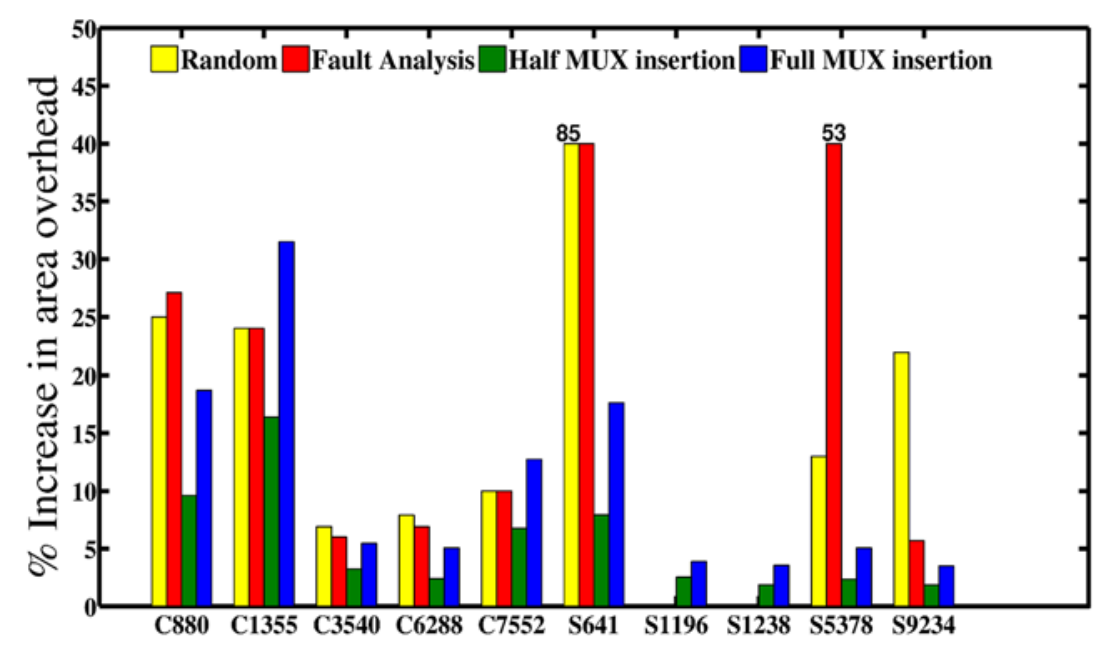

Figure 38. Comparing the area overhead of random, fault analysis, and full/half MUX insertions for logic encryption.

Several kinds of attacks have been proposed to reveal the vulnerabilities of various logic locking methods to dispute the correct key of the locked circuit [101]. However, the most powerful one is a Boolean satisfiability (SAT)-based attack [102]. By employing few discriminating input patterns, an SAT-attack successfully exposes the secret key of all logic locking methodologies. These discriminating input patterns are supplied to the encrypted circuit and their corresponding outputs are compared with the correct output patterns, where they are obtained from an activated IC in the open market. An SAT algorithm is used to determine these input-output golden pairs. As a result, an SAT attack uses only the affected input patterns and therefore decrypts a large-scale circuit that has large key sizes within a few minutes.

An SAT attack can be mitigated via incorporating a small logic circuit as a Tree of AND gates that works as a one-function output. Yasin et al. [103] implemented a lightweight logic block, namely the Anti-SAT technique, to protect the locked netlist from an SAT-based attack. Part of the input key-bits (KA) is used for encrypting and decrypting of the locked design, while the rest of the key-bits (KB) are utilized to thwart the SAT solver. The number of iterations that the SAT attack needs to extract the secret key increases exponentially with the number of the Anti-SAT key-bits (KB). Even though the Anti-SAT block successfully prevents an SAT attack when KB is larger than 64 bits, this technique is valuable in tracking a signal-based attack, called signal probability skew (SPS) [104]. SPS can easily identify and remove the incorporated Anti-SAT circuit within a few seconds since the two outputs of the two Anti-SAT complementary blocks should have the highest differential signal probabilities. The SPS-based attack removes Anti-SAT from all encrypted netlists in less than 2 min for a large-scale circuit.

\subsection{Logic Locking Using All-Spin Logic Device (ASLD)}

The ASLD can naturally perform as a majority gate (MG) operation. The principle of the MG is that the value of the primary output relies on the values of the majority inputs. Based on this phenomena, the ASLD can implement any logic gate. For instance, a designer can easily obtain an $\mathrm{N}$-inputs NOR gate by making the value of the fixed magnet as ' 1 '. By changing the magnetization direction of the fixed magnet (making the value of the fixed magnet as ' 0 '), the design can perform as an N-inputs NAND gate. To obtain AND and OR gates, one more magnet layer must be added at the primary output. Based on this analysis, an ASL device is considered a polymorphic gate by employing its unique feature. The device gives us an opportunity to change the functionality of the circuit with the same structure and without any extra hardware by making one of the primary input as an external key. As shown in Figure 39, the structure of ASL can provide four different gates with the same circuit: 
AND, OR, NAND, and NOR using only 4 magnets. Where A and B are the primary inputs, Key and VDD are used to change the functionality of the circuit. We make the third input of magnet $(C)$ as an external key input. The circuit can be switched from an AND to an OR gate or from an OR to an AND gate by only exchanging the value of the key from ' 0 ' to ' 1 ' or from ' 1 ' to ' 0 ', respectively, when the VDD is positive. On applying a negative VDD, the design can work as a NAND or a NOR gate if the value of the key is ' 0 ' or ' 1 ', respectively. There is another way to get a NAND or a NOR gate. A designer can apply only a positive VDD and add one more magnet at the output of an AND or an OR gate, respectively.

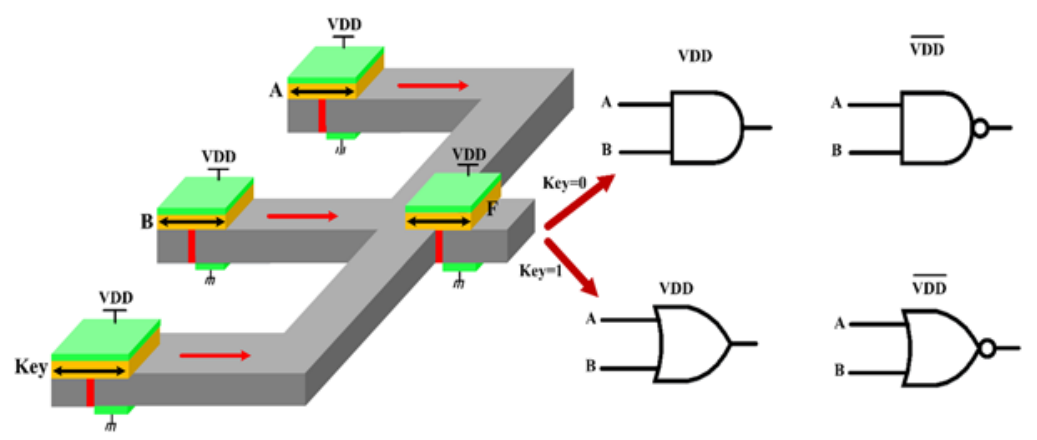

Figure 39. All-spin logic (ASL) AND, OR, NAND, and NOR polymorphic gates.

Similarly, XOR and XNOR gates can be built as shown in Figure 40.

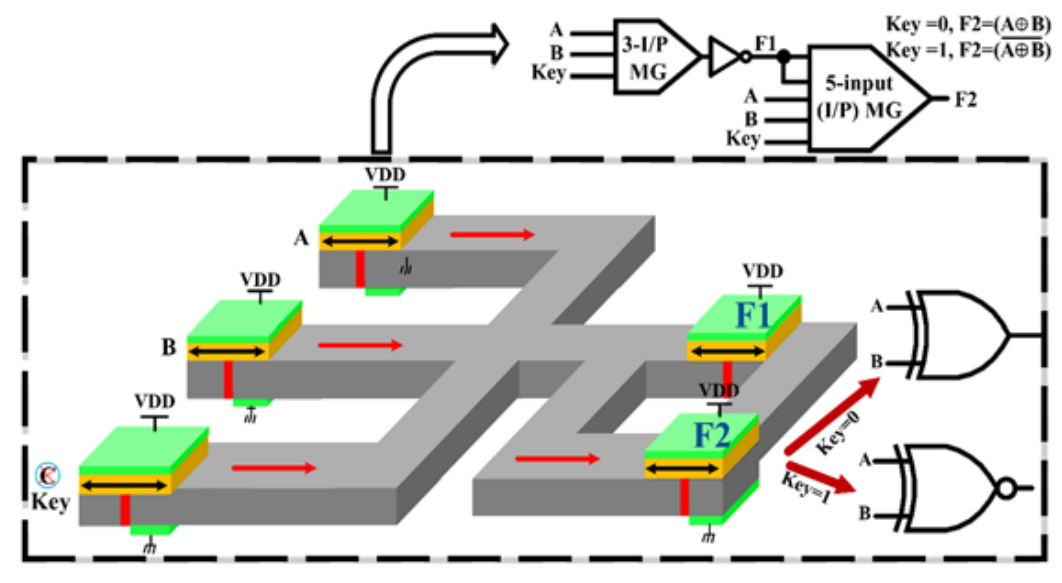

Figure 40. ASL XOR and XNOR polymorphic gates.

Using the ASL logic developed above, one can construct SAT-resilient design [105], as shown in Figure 41. In Figure 41, $\mathrm{X}$ is the distinguished input-bits, and K1, K2, and K3 are the external input keys. The final output of SAT-resilience can be either " 0 " or " 1 " (based on the designer's configuration) on applying the correct key, and the last inserted key-gate (between the original output of the encrypted circuit and SAT-resilient output (S-O/P)) must be either XOR or XNOR, respectively, in order to obtain the correct output. 


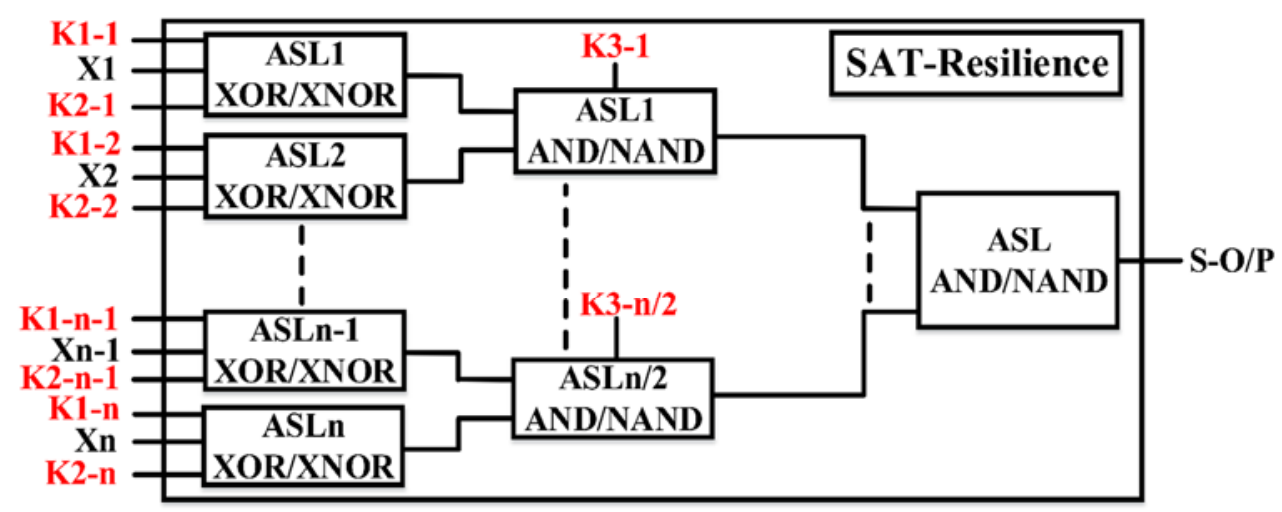

Figure 41. Scheme of satisfiability (SAT)-resilient design using ASL.

\subsection{Split Manufacturing}

Split manufacturing is a way to partition digital circuits into many parts for security purposes. The authors of [106] introduced a technique though supplying three-dimensional combination technology in split manufacturing. The authors implemented an algorithm to analyze the graph of a circuit and disconnect certain wires from the design to prevent an attacker from obtaining the correct design. Another proposal [107] was presented by Rajendran et al. whereby split manufacturing at layer-3 mental was examined. The benchmark circuits have been partitioned into many parts without any connections among them. Afterwards, they developed a fault analysis algorithm in order to switch the pins at layer 1 and 2 metals because the connections of the gates for any circuit are placed in the first and second layers, which might help an attacker in an untrusted foundry for getting the original design. The implementation of split manufacturing design before the second metal layer was proposed by Vaidyanathan et al. [108]. Therefore, only the information at the gate level of the circuit will be revealed to the untrusted companies. A similar method was achieved in detail for analog and digital IC circuits in [109]. The technique against recognition IC-based attacks has also been included, supported by experimental results, where an SRAM with a $1 \mathrm{~KB}$ size and a digital-to-analog converter with 14 bits have been used. Jagasivamani et al. [110] implemented many front end locking techniques and evaluated them based on security metrics and performance overhead, where statistical analysis tools have been utilized to perform these techniques in a large-scale system design. Split manufacturing methodologies could also be used to detect a malicious Trojan using only the test back end of line (BEOL). Leveraging split fabrication in a field programmable gate array (FPGA) chip was presented for asynchronously designed digital circuits [111]. A compression result between using the standard process and the split fabrication indicates that the standard process can outperform split manufacturing in terms of providing better performance with less power-delay product penalty.

Although RF design circuits are more vulnerable to IC piracy than other digital circuits, split manufacturing has not been suggested for protecting RF circuits from such serious attacks. Split manufacturing is better applied in RF circuits than in any other digital design due to their unique metal features. More specifically, both the direction of the wires and their length are functional parameters in the metal layers of RF circuits, while, in the digital circuit, the layers are extracted as net connections. In additional, the metal layers in RF circuits are not only utilized as interconnections between the modules and logic gates as in digital designs, but they are also used to build small parts of the chip functionality. For example, the capacitors and the indictors are leveraged to build the upper level and the top metal layers, respectively.

Split manufacturing is a good candidate for making RF designs that are more secure from IC piracy and other threats. Statistical analysis with experimental results are achieved for all kinds of RF components to emphasize the value of using split manufacturing for protecting RF circuit purposes. The benefits of removing the metal layers in RF designs are listed; (1) the connected nets among the parts of the designs are concealed, and this increases the ambiguity of attackers to identify the original 
design, and (2) the passive parts of the design that are implemented in the metal layers are abstracted. It is easy to retrieve the interconnection among the internal parts in an RF circuit since it has few components. Instead, using a split fabrication technique can help infer the missing passive parts in RF circuits. The main advantage of leveraging split manufacturing in the RF design is the difficulty an adversary would face retrieving the types and sizes of passive components. This emphasizes the importance of using such a method in RF circuits. The dilemma in RF designs regarding the routing, analyzing, and mapping of the components by an attacker is eliminated by using a split fabrication methodology. Moreover, the proposed recognition technique-based attacks [88] cannot successfully infer the original design of an RF circuit implemented using split manufacturing. Extra dummy components and wires could be added to the design using an obfuscation method to elevate the security of the chip. This will increase the difficulty of an attacker to recognize the number, size, and location of passive components.

Figure 42 shows the split fabrication of a Class AB RF circuit for power amplification at an untrusted foundry (Figure $42 \mathrm{a}$ ) and the completion of the fabrication at a trusted foundry (see Figure 42b).

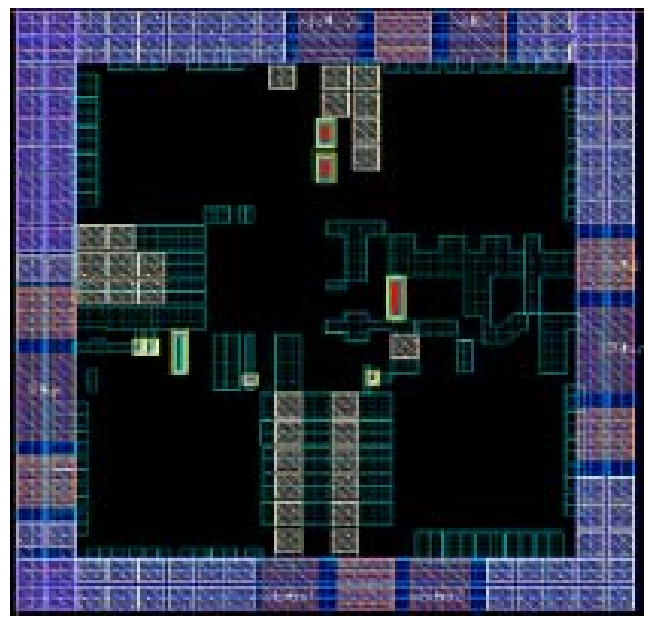

(a)

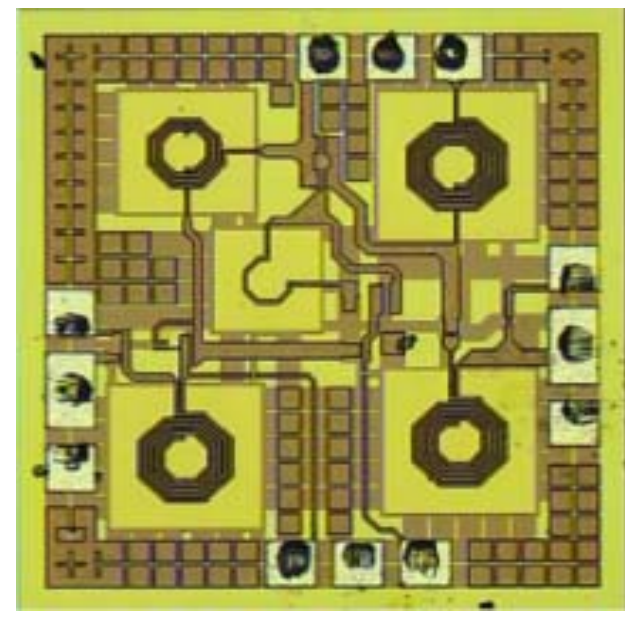

(b)

Figure 42. Split fabrication of a Class AB RF power amplification circuit (a) before metallization at a untrusted foundry and (b) after metallization at a trusted foundry.

The three-dimensional integration extends the design to the third dimension using several layers of through silicon vias (TSVs) interconnection (see Figure 43 for detail). In addition to increased chip density, TSVs reduce interconnection length and hence decrease power and delay. Three-dimensional integration also introduces security vulnerability opportunities. This includes side channel analysis attack prevention, trusted computing design, and the prohibition of supply-chain-based attacks [112]. For instance, the dimensions of an integrated circuit containing many dies from different sellers are not secure because not all of the IP providers follow a similar level of die certification. A more practical way is to use an interposer 2.5D method for integrating dies from Third-Party (3P) sellers/vendors. Therefore, securing inside dies is a main concern for the developed three-dimensional chips. In [113], the authors proposed a technique to obscure the vertical communication channel in the network on chip systems, which is useful for preventing reverse-engineering-based attacks and consequently making the system more secure. 


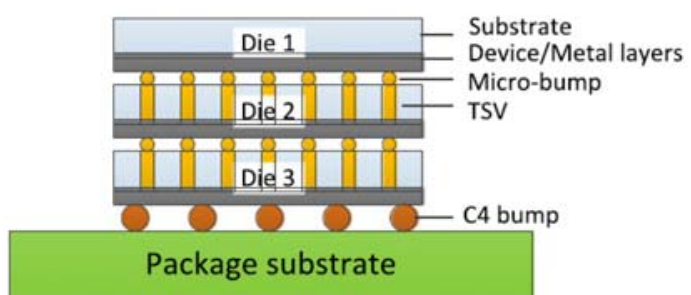

(a)

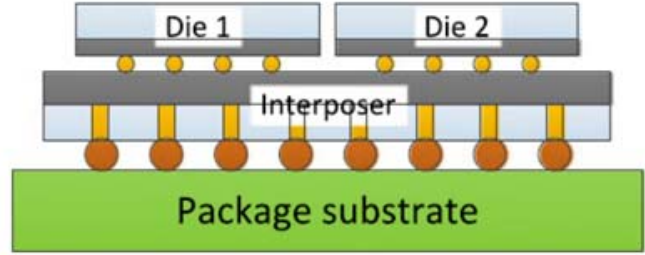

(b)

Figure 43. (a) Three-dimensional integration of multiple dies using through silicon vias (TSVs); (b) 2.5D integration of multiple dies using an interposer.

\section{Hardware Security Enhancement Using Emerging Technologies}

The unique characteristics of emerging devices can be used to accomplish a higher security level with lower performance penalty for ICs compared to CMOS technology if these features are employed properly. In general, emerging devices have been proposed since CMOS technology cannot be significantly scaled down. Furthermore, they can help improve the performance of the circuit and simplify the design structure for security applications, e.g., IC protection, hardware implementation of cryptography, and Trojan detection and prevention [114]. In this section, KATAN light-weight encryption using current-mode logic against correlation side-channel power analysis, logic locking, and camouflage layout using emerging SiNW technology are presented.

\subsection{KATAN Light-Weight Encrpytion Using TFET Current-Mode Logic for Low Power}

It is well known that the key idea of differential power analysis is based on the power consumption during circuit transition. In static CMOS logic, major power consumption occurs when the output of logic undergoes a $0 \rightarrow 1$ (or $1 \rightarrow 0$ ) transition. Because of this symbolic characteristic of static logic, the genuine cryptographic algorithm is vulnerable to the DPA attack. On the contrary, the common-mode logic (CML) structure is naturally resistant to a DPA attack considering the relatively constant power consumption for almost any transition.

Figure 44 depicts the power traces for the TFET static XOR gate and the TFET differential style XOR gate. Obviously, the TFET CML XOR gate dissipates almost a constant power in contrast to the significant power overshoot of the static XOR gate. That is, the power profile of the TFET static XOR gate leaks more information for the attacker to identify the internal activity of the cryptographic system. However, the almost constant power consumption of a TFET CML XOR gate provides essentially no information about data transitions. Moreover, as discussed in the previous section that the $0 \rightarrow 1$ transition is essentially mirrored to a $1 \rightarrow 0$ transition in the CML gates, even though attackers may retrieve some information through the power glitches, it is very challenging for them to identify what the processing logic value is.
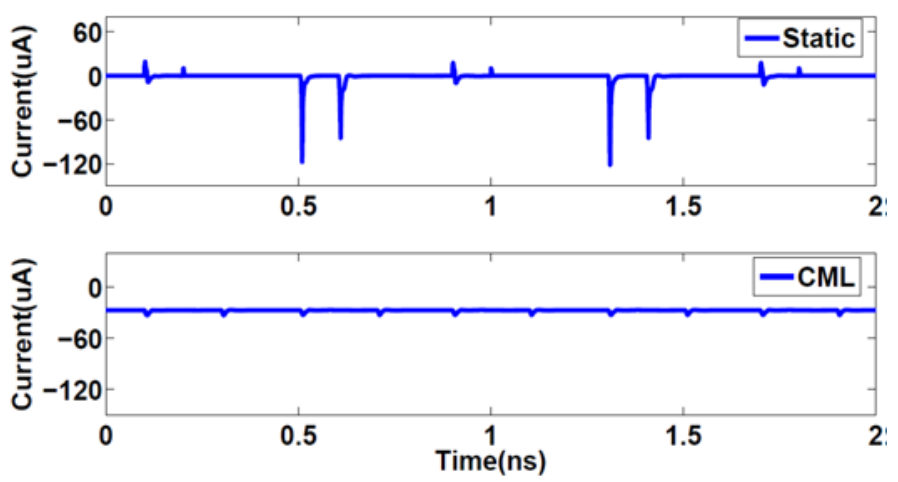

Figure 44. The power traces between TFET static XOR and CML XOR. 
Due to the large area and high power consumption, using CML to implement cryptographic hardware is not common-especially in lightweight cryptographic systems. To protect cryptographic circuits against DPA attacks, researchers often employ other techniques [115]. These solutions incur a significant computation cost where the cryptography already involves massive computation and consumes a relatively large power and area. As such, lower-power, TFET-based CML could be especially valuable when considering devices for the IoT, wireless sensor nodes, etc. Lacking an effective defense mechanism, hardware in these spaces can be substantially more vulnerable/susceptible to hardware attacks such as DPA. To address these challenges, we consider the impact of TFET-based CML on a 32-bit KATAN cipher. Here, a correlation power analysis (CPA) on KATAN32 is described to disclose the two key values. Initially, four selected plaintexts are loaded into the two registers and the 80 bit keys are all set to zero. Note that, in real cases, the key is the attackers' target and is unknown to attackers. When the start signal is received, KATAN32 begins encryption. Figure 45 shows the proposed CPA attack flow on KATAN32. Each selected plaintext and the hypothetical Subkeys $\mathrm{Ka}$ and $\mathrm{Kb}$ are calculated to achieve the intermediate values " $\mathrm{v}$ " matrix. Then, intermediate results are further calculated by the power model, which is defined as the Hamming weight model. The results from the Hamming weight model are defined as the hypothetical power consumption.

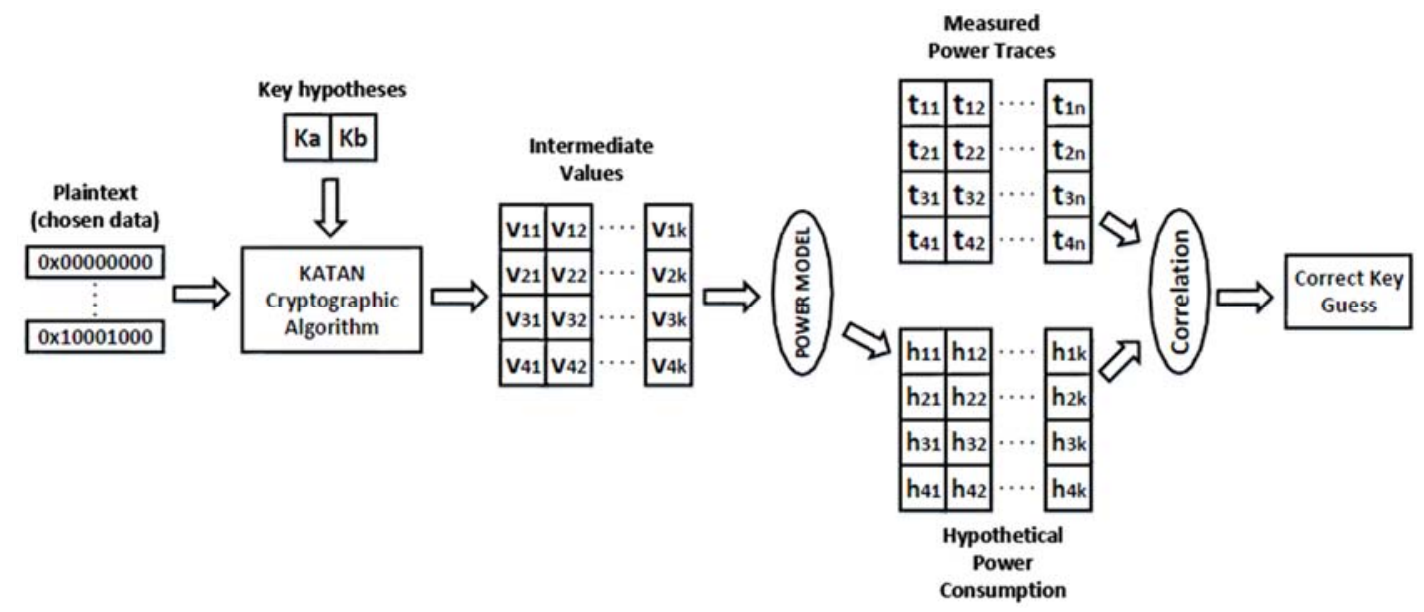

Figure 45. Correctional power analysis flow on the KATAN cipher.

The predicted power consumption is then compared with the measured real power consumption by the correlation coefficient formula as given in Equation (9). The highest correlation coefficient result stands for the correctly guessed keys. In this case, the keys ' $00^{\prime}$ reflect the largest correlation coefficient value. The next round follows the same mechanism, but with slightly different ciphertext, which is generated by the last round. Figure 46 shows the detailed correlation power analysis for the respective TFET static KATAN32 and TFET CML KATAN32 on one clock cycle. The black line describes the correct key value for subkeys $\mathrm{Ka}$ and $\mathrm{Kb}\left(=^{\prime} 00^{\prime}\right)$, which are the two most significant bits of the key. It is apparent that the correlation coefficient is largest for a static, TFET-based KATAN32 implementation when the correct keys are applied as shown in Figure 46a. By comparison, the correlation coefficient of TFET CML KATAN32 is more significant, and all four hypothetical keys are similarly distributed as shown in Figure 36b. Consequently, the TFET CML KATAN32 implementation is capable of successfully counteracting the correlation power analysis.

$$
\text { Corr. Coefficient }=\frac{\sum_{i=1}^{4}\left(t_{i}-\bar{t}\right) \cdot\left(h_{i}-\bar{h}\right)}{\sqrt{\sum_{i=1}^{4}\left(t_{i}-\bar{t}\right)^{2} \cdot \sum_{i=1}^{4}\left(h_{i}-\bar{h}\right)^{2}}}
$$




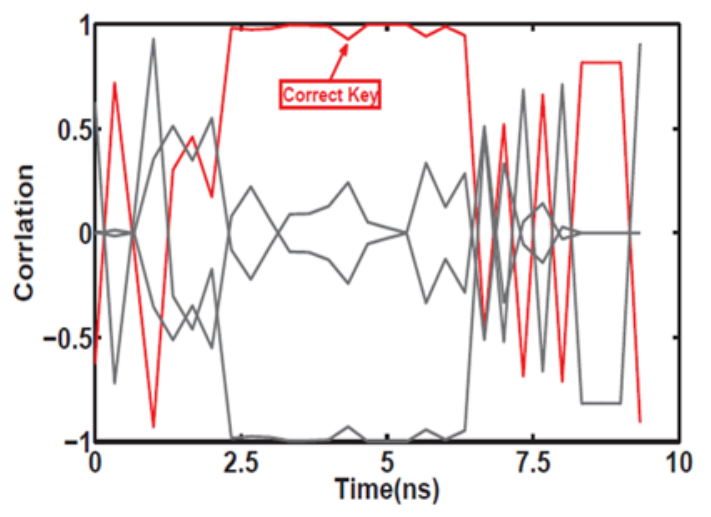

(a)

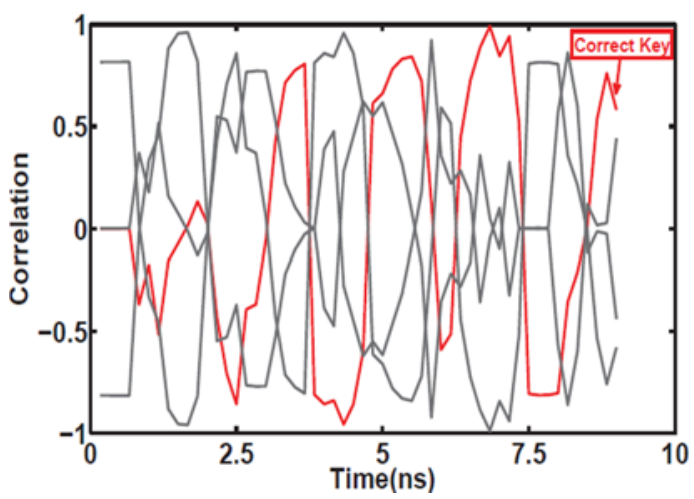

(b)

Figure 46. Correlation power analysis (CPA) attack on one clock cycle (a) TFET static KATAN32; (b) TFET CML KATAN32.

\subsection{Deception Techniques: Camouflage and Polymorphic Gates}

The two most severe attacks on IC manufacture are IP piracy and counterfeiting [116]. Several protection techniques have been proposed to prohibit an attacker from using reverse-engineering to know the scheme of the circuit, but the more popular one is camouflaging $[117,118]$. The camouflaging technique can protect the design at the layout level since each camouflaged gate can be programmed to different gates based on the designer configuration. Therefore, recovering the original circuit cannot be easily achieved using the reverse-engineering. However, implementing this technique using CMOS technology will significantly increase the area and the power penalties, especially for high level circuit security. In Rajendran et al. [119], a CMOS camouflaging standard cell utilizes 12 transistors and a group of contacts to achieve three logic functions, as shown in Figure 37. There are more contacts than in a normal standard cell, as some of the contacts work as dummies to camouflage the functionality of this logic cell. Three different logic functions can be produced by using these dummy and true different contacts. For example, if the fake contacts are 1, 3, 5, 7, 9, 10, 13, 14, 15, 18, and 19 and the true contacts are $2,4,6,8,11,12,16$, and 17, the camouflaging layout functions as a NAND gate. With more functionalities being achieved by a camouflaging gate, it becomes more difficult for attackers to recover the gate functionality through reverse-engineering. The area penalty of CMOS camouflaging layout ranges from 50 to $200 \%$ for 4 transistor NOR gates, 4 transistor NAND gates, and 8 transistor XOR gates.

Since the polarities in NMOS and PMOS are fixed, more transistors should be added to produce a camouflaging gate. Interestingly enough, the polarity signals in SiNW FETs can easily be modified and can therefore provide designers with an opportunity to switch the functionality of the gate without any extra hardware resources. For instance, Gaillardon et al. [37] employ four SiNW FETs to produce a NAND or an XOR gate. This one-tile layout includes four SiNW FETs, where circles stand for drain/source pins and bars represent the polarity gate (or control gate). Another proposed design has been presented to produce seven different types of gate by also using only four transistors but with different signal connections. Note that the functionality of the gate is fixed post-fabrication, with gate signals being connected to physical terminals. After these connections, the polarity gates perform as normal input gates, and no extra control circuitry is required to maintain the functionality. This design with the control polarity characteristic can be used to create camouflaging gates with much less performance overhead due to utilizing only four transistors. In fact, the additional polarity gate is leveraged in the camouflaging gate layout to reduce the transistor count. The overhead of this SiNW-based camouflaging layout is negligible, which is mainly caused by additional insignificant dummy contacts. Based on the aforementioned discussion, different logic gates could be produced using only two SiNW FETs, as shown in Figure 47a, where only 10 real and dummy contacts are 
adopted. More precisely, the scheme functions as a NAND gate if the $3,6,7,8$, and 9 contacts are connected as dummy. However, it will function as a NOR gate if the 1, 2, 4, 5, and 10 contacts are connected as dummy.

Another more complicated camouflaging gate with four different logic gates, $\mathrm{XNOR}, \mathrm{XOR}, \mathrm{NOR}$, or NAND, is demonstrated in Figure $47 \mathrm{~b}$. The four different functionalities can be achieved with the same input pins by changing the connections of the contacts and using only four transistors. In CMOS technology, 12 transistors are employed to achieve three different logic gates, XOR, NAND, or NOR gate. As a result, this scheme requires three times number of transistors compared to the SiNW structure shown in Figure 36b. However, five more contacts are used in the SiNW FET-based camouflaging gate, although the area overhead incurred by the extra contacts are negligible considering the transistor count reduction. To further evaluate the security improvement, the security metric has been used to check how easily an attacker can guess the full functionality of given designs containing camouflaging gates. In other words, if one camouflaging layout can achieve four functions, the chance that the attacker can retrieve the correct result is $25 \%$. Therefore, assuming that there are N SiNW FET camouflaging layouts incorporated in the design, the attacker may have to try up to $4 \mathrm{~N}$ times to obtain the correct design layout. As a consequence, it is promising that the SiNW FET-based camouflaging layout, which has more functionality and less area consumption compared to CMOS counterparts, can achieve a higher level of protection for circuit designs.

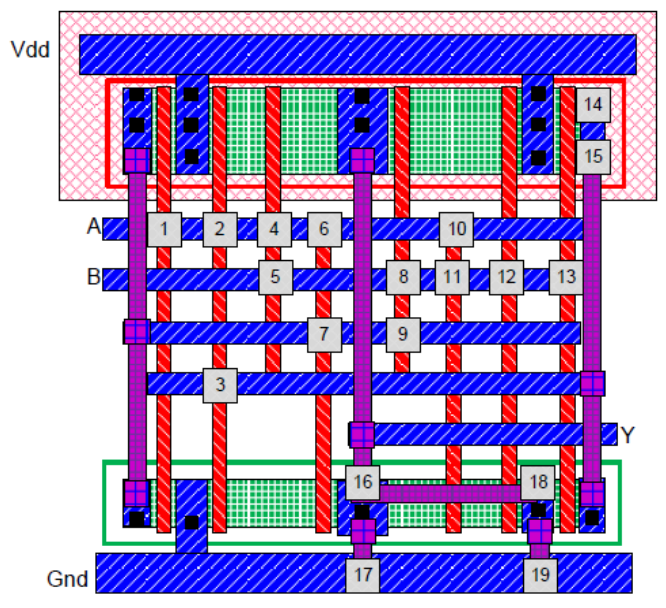

(a)

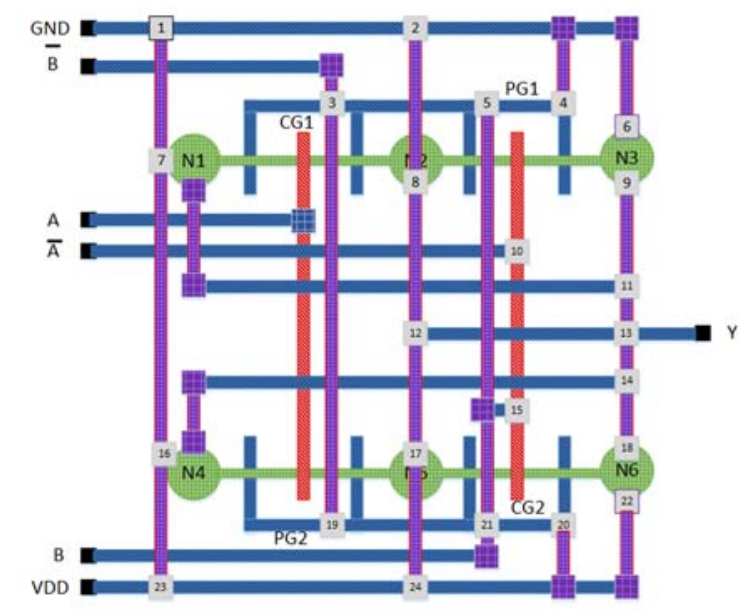

(b)

Figure 47. (a) Camouflage layout of CMOS logic gates Reproduced with permission from [119], Copyright ACM, 2013; (b) Camouflage layout of SiNW logic gates.

Polymorphic electronics, which were first introduced in Stoica et al. [120], are based on the idea of having multiple functionalities built in the same cell and deciding the input-output relation by means of a controllable factor in the circuit. For instance, a polymorphic gate presented in Stoica et al. would be an AND gate when the VDD is $3.3 \mathrm{~V}$ and function as an OR gate when VDD is lowered to $1.5 \mathrm{~V}$. Such multifunctional gates would prove useful in a number of applications. Circuits that change functionality with temperature variation can find use in aerospace applications, or those that respond to VDD variation could be used to change functionality when the battery is low. In addition, polymorphic electronics could prove useful in evolvable, intelligent, or self-checking hardware. For security purposes, adding polymorphic gates to a digital circuit can hide the real functionality of the circuit. Since the circuit functions correctly only in a certain configuration of the control signals known to the designer, even if the adversary knows the whole netlist (including the dummy and true contacts), he or she will not be able to utilize the circuit in his or her own design. Carefully encrypting a logic in this way can ensure that it will take too long for the adversary to find the key (a vector constructed from all morphing signals of the polymorphic gates). Therefore, the polymorphic gate becomes a good 
candidate for integrated circuits protection against IP piracy. Traditionally, several CMOS-based polymorphic gates have been reported with different control methods, such as temperature, VDD variation, and external signal level. Stoica et al. [120] designed polymorphic gates by an evolution algorithm. However, the circuits face issues during simulation, as the circuit was evolved to satisfy certain constraints that do not include all aspects of a complete design. For example, the NAND/NOR polymorphic gate based on an external signal will experience states where the transistors have to compete over the output, causing the circuit to draw a constant current through those paths. Further, since inputs may be shorted to ground or VDD during certain states, it is difficult to connect multiple stages of these gates in sequence. The circuit based on VDD variation is the most practical solution and was fabricated; however, redesigning it in newer technologies where the VDD range is limited would be a difficult task. Another promising solution presented in Ruzicka [121] is a NAND/XOR gate. The proposal requires nine transistors, where the functionality can be changed using an external signal. The performance of the gate is good even when we redesigned it in the $22 \mathrm{~nm}$ FinFET technology node.

Here, a novel approach to designing polymorphic gates using polarity-controllable FETs is proposed [122]. The ability to control the polarity of a transistor enables us to build polymorphic cells with a much lower number of transistors. The basic NAND and NOR gate structure is similar for both the CMOS and the SiNW FET. The polarity control gate does not reduce the number of transistors required to implement NAND and NOR using SiNW FET technology. However, this unique property allows us to change the functionality of the gate simply by interchanging the VDD and GND. Note that interchanging the VDD and GND connections in any CMOS-based logic will produce the complement of the original function at the output, but full voltage swing at the output will not be achieved due to the presence of NMOS and PMOS in the pull-up network and pull-down network, respectively. Therefore, using this method, one can gather the VDD and GND terminals of the NAND and NOR gates in a combinational logic into a vector and construct a "logic encryption key". As opposed to the work presented in Rajendran et al., which adds additional XOR or XNOR gates into a logic gate to realize the logic encryption scheme and thus incurs performance overhead, this approach has zero overhead in terms of gate count and trivial wiring cost due to the switching of VDD/GND. Figure 48 presents an example of the conversion of a digital circuit to its polymorphic gate equivalence.

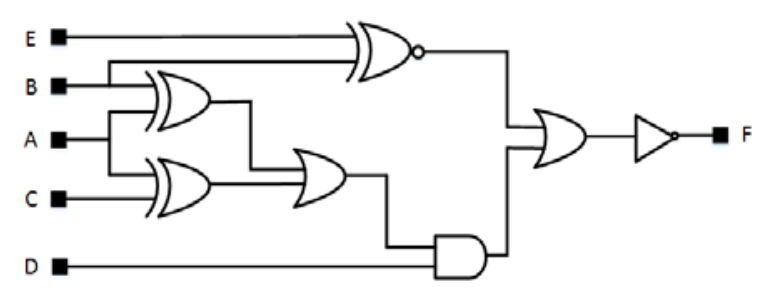

(a)

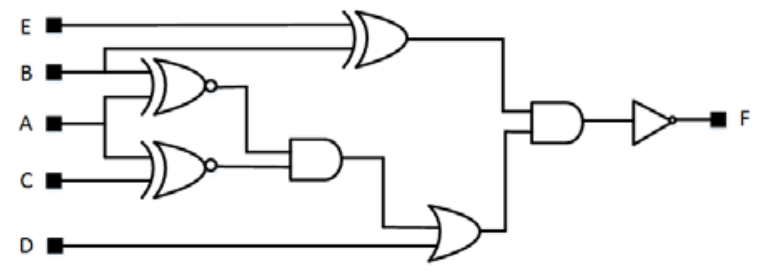

(b)

Figure 48. A digital logic gate schematic (a) original design; (b) after polymorphic gate conversion.

\subsection{Logic Locking Using Silicon Nanowire FETs}

Applying logic encryption technique on real chips might be infeasible, especially for high security level purposes since the performance overhead will be high. This overhead could be reduced significantly if a designer replaces some of the gates in the original circuit with polymorphic gates designed using SiNW FETs, instead of adding additional key-gates, e.g., XOR/XNOR or AND/OR gates or multiplexer. Moreover, in all of the previous works, there is only one key-bit for each key-gate insertion. To successfully prevent attackers from using the brute force search, the secret key length of the encrypted design should be large enough, e.g., larger than 128 bits. Increasing the size of the secret key leads to increase the overhead largely, which might be larger than the size of the original netlist. Interestingly, using SiNW polymorphic gates, the designer can enlarge the key size up to 6x for any simple 2-input gate if the keys are not gathered in a line for each exchanged gate. 
Adding an inverter to create a uniform key-bit will not increase the circuit overhead very much. Figure 49 shows the use of SiNW polymorphic gates for an encrypted combinational benchmark circuit. When both $\mathrm{K} 1$ and $\mathrm{K} 2$ are set to zero, the correct functionality of the design is revealed. However, if one or both of the secret key of the polymorphic gate(s) is set to ' 1 ', incorrect functionality is produced. More specifically, the correct output " 00 " is revealed for the circuit shown in Figure 49 if the input pattern " 01000 " is applied. In contrast, if the value of the two keys set to ' 1 ' with the same input pattern, the output of Figure 49 will be "11" since the two polymorphic logic gates are switched NOR gates. Furthermore, an incorrect output of " 11 " or " 01 " will result if one of the polymorphic gates is reprogrammed to a NOR gate via making K1 or K2 equal ' 1 ', respectively. As a consequence, three wrong keys will produce two corrupt outputs, whose Hamming distance of $50 \%$ and $100 \%$, compared to correct output patterns, is achieved. Besides the NAND/NOR polymorphic gate, two other possible polymorphic gates can be presented, which are AND/OR and XNOR/XOR polymorphic gates. Incorporating different number of the polymorphic gates will increase the protection level of the design [123].
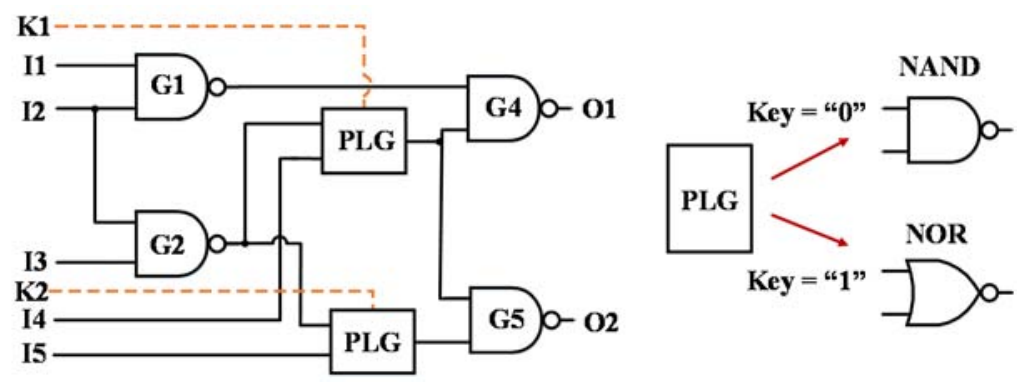

Figure 49. Encrypted ISCAS circuit with NAND/NOR polymorphic gates.

\subsection{Emerging Memory Security}

The spin-based devices have been used in different security applications, such as strong PUF $[124,125]$ and true random number generator (TRNG) [126], which are hardware primitives. However, this does not mean that these devices and their applications are fully reliable. In fact, these devices can be attacked by manipulating their associated parameters, such as magnetic field and temperature. Additionally, their non-volatility feature can be leveraged by an attacker to damage data or retrieve sensitive information (such as password or cryptographic keys) when the device is off. Therefore, they have new security vulnerabilities that were not present in conventional SRAM and embedded DRAM [127]. As an example, the state of the MTJ magnetic layers or the domain walls (in the DWMs) can be altered by manipulating the spin-polarized current (based on the degree of spin) or an external magnetic field (based on its magnitude/polarity). The force of manipulation should be sufficient enough to flip a weak bit in the presence of process variations and ambient disturbances. In this regard, securing these systems and protecting their data integrity in front these malicious attacks is critical. The attacks may consider different scenarios for compromising data privacy.

In an example scenario, when the tag bits are constant throughout the power cycle, a malicious read operation can cause a cache hit in an NVM last-level cache (LLC) with the purpose of leaking sensitive information such as keys, passwords, and account numbers. In this scenario, a larger cache is more vulnerable since it presents more data for leakage. Many solutions have been proposed for the protection of memory systems such as data encryption. Besides the discussed threats, the reliability issues of the MTJ device [128] may also be leveraged by an adversary to perform malicious actions. A reliability issue can be maliciously created by inducing malicious aging and/or malicious process variations. For further considerations, it is assumed that all the dynamic reliability management/aware mechanisms are disabled (by inserting a hardware Trojan). In order to model this attack, the free layer thickness (Tm) of perpendicular magnetic anisotropy (PMA)-based MTJ is maliciously varied 
using the SPICE models for magnetic tunnel junctions based on mono-domain approximation [129]. This malicious variation is realized by the insertion of a ferromagnet with an incorrect thickness for the free layer. In an alternative strategy, a ferromagnet with the same size but different material may be used to enforce a similar effect. The possible practical demonstrations for this action can be stated as follows: (1) inside the untrusted foundry by physical intrusion, (2) doing modifications within the algorithms used for sizing the design cells, and (3) inserting a few maliciously constructed cells in the process of IC design flow [130,131]. The impact of this attack can be observed as the occurrence of logical transitions of the MTJ device earlier or later than the expected time. This can cause probable performance degradation (mild case) or logical state sensing and propagation throughout the system (severe case). A common technique for detecting (and correcting) functionality failures is run-time monitoring (and reacting). Accordingly, a built-in-self-test module for reliability-related security (BIST-RS) analysis. The functionality of this module can be classified into (a) error detection, (b) error prediction, and (c) error masking. The "error detection" process is described as monitoring the signals of logical paths for transitions after the clock edge and flagging a possible error. Figure 50 displays a BIST-RS architecture for the reliability-related security analysis of the MTJ device. The architecture is expected to detect maliciously sized MTJ cells. The three main elements in this architecture are as follows: a data encoder, an MTJ structure (i.e., an array of the MTJ cells), and a data decoder [132]. The data encoder is responsible for making the sender message that is constructed by the applied test pattern and its calculated fingerprint. The MTJ structure is responsible for correctly transmitting information to the receiver and preserving its integrity. In other words, the logical state of each MTJ cell in the structure should remain the same or a transition needs to occur depending on its corresponding bit in the applied test pattern. A single malicious MTJ cell with its value of free layer thickness that is outside of the acceptable range causes an alteration in the information. The receiver message that comes from the MTJ structure is checked and the integrity verified by the data decoder. The error signal indicates whether the MTJ cells are healthy or not.

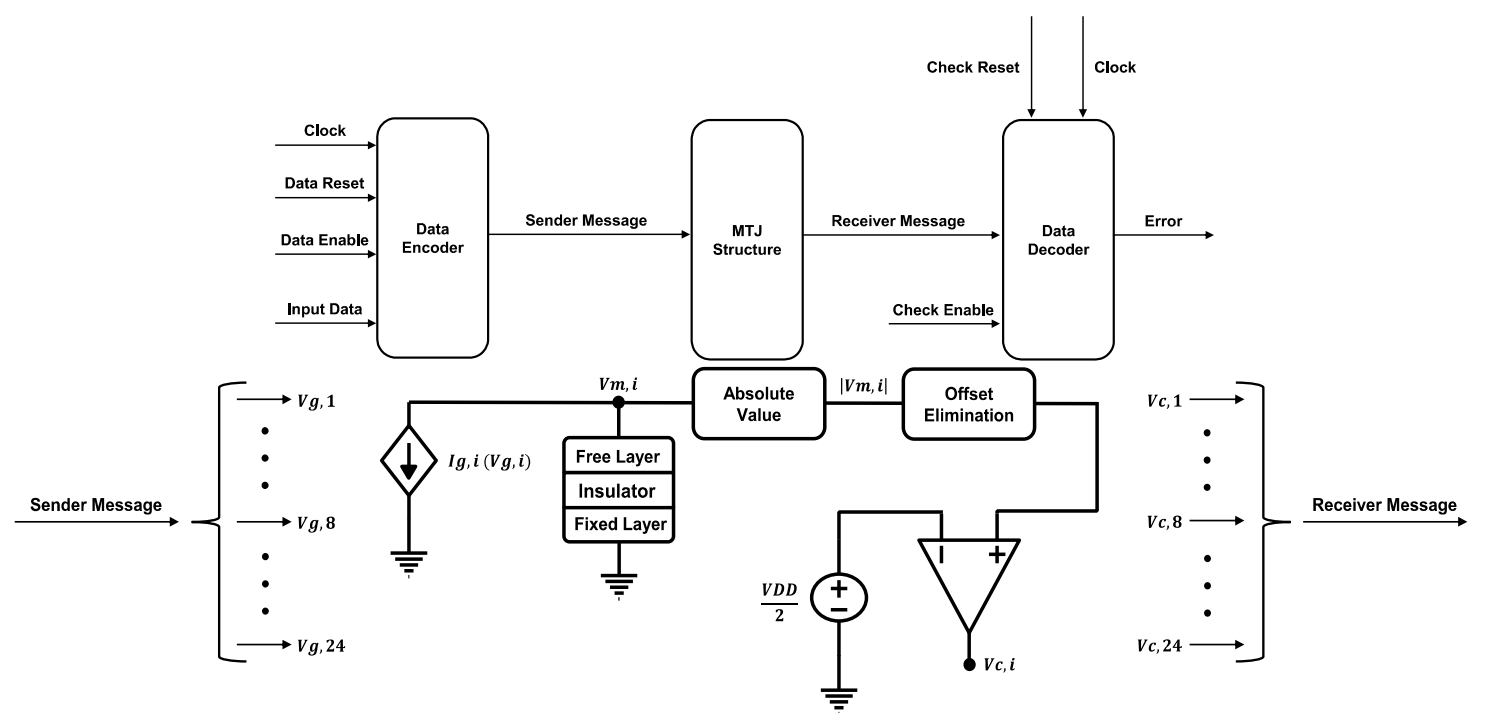

Figure 50. BISR-RS architecture for the MTJs under attack.

Due to the limitation of the PCM cells in the number of write operations (which is usually a maximum of $10^{7}-10^{8}$ ), they can be vulnerable to a write attack. According to the attack, a malicious person can repetitively write to some addresses in the memory for wearing out the cells (requiring $30 \mathrm{~s}$ for each [133]) and consequently causing failure in the memory system. Additionally, the non-uniformity of the memory write pattern can worsen this situation even further. A few countermeasures have been proposed for the non-volatile memories. The authors of [134] proposed a nonvolatile main memory ( $i$-NVMM) module that performs selective data encryption using the 
AES algorithm. This module only encrypts time-based unused data (which are the data that are not frequently accessed during run-time execution) for the aim of reducing timing and power overheads. The problem with this technique is exposure of the data when intrusion occurs during run-time operation. According to [135-137], the counter-mode XOR-based encryption in the AES algorithm can be modified to calculate a crypto-PAD for each memory line. In this way, run-time data protection is provided for all data in the NVMs with insignificant timing and power overhead. The authors of [138] offered a countermeasure for the PCM write threat according to which either the number of write operations is reduced or a "wear-leveling" is used to "write uniformly". A few examples of wear-leveling methods include the randomized region-based Start-Gap [139], the multi-level Security Refresh [140], and Online Attack Detection [141]. These methods suffer from high write or extra hardware overheads due to their frequent need in swapping data for speeding up the process of remapping logical to physical addresses. Additionally, this process increases access delay, wears out the storage cells, and may suffer from uneven memory sub-spaces (due to having partial leveling and limited mapping). A solution called, multi-way wear-leveling (MWWL) was proposed by Yu and $\mathrm{Du}$ [142] according to which a uniformly distributed writes to the entire physical address space is specified. In other words, the logical address space is divided into equally sized sub-spaces (or "ways") and each sub-space is responsible for its own remapping process and wear-leveling of its own addresses. Due to the small size of logical space, the physical space under write changes more frequently and remapping of an address under attack can occur with a smaller speed. The physical space under write can be as large as the entire memory address space.

As another countermeasure, Young et al. in [143] introduced Dual Counter Encryption (DEUCE) technique according to which the write-back changes are monitored and only the changed words are encrypted for the goal of improving the memory performance and lifetime. The wear-leveling methods usually remap logical addresses to physical addresses randomly and dynamically. However, this does not mean that they can be fully trusted. Mao et al. realized that the details of address remapping can be revealed through monitoring NVM row buffer hits [144]. A row buffer hit can unfold a logical address mapped to a certain physical row. The new logical addresses mapped to the same row can be similarly revealed. A countermeasure for this attack is Intra-Row Swap (IRS) according to which the mappings are changed and the actual physical addresses are concealed. In other words, the position of memory cells is obfuscated.

\subsection{Low-Power SAR ADC Security Using Emerging TFET Technology}

The security aspects of analog and mixed-signal circuits have less been studied [145-148]. The ADC as a well-known and widely applicable mixed-signal module in the IoT world can be a target for malicious operations by adversaries. The malicious operations on an ADC can be Hardware Trojan (HT) insertion, piracy of digital and analog/mixed-signal intellectual properties, overbuilding of integrated circuits, reverse-engineering, side-channels analysis, and counterfeiting. Therefore, this module, in its design, fabrication, installation, and operation life processes, must be secured and protected. In here, the security of SAR ADC with the threat of Hardware Trojan is discussed.

According to [149], there are two critical points in a central processing unit that are the subject of sabotage by HT insertion: the data path and the control unit. An ADC can be attacked by targeting the same points on its circuit and inserting an HT inside the register file (which is a digital IP) and inserting an HT inside the sample/hold/compare (which is an analog IP). The Trojans have the aim of damaging the ADC functionality "sometimes". In order to justify the steeliness of the proposing Trojans, it is assumed that each of them is activated by a "Main Trigger" and a "Mate Trigger". This means that, when the two trigger signals are active, the Trojan becomes on. The "Main Trigger" of each Trojan is constructed based on making its behavior sneaky and random.

The "Mate Trigger" for each Trojan is generated by other parts of the System on a Chip (SOC) design becomes active only during the "chip run-time operation" based on the running application. This scenario reduces controllability and observability on the Trojan circuit; consequently, it is less 
likely to be detected. For each of the Trojans, a countermeasure is proposed as well. It is expected that the number of logical cells used in the implementation of each of these Trojans, compared with the total number of logical cells within the chip, may be desirable. In another implementation scenario, the unused logical cells during the run-time operation can be identified and used for the construction of the Trojan circuit using a predefined adaptive mechanism. The same concept may be applied for the implementation of the defense circuit.

The inserted Trojan for the register file manipulates the exiting signals of the D-type flip-flops sometimes and is called the data-path threat model. Figure 51 shows the Trojan circuit according to which two of the flip-flops are randomly selected. The output signals of these flip-flops are shuffled by their corresponding unit depending on the logical state of the Select signal, which is generated by a frequency divider. The frequency divider is controlled by two signals: (a) the sampling clock signal (CLKS/H) and (b) the last value of the Trojan enable signal (Trojan_En). The Trojan_En signal activates the Trojan that causes inversion of the stored data in a chosen flip-flop using a multiplexer. The chosen flip-flop in this work is the third bit that creates a medium-level error.

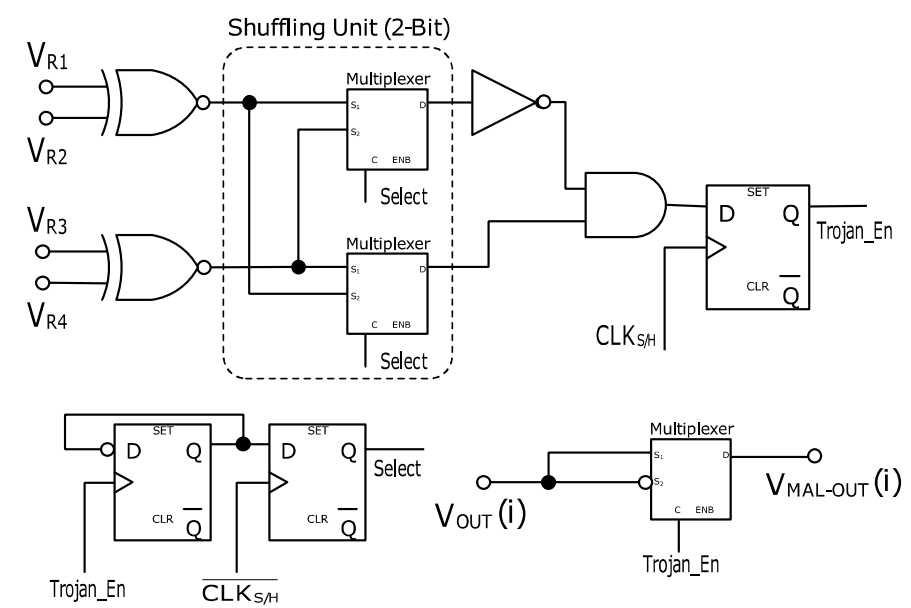

Figure 51. The circuit for the date-path-based attack.

A convention is assumed for the quantized signal by the ADC according to which the standard waveforms (for example, ramp, sine, sawtooth, and triangular) usually have \pm 1 least significant bit (LSB) difference between their adjacent sampled data points. This means that the digital code for a certain data point is +1 LSB higher, the same, or -1 LSB lower than the last data point. This convention is taken into account in monitoring and security checking the ADC. If the quantized signal and the ADC operation does not follow this convention, then the defense circuit flags an abnormal condition. Flagging an abnormal condition is followed by notifying the user and sending out the last correct code. The circuit for practical realization of this mechanism is shown in Figure 52. In this circuit, IN(5:0) represents the ADC output bits before processing and OUT(5:0) represents the ADC output bits after processing. The Cond 1 signal becomes equal to logic one when an unusual condition occurs. The registers hold the possible cases for evaluation of the next sampling and provide synchronization in the defense operation. Other advantages of the defense circuit include the following: (a) they help to attenuate the output noise, and (b) the output signal is filtered and smoothed. The added circuitry causes a delay in receiving the output bits. 


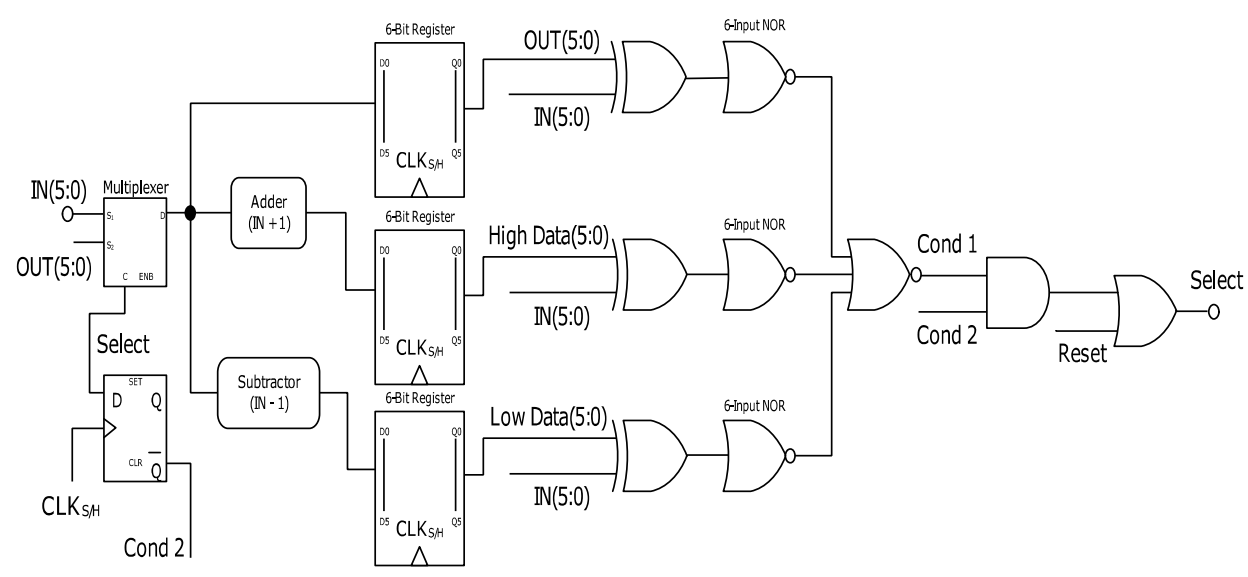

Figure 52. The circuit for the date-path-based countermeasure.

In order to attack the control unit, the capacitor-connected switches within the sample-hold-compare (SHC) block are manipulated. All the capacitors in this block should be connected to common-mode voltage when the sampling process is started. Depending on the coming control signals, they are connected to either the supply voltage or the ground. The attack aims to disable the connection of one or more of the capacitors to the common mode voltage at the time of sampling sometimes. In this way, the victim capacitor holds its charge from the last sampling and consequently one or more number of output bits may be different than what they supposed to be. Figure 53 shows the Trojan circuit for this attack. The flow of this circuit can be described in this way: (1) The output of the comparator within the SHC block triggers a four-bit counter. (2) The counter output signals can construct up to 16 Boolean functions using a four-bit Minterm construction unit. The chosen functions are the 4 th, 7 th, 12th, and 14th rows of the corresponding truth table. (3) The outputs from the Minterm construction unit are sent to a shuffling unit. The shuffling unit is made of multiplexers and the select signals for them are Choice(2:1) that are taken out from any part of the circuit such as the SHC block. In order to make the choice signal, the exclusive-OR (XOR) function is run on the "even" and "odd" bits of the ADC output. (4) The exiting bits from the shuffling unit are stored in a four-bit register. This register is triggered by the sampling clock. (5) The control signal for one of the capacitor-connected switches becomes inactive (which means equal to zero) depending on the stored value in its respective flip-flop in the four-bit register. This may lead to the generation of an incorrect value by the analog comparator within the SHC block. In this work, the 2nd-5th bits of the ADC output are selected for malicious alteration.
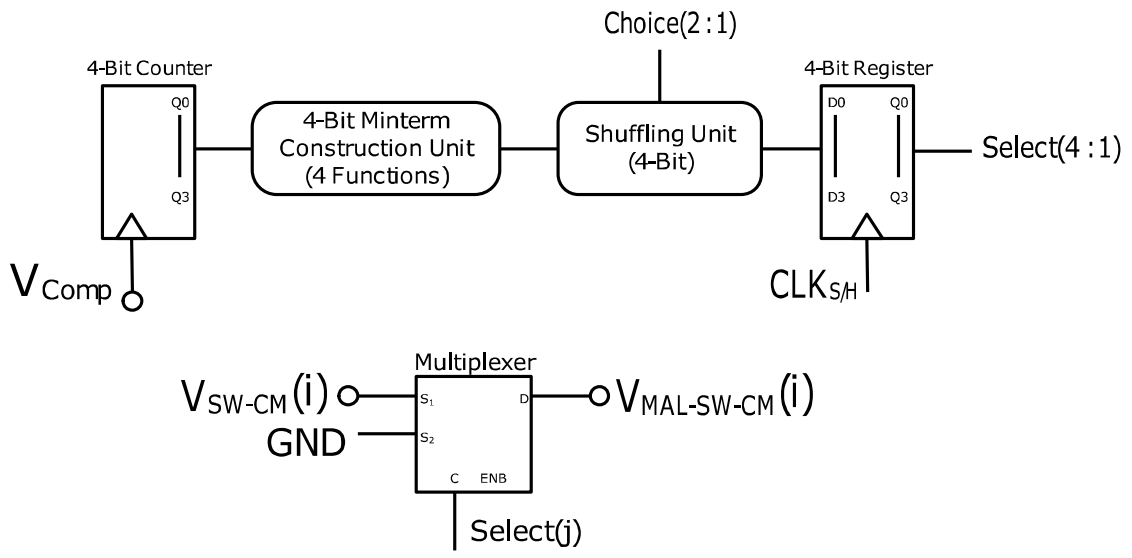

Figure 53. The circuit for the control-path-based attack. 
A common technique in designing a Built-in-Self-Test (BIST) module for an IC is "sub-circuit replication" [150]. A BIST module can be externally inserted or internally developed (from the available design cells in a certain chip mode). Here, the countermeasure for the control-based threat is a trustworthy and possibly lightweight replication of the SHC analog block along with a decision unit. The decision unit has the responsibility of comparing the coming signals from the possible victim SHC and the trustworthy SHC. If this unit determines an error, then the user is notified and the output signal of the trustworthy SHC is given to the register file. This action may bring performance degradation and quality decay due to the differences between the actual SHC and the trustworthy SHC block, but it certainly delivers correct functionality. The circuit for the countermeasure can be seen in Figure 54. In this circuit, $\mathrm{V}_{\mathrm{REF}}$ is the trustworthy SHC output signal, $\mathrm{V}_{\mathrm{MAL}}$ is the possible victim (or deterministically malicious) SHC output signal, and $\mathrm{V}_{\mathrm{O}}$ is the delivering output signal by the decision unit. Whenever a mismatch occurs between the two mentioned signals in the "timing status" and the "logical status", the error signal becomes equal to logic one and the $V_{\text {REF }}$ is delivered to the register file.
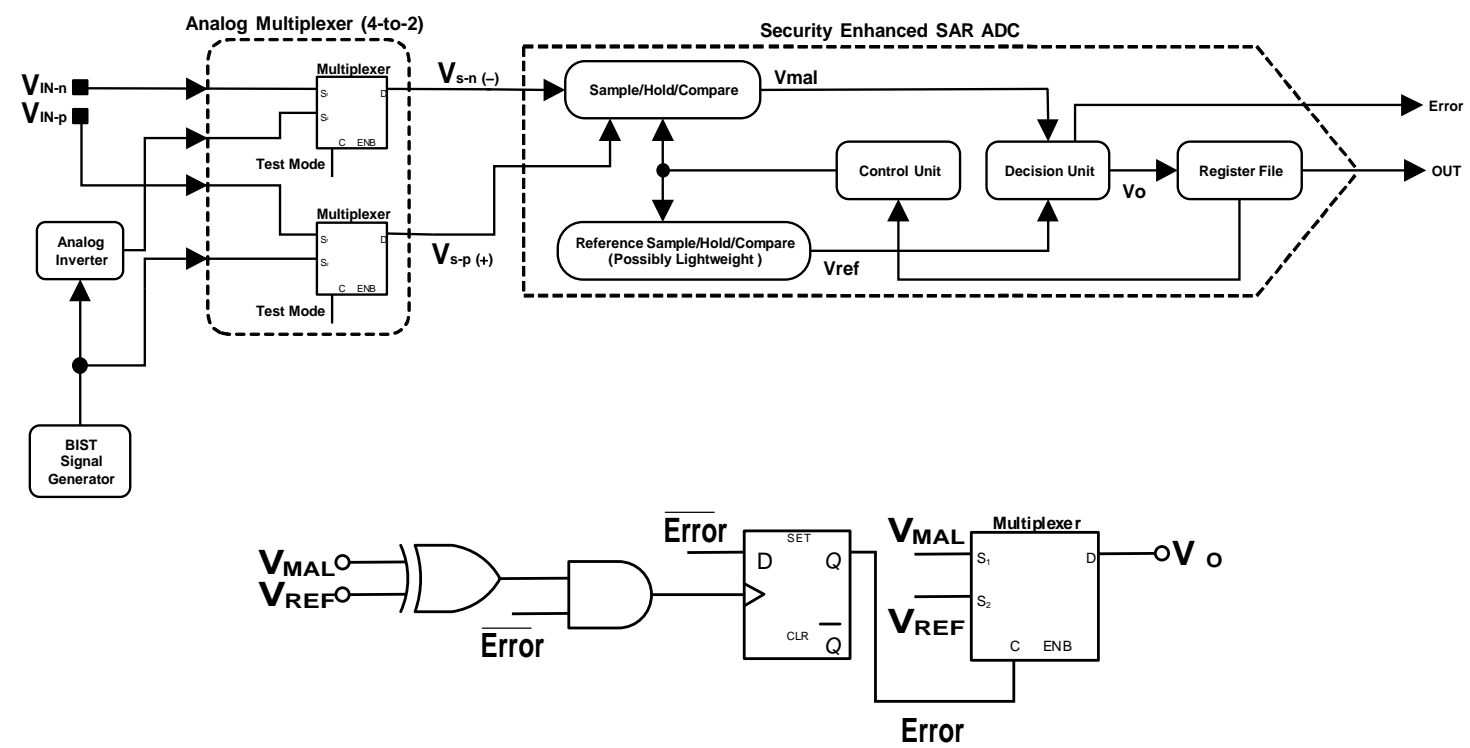

Figure 54. The circuit for the control-path-based countermeasure.

In order to assess the effects of the discussed attacks on the ADC operation as well as evaluating the effectiveness of their countermeasures [151], five different operating conditions are defined for analysis: (a) when the ADC is in healthy condition; (b) when the ADC is under the data-path-based attack; (c) when the ADC is under the data-path-based attack, but it is defended by its corresponding countermeasure; (d) when the ADC is under the control-based attack; and (e) when the ADC is under the control-based attack, but it is defended by its corresponding countermeasure. The used device for implementing all the discussed circuits is a tunnel field effect transistor with a $20 \mathrm{~nm}$ channel length, and the employed simulator is the Cadence Spectre Circuit Simulator. The type of analysis is transient and its duration is $120 \mathrm{~ms}$, the frequency of system clock is set to $20 \mathrm{MHz}$, all the capacitances in the SHC block are specified according to their indices in the capacitor array as well as the value of the base capacitance that is equal to $20 \mathrm{fF}$, and the supply voltage is equal to $0.3 \mathrm{~V}$ in all of the performed simulations. Due to the fact that a full scale ramp input signal is an ideal waveform in testing ADCs because of its feature in producing all the possible codes, it is used here for functionality evaluation. The applied ramp signal has the maximum amplitude of $0.3 \mathrm{~V}$. The starting point of its slope is at $5 \mathrm{~ms}$ and the ending point is at $87 \mathrm{~ms}$. Figure 55 shows the simulation results according to which the ADC functionality in the five operating conditions can be analyzed. According to the results, the control-based Trojan has more detrimental impacts since it brings both large and small variations in 
the reconstructed analog signal from the ADC output, while the data-path-based Trojan causes only a few large variations. The capability of the countermeasures in eliminating the impacts of attacks is acceptable.

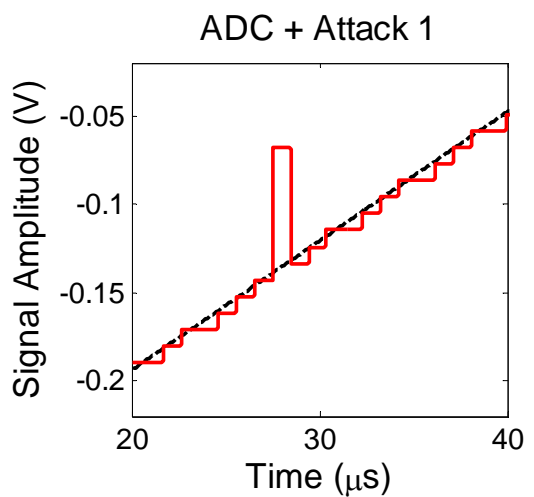

(a)

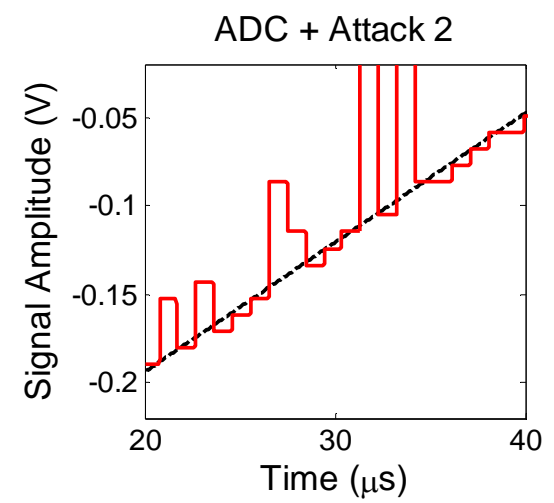

(c)

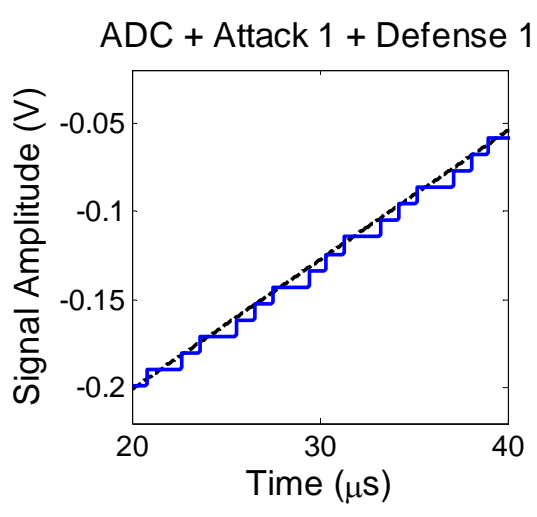

(b)

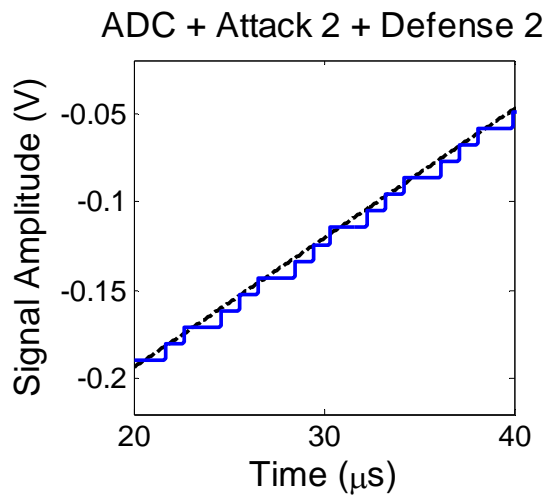

(d)

Figure 55. The functionality analysis of the SAR ADC in the last four operating conditions: (a) Attack 1; (b) Attack $1+$ Defense 1; (c) Attack 2; (d) Attack 2 + Defense 2.

\subsection{Spiking Neural Network Security}

Running a spiking neural network on an embedded device, though embracing superior energy efficiency, introduces security issues. For example, the attacker can pirate the learning algorithm by observing the outputs of the system using various input patterns. The possible attack model is explained as follows: An attacker can reverse-engineer to understand the hardware implementation of the system. Since the attacker does not know the algorithm implemented by the hardware, he/she can choose an arbitrary model. Besides the original model, he/she could also use another learning algorithm as the replicated model to learn the function. Moreover, it is not necessary to select the same model as the original one to obtain reasonable prediction and accuracy. The comparison between original learning support vector machine (SVM) model and other replica models is shown in Figure 56 [152]. 


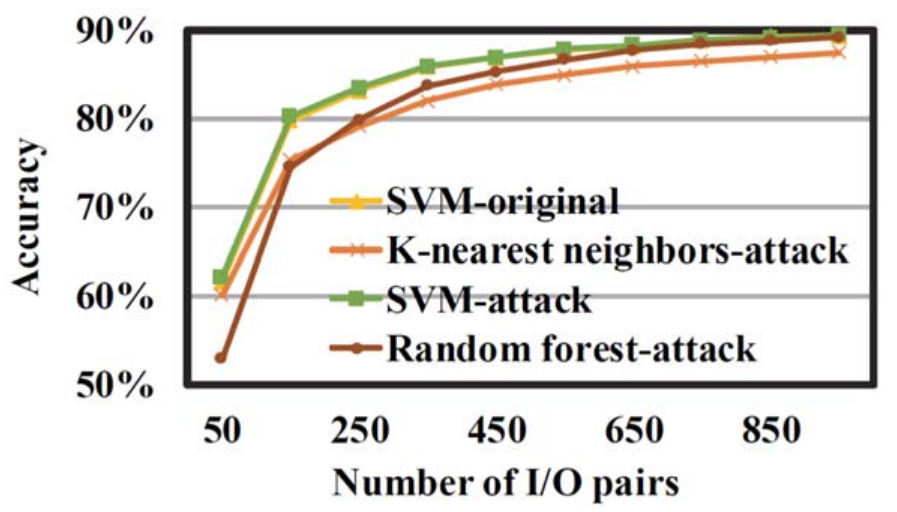

Figure 56. Comparison of learning accuracy among the original model and other learning models.

To prevent the attacker from learning the function of the model behind the system, the obsolescence effect of memristors is utilized [152]. The resistance of a memristor gradually changes on applying voltage pulses, eventually leading to the ON state or the OFF state. The obsolescence effect is called as the original resistance value "vanishes" on applying a voltage pulse. Figure 57a,b show both naïve and revised design using memristor arrays. The memristors in Matrices M1 and M2 are changing in the opposite direction.

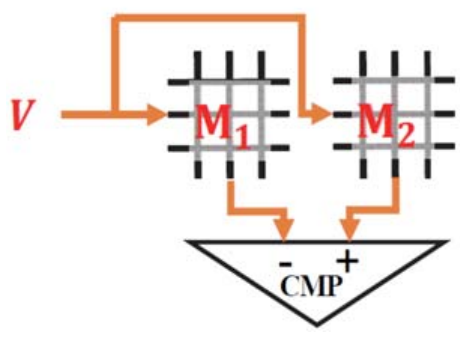

(a)

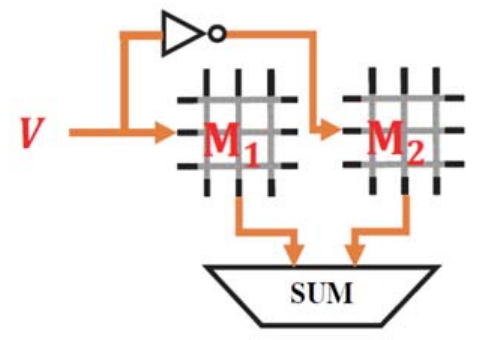

(b)

Figure 57. (a) Naïve design with a positive voltage applied to both crossbar arrays and (b) revised design with a positive voltage applied to the first crossbar array and a negative voltages applied to the second crossbar array.

With the obsolescence effect of memristors, the naïve design shows a linear degradation and the revised design shows a nonlinear degradation. Figure 58 displays the accuracy of different databases using the replica model for different defensive designs. The revised design is more resilient against replication attack.

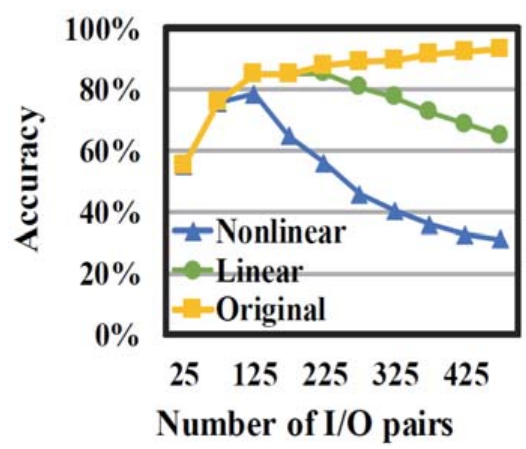

(a)

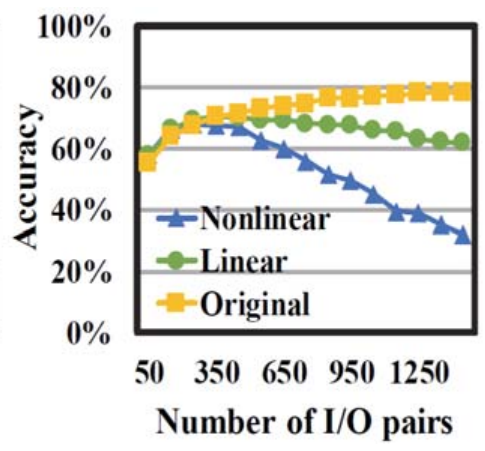

(b)

Figure 58. Cont. 


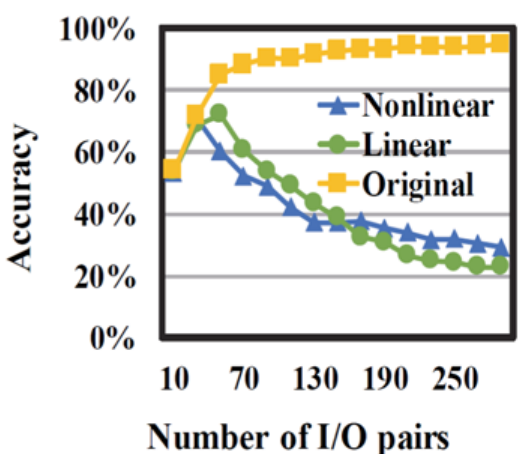

(c)

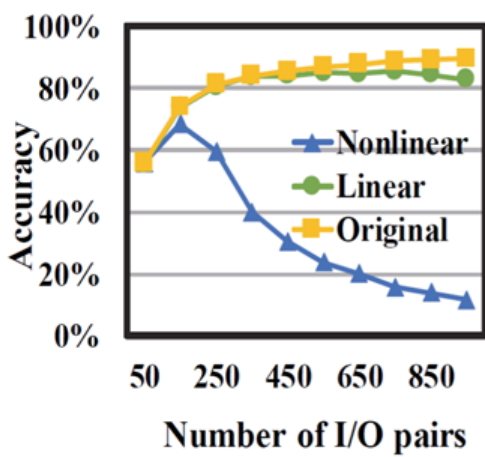

(d)

Figure 58. Accuracy between naïve and revised designs for (a) Digit, (b) Faults, (c) Image, and (d) MNIST benchmarks.

\section{Summary}

In this review, a broad range of low-power designs using emerging logic and memory technologies has been discussed. Emerging non-volatile memories and steep sub-threshold slope devices beyond CMOS are presented. Low-power SAR ADC design using tunnel FETs for IoT sensors is presented. Hybrid $\triangle \Sigma$ SAR ADC to increase signal-noise dynamic range and the equivalent number of bits resolution for low-power IoT is also introduced. Bio-inspired neuromorphic computing using stochastic neurons and memresitor synapses for ultra-low-power computing in an unsupervised manner is also illustrated. Hardware security including light-weight KATAN encryption for correlational power analysis, logic locking using SiNW and ASL devices against SAT attacks, deception techniques such as camouflage layout, obfuscated polymorphic gates, split manufacturing, and SAR ADC Trojan detection and countermeasures have been highlighted. Finally, bio-inspired neuromorphic computing security is briefly discussed.

Acknowledgments: The authors wish to thank Yu Bi for his early contribution on silicon nanowire camouflage, KATAN light-weight encryption and correlation power analysis. This work is supported in part by the Florida Center for Cybersecurity $\left(\mathrm{FC}^{2}\right)$.

Author Contributions: Jiann-Shiun Yuan organizes the materials and writes the manuscript. Jin Lin contributes to low power SAR ADC and hybrid $\triangle \Sigma$ SAR ADC designs. Qutaiba Alasa makes a contribution in polymorphic gate logic locking using silicon nanowire and all spin logic devices. Shayan Taheri contributes to SAR ADC Trojan attacks and countermeasures. All authors proofread the manuscript.

Conflicts of Interest: The authors declare no conflict of interest.

\section{References}

1. Bauer, H.; Patel, M.; Viera, J. The Internet of Things: Sizing up the Opportunity, Mckinsey \& Company. Available online: http:/ /www.mckinsey.com/industries/semiconductors/our-insights/the-internet-ofthings-sizing-up-the-opportunity (accessed on 15 May 2017).

2. Auth, C.; Cappellani, A.; Chun, J.; Dalis, A.; Davis, A.; Ghani, T.; Glass, G.; Glassman, T.; Harper, M.; Hattendorf, M.; et al. 45nm high-k + metal gate strain-enhanced transistors. In Proceedings of the Symposium on VLSI Technology, San Jose, CA, USA, 21-24 September 2008; pp. 128-129.

3. Chang, V.; Ragnarsson, L.; Pourtois, G.; O'Connor, R.; Adelmann, C.; VanElshocht, S.; Delabie, A.; Swerts, J.;

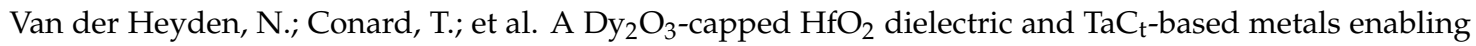
low- $\mathrm{V}_{\mathrm{t}}$ single-metal-single-dielectric gate stack. In Proceedings of the International Electron Devices Meeting, Washington, DC, USA, 10-12 December 2007; pp. 535-538.

4. Chang, L. Exteremly scaled nano-CMOS devices. Proc. IEEE 2003, 91, 1860-1873. [CrossRef] 
5. Wu, C.; Lin, D.; Keshavarzi, A.; Huang, C.; Chan, C.; Tseng, C.; Chen, C.; Hsieh, C.; Wong, K.; Cheng, M.; et al. High performance 22/20nm FinFET CMOS devices with advanced high-K/metal gate scheme. In Proceedings of the 2010 International Electron Devices Meeting, San Francisco, CA, USA, 6-8 December 2010.

6. Seok, M.; Chen, G.; Hanson, S.; Wieckowski, M.; Blaauw, D.; Sylverster, D. CAS-FEST 2010: Mitigating variability in near-threshold computing. IEEE Trans. Emerg. Sel. Top. Circuits Syst. 2011, 1, 42-49. [CrossRef]

7. Farooq, M.G.; Graves-Abe, T.L.; Landers, W.F.; Kothandaraman, C.; Himmel, B.A.; Andry, P.S.; Tsang, C.K.; Sprogis, E.; Volant, R.P.; Petrarca, K.S. 3D copper TSV integration, testing and reliability. In Proceedings of the International Electron Devices Meeting, Washington, DC, USA, 5-7 December 2011.

8. Devadas, V.; Aydin, H. On the interplay of voltage/frequency scaling and device power management for frame-based real-time embedded applications. IEEE Trans. Comput. 2011, 61, 1-31. [CrossRef]

9. Dorsey, J.; Searles, S.; Ciraula, M.; Johnson, S.; Bujanos, N.; Wu, D.; Braganza, M.; Meyers, S.; Fang, E.; Kumar, R. An integrated quad-core opteron ${ }^{\mathrm{TM}}$ processor. In Proceedings of the International Solid-Sate Circuits Conference, San Francisco, CA, USA, 11-15 February 2007; pp. 102-103.

10. Pakbaznia, E.; Pedram, M. Design and application of multimodal power gating structures. In Proceedings of the International Symposium on Quality Electronics Design, San Jose, CA, USA, 16-18 March 2009; pp. 120-126.

11. Cai, Q.; Gonzalez, J.; Magklis, G.; Chaparro, P.; Gonalez, A. Thread shuffling: Combining DVFS and thread migration to reduce energy consumptions for multi-core systems. In Proceedings of the IEEE/ACM International Symposium on Low Power Electronics and Design, Fukuoka, Japan, 1-3 August 2011; pp. 379-384.

12. Cao, A.; Sirisantana, N.; Koh, C.; Roy, K. Synthesis of selected clocked skewed logic circuits. In Proceedings of the International Symposium on Quality Electronic Design, San Jose, CA, USA, 18-21 March 2002; pp. 229-234.

13. Baker, R. CMOS: Circuit Design, Layout, and Simulation, 3rd ed.; Wiely: New York, NY, USA, 2011.

14. Fant, K.; Brandt, S. NULL convention logic: A complete and consistent logic for asynchronous digital circuit synthesis. In Proceedings of the International Conference on Application Specific Systems, Architectures, and Processors, Chicago, IL, USA, 19-23 August 1996; pp. 261-273.

15. Lucarz, C.; Mattavelli, M.; Dubois, J. A co-design platform for algorithm/architecture design exploration. In Proceedings of the International Conference on Control Systems and Computer Science, Hanoi, Vietnam, 17-20 December 2008; pp. 1069-1072.

16. Di, J.; Yuan, J.S. Energy-aware design for multi-rail encoding using NCL. IEEE Proc. Circuits Devices Syst. 2006, 153, 100-106. [CrossRef]

17. Di, J.; Bell, B.; Bouillon, W.; Brady, J.; Le, T.; Lo, C.; Men, L.; Nelson, S.; Sabado, F.; Suchanek, A. Recent advances in low power asynchronous circuit design. J. Low Power Electron. 2017, 13, 280-297. [CrossRef]

18. Min, A.; Wang, R.; Tsai, J.; Ergin, M.; Tai, T. Improving energy efficiency for mobile platforms by exploiting low-power sleep states. In Proceedings of the 9th Conference on Computing Frontiers, Cagliari, Italy, 15-17 May 2012.

19. Lin, J.; Yuan, J.S. A $300 \mathrm{mV}$, 6-bit ultra-low power SAR ADC. In Proceedings of the 2016 13th IEEE International Conference on Solid-State and Integrated Circuit Technology (ICSICT), Hangzhou, China, 25-28 October 2016; pp. 713-715.

20. Murmann, B. A/D converter trends: Power dissipation, scaling and digitally assisted architectures. In Proceedings of the IEEE Custom Integrated Circuits Conference, San Jose, CA, USA, 21-24 September 2008; pp. 105-112.

21. Gandhi, R.; Chen, Z.; Singh, N.; Banerjee, K.; Lee, S. CMOS-compatible rertical-silicon-nanowire gate-all-around p-type tunneling FETs with $\leq 50-\mathrm{mV} /$ decade subthreshold swing. IEEE Electron. Device Lett. 2011, 32, 1504-1506. [CrossRef]

22. Sedighi, B.; Hu, X.; Liu, H.; Nahas, S.J.; Niemieer, M. Analog circuit design using tunnel-FETs. IEEE Trans. Circuits Syst. I Regul. Pap. 2015, 62, 39-48. [CrossRef]

23. Lin, J.; Yuan, J. Ultra-low power successive approximation analog-to-digital converter using emerging tunnel field effect transistor technology. J. Low Power Electron. 2016, 12, 218-226. [CrossRef]

24. Murmann, B. ADC Performance Survey 1997-2015. Available online: http:/ / web.Stanford.edu/ murmann/ adcsurvey.html (accessed on 1 June 2016). 
25. Chen, Z.; Miyahara, M.; Matsuzawa, A. A 9.35-ENOB, 14.8 fJ/conv-step fully-passive noise-shaping SAR ADC. In Proceedings of the IEEE Symposium on VLSI Circuits, Kyoto, Japan, 17-19 June 2015; pp. C64-C65.

26. Guo, W.; Sun, N. A 12b-ENOB $61 \mu \mathrm{W}$ noise-shaping SAR ADC with a passive integrator. In Proceedings of the European Solid-State Circuits Conference, Toyama, Japan, 7-9 November 2016; pp. 405-408.

27. Schreier, R.; Temes, G.C. The second order delta sigma modulator. In Understanding Delta-Sigma Data Converters, 1st ed.; Wiley-IEEE Press: New York, NY, USA, 2005; pp. 63-90.

28. Lin, J.; Yuan, J. 12-bit ultra-low voltage noise shaping SAR ADC using emerging TFETs. J. Low Power Electron. 2017, 13, 497-510. [CrossRef]

29. Colli, A.; Pisana, S.; Fasoli, A.; Roberson, J.; Ferrari, A. Electronic transport in ambipolar silicon nanowires. Phys. Status Solidi 2007, 244, 4161-4164. [CrossRef]

30. Martel, R.; Deryche, V.; Lavoie, C.; Appenzeller, J.; Chan, K.; Tersoff, J.; Avouris, P. Ambipolar electrical transport in semiconducting single-wall carbon nanotubes. Phys. Rev. Lett. 2001, 87, 256805. [CrossRef] [PubMed]

31. Geim, A.; Novoselov, K. The rise of grapheme. Nat. Mater. 2007, 6, 183-191. [CrossRef] [PubMed]

32. Lin, Y.-M.; Appenzeller, J.; Knoch, J.; Avouris, P. High-performance carbon nanotube field-effect transistor with tunable polarities. IEEE Trans. Nanotechnol. 2005, 4, 451-489. [CrossRef]

33. Appenzeller, J.; Knoch, J.; Tutuc, E.; Reuter, M.; Guha, S. Dual-gate silicon nanowire transistors with nickel silicide contact. In Proceedings of the International Electron Devices Meeting, San Francisco, CA, USA, 11-13 December 2006; pp. 1-4.

34. Heinzig, A.; Slesazeck, S.; Freupl, F.; Mikolajick, T.; Weber, W. Reconfigurable silicon nanowire transistors. Nano Lett. 2012, 12, 1-119. [CrossRef] [PubMed]

35. Harada, N.; Yagi, K.; Sato, S.; Yokoyama, N. A polarity-controllable graphene inverter. Appl. Phys. Lett. 2010, 96, 012102. [CrossRef]

36. De Marchi, M.; Saccetto, D.; Frache, S.; Zhang, J.; Gaillardon, P.-E.; Leblebici, Y.; De Micheli, G. Polarity control in double-gate, gate-all-around vertically stacked silicon nanowire FETs. In Proceedings of the IEEE International Electron Devices Meeting, San Francisco, CA, USA, 10-13 December 2012.

37. Gaillardon, P.-E.; Bobba, S.; De Marchi, M.; Saccetto, D.; De Micheli, G. Nanowire systems: Technology and design. Philos. Trans. R. Soc. Lond. A 2014, 372. [CrossRef] [PubMed]

38. Seabaugh, A.; Zhang, Q. Low-voltage tunnel transistors for beyond CMOS logic. Proc. IEEE 2010, 98, 2095-2110. [CrossRef]

39. Lu, H.; Seabaugh, A. Tunnel field-effect transistors: State-of-the-art. IEEE J. Electron Devices Soc. 2014, 2, 44-49. [CrossRef]

40. Zhao, P.; Feenstra, R.; Gu, G.; Jena, D. SymFET: A proposed symmetric graphene tunneling field-effect transistor. IEEE Trans. Electron Devices 2013, 60, 951-957. [CrossRef]

41. Britnell, L.; Gorbachev, R.; Geim, A.; Ponomarenko, L.; Mishchenko, A.; Greenaway, M.; Fromhold, T.; Novoselov, K.; Eaves, L. Resonant tunneling and negative differential conductance in grapheme transistors. Nat. Commun. 2013, 4, 1794. [CrossRef] [PubMed]

42. Sedighi, B; Hu, X.; Nahas, J.; Niemier, M. Nontraditional computation using beyond-CMOS tunneling devices. IEEE J. Emerg. Sel. Top. Circuits Syst. 2014, 4, 438-449. [CrossRef]

43. Sedighi, B.; Hu, X.; Nahas, J.; Niemier, M. Boolean circuit design using emerging tunneling devices. In Proceedings of the International Conference on Computer Design, Dubai, UAE, 22-23 August 2014; pp. 355-360.

44. Kao, K.; Verhulst, A.; Vandenberghe, W.; Soree, B.; Groesneken, G.; Meyer, K. Direct and indirect band-to-band tunneling in germanium-based TFETs. IEEE Trans. Electron Devices 2012, 59, 292-301. [CrossRef]

45. Landau, L.; Lifschitz, E. Statistical Physics; Pergamon Press: Oxford, UK, 1980; Volume 6.

46. Khan, A.; Bhowmik, D.; Yu, P.; Kim, S.; Pan, X.; Ramesh, R.; Salahuddin, S. Experimental evidence of ferroelectric negative capacitance in nanoscale heterostructures. Appl. Phys. Lett. 2011, 99, 113501. [CrossRef]

47. DasGupta, S.; Rajashekhar, A.; Majumdar, K.; Agrawal, N.; Razavieh, A.; Trolier-McKinstry, S.; Datta, S. Sub-kT/q switching in strong inversion in $\mathrm{PbZr}_{0.52} \mathrm{Ti}_{0.48} \mathrm{O}_{3}$ gated negative capacitance FETs. IEEE J. Explor. Solid State Comput. Devices Circuits 2015, 1, 43-48. [CrossRef] 
48. Frougier, J.; Shukla, N.; Deng, D.; Jerry, M.; Aziz, A.; Liu, L.; Lavallee, G.; Mayer, T.S.; Gupta, S.; Datta, S. Phase-transition-FET exhibiting steep switching slope of $8 \mathrm{mV} /$ decade and $36 \%$ enhanced ON current. In Proceedings of the 2016 Symposium on VLSI Technology, Honolulu, HI, USA, 14-16 June 2016; pp. 228-229.

49. Huang, P.; Chen, S.; Zhao, Y.; Chen, B.; Gao, B.; Liu, L.; Chen, Y.; Zhang, Z.; Bu, W.; We, H.; et al. Self-selection RRAM cell with sub-uA switching current and robust reliability fabricated by high-k/metal gate CMOS compatible technology. IEEE Trans. Electron Devices 2016, 63, 4295-4301. [CrossRef]

50. Sandisk. Available online: https://www.rram-info.com/sandisk (accessed on 20 July 2017).

51. Raoux, S.; Burr, G.; Breitwisch, M.; Rettner, C.; Chen, Y.; Shelby, R.; Salinga, M.; Krebs, D.; Chen, S.; Lung, H. Phase-change random access memory: A scalable technology. IBM J. Res. Dev. 2010, 52, 465-479. [CrossRef]

52. Numonyx. The Basics of Phase Change Memory (PCM) Technology. 2008. Available online: http:/ / www. numonyx.com/Documents/WhitePapers /PCM_Basics_WP.pdf (accessed on 20 July 2017).

53. Xie, Y. Modeling, architecture, and applications for emerging memory technologies. IEEE Des. Test Comput. 2011, 28, 44-51. [CrossRef]

54. PR Newswire: Press Release Distribution, Targeting, Monitoring and Marketing. Available online: https: / www.prnewswire.com/news.releases/ibm-scientists-achieve-storage-memory-breakthrough300269117.html (accessed on 21 July 2017).

55. Seong, N.; Woo, F.; Lee, H. Security refresh: Prevent malicious wear-out and increase durability for phase-change memory with dynamically randomized address mapping. In Proceedings of the International Symposium on Computer Architecture, Saint-Malo, France, 19-23 June 2010; pp. 383-394.

56. Ban, A.; Hasharon, R. Wear leveling of static areas in flash memory. U.S. Patent Number 6,732,221, 4 May 2004.

57. Augustine, C.; Mojumder, N.; Fong, X.; Choday, S.; Park, S.; Roy, K. Spin-transfer torque MRAMs for low power memories: Perspective and prospective. IEEE Sens. J. 2012, 12, 756-766. [CrossRef]

58. Li, J.; Ndai, P.; Goel, A.; Salahuddin, S.; Roy, K. Design paradigm for robust spin-torque transfer magnetic RAM (STT MRAM) from circuit/architecture perspective. IEEE Trans. Very Large Scale Integr. VLSI Syst. 2010, 18, 1710-1723. [CrossRef]

59. Debrosse, J.; Gogl, D.; Bette, A.; Hoenigschmid, H.; Robertazzi, R.; Arndt, C.; Braun, D.; Casarotto, D.; Havreluk, R.; Lammers, S.; et al. A high-speed 128 Kbit MRAM core for future universal memory applications. In Proceedings of the IEEE International Symposium on VLSI Circuits, Kyoto, Japan, 12-14 June 2003; pp. 217-220.

60. Spin-Torque MRAM Technology. Available online: https://www.everspin.com/spin-torque-mramtechnology (accessed on 22 July 2017).

61. Parkin, S.; Hayashi, M.; Thomas, L. Magnetic domain-wall racetrack memory. Science 2008, 320, $190-194$. [CrossRef] [PubMed]

62. Annunziata, A.; Gaidis, M.; Thomas, L.; Chien, C.; Hung, C.; Chevalier, P.; Sullivan, E.; Hummel, J.; Joseph, E.; Zhu, Y.; et al. Racetrack memory cell array with integrated magnetic tunnel junction readout. In Proceedings of the International Electron Devices Meetings, Washington, DC, USA, 5-7 December 2011; pp. 539-542.

63. Venkatesan, R.; Kozhikkottu, V.; Augustine, C.; Raychowdhury, A.; Roy, K.; Raghunathan, A. Tapechach: A high density, energy efficient cache based on domain wall memory. In Proceedings of the International Symposium on Low Power Electronics and Design, Redondo Beach, CA, USA, 30 July-1 August 2012; pp. 185-190.

64. Venkatesan, R.; Sharad, M.; Roy, K.; Raghunathan, K. DWM-tapestri-An energy efficient all-spin cache using domain wall shift writes. In Proceedings of the Design, Automation \& Test Conference in Europe \& Exhibition, Grenoble, France, 18-22 March 2013; pp. 1825-1830.

65. Zhang, C.; Sun, G.; Zhang, W.; Mi, F.; Li, H.; Zhao, W. Quantitative modeling of racetrack memory, a tradeoff among area, performance, and power. In Proceedings of the Asia and South Pacific Design Automation Conference, Chiba, Japan, 19-22 January 2015; pp. 100-105.

66. Dery, H.; Dalal, P.; Cywinski, L.; Sham, L. Spin-based logic in semiconductors for reconfigurable large-scale circuits. Nature 2007, 447, 573-576. [CrossRef] [PubMed]

67. Augustine, C.; Panagopoulos, G.; Behin-Aein, B.; Srinivasan, S.; Sarkar, A.; Roy, K. Low-power functionality enhanced computation architecture using spin-based devices. In Proceedings of the IEEE/ACM International Symposium on Nanoscale Architectures, San Diego, CA, USA, 8-9 June 2011; pp. 129-136. 
68. Camsari, K.; Ganguly, S.; Datta, S. Modular approach to spintronics. Sci. Rep. Nat. 2015, 5, 10571. [CrossRef] [PubMed]

69. Kim, J.; Paul, A.; Crowell, P.; Koester, S.; Sapatnekar, S.; Wang, J.; Kim, H. Spin-based computing: Device concepts, current status, and a case study on a high-performance microprocessor. Proc. IEEE 2015, 103, 106-130.

70. Saripalli, V.; Sun, G.; Xie, Y.; Datta, S.; Narayanan, V. Exploiting heterogeneity for energy efficiency in chip multiprocessors. IEEE Trans. Emerg. Sel. Top. Circuits Syst. 2011, 1, 109-119. [CrossRef]

71. Guo, P.F.; Yang, L.T.; Yang, Y.; Fan, L.; Han, G.Q.; Samudra, G.S.; Yeo, Y.C. Tunneling field-effect transistor: Effect of strain and temperature on tunneling current. IEEE Electron Device Lett. 2009, 30, 981-983.

72. Lu, H.; Li, W.; Lu, Y.; Fay, P.; Ytterdal, T.; Seabaugh, A. Universal charge-conserving TFET SPICE model incorporating gate current and noise. IEEE J. Explor. Solid State Comput. Devices Circuits 2016, 2, $20-27$. [CrossRef]

73. Cadence Spectre Circuit Simulator. Available online: https://www.cadence.com/content/cadence-www/ global/en_US/home/tools/custom-ic-analog-rf-design/circuit-simulation/spectre-circuit-simulator. html (accessed on 27 May 2017).

74. Cao, Y.; Zhao, W. Predictive technology model for aano-CMOS design exploration. In Proceedings of the International Conference on Nano-Networks and Workshops, Lausanne, Switzerland, 14-16 September 2006; pp. 1-5.

75. Diehl, P.; Cook, M. Unsupervised learning of digit recognition using spike-timing-dependent plasticity. Front. Comput. Neurosci. 2015, 9, 1-8. [CrossRef] [PubMed]

76. Wu, X.; Saxena, V.; Zhu, K.; Balagopal, S. A CMOS spiking neuron for brain-inspired neural networks with resistive synapses and in-situ learning. IEEE Trans. Circuits Syst. II Express Br. 2015, 62, 1088-1092. [CrossRef]

77. Cassidy, A.; Sawada, J.; Merolla, P.; Arthur, J.; Alvarez-lcaze, R.; Akopyan, F.; Jackson, B.; Modha, D. TrueNorth: A high-performance, low-power neurosynaptic processor for multi-sensory perception, action, and cognition. In Proceedings of the Government Microcircuits Applications \& Critical Technology Conference, Orlando, FL, USA, 14-17 March 2016; pp. 341-344.

78. Cruz-Albrecht, J.; Yung, M.; Srinivasa, N. Energy-efficient neuron, synapse and STDP integrated circuits. IEEE Trans. Biomed. Circuits Syst. 2012, 6, 246-256. [CrossRef] [PubMed]

79. Naous, R.; Al-Shedivat, M.; Beftci, E.; Cauwenberghs, G.; Salama, K. Stochastic synaptic plasticity with memristor crossbar arrays. In Proceedings of the IEEE International Symposium on Circuits and Systems, Montréal, QC, Canada, 22-25 May 2016; pp. 2078-2081.

80. Srinivasan, G.; Sengupta, A.; Roy, K. Magnetic tunnel junction based long-term short-term stochastic synapse for a spiking neural network with on-chip STDP learning. Nature 2016, 6, 29545. [CrossRef] [PubMed]

81. Arias, O.; Wurm, J.; Hoang, K.; Jin, Y. Privacy and security in internet of things and wearable devices. IEEE Trans. Multi Scale Comput. Syst. 2015, 1, 99-109. [CrossRef]

82. Advanced Encryption Standard (AES), FIPS Pub 197. 2001. Available online: http://crsc.nist.gov/ publications / fips / fips197 / fips-197.pdf (accessed on 10 May 2017).

83. Ge, F.; Jain, R.; Choi, K. Ultra-Low power and high speed design and implementation of AES and SHA1 hardware cores in 65 nanometer CMOS technology. In Proceedings of the IEEE International Conference on Electro/Information Technology, Windsor, ON, Canada, 7-9 June 2009; p. 410.

84. Rivest, R.; Shamir, A.; Adleman, L. A method for obtaining digital signatures and public-key cryptosystems. IEEE Commun. ACM 1978, 21, 120-126. [CrossRef]

85. Tutanescu, I.; Anton, C.; Jonescu, L.; Caragata, D. Elliptic curves cryptosystems approaches. In Proceedings of the International Conference on Information Society, London, UK, 25-28 June 2012; pp. 357-362.

86. Gura, N.; Petal, A.; Wander, A.; Everle, H.; Shantz, S. Comparing elliptic curve cryptography and RSA on 8-bit CPUs. In Proceedings of the International Workshop on Cryptographic Hardware and Embedded Systems, Cambridge, MA, USA, 11-13 August 2004; pp. 925-943.

87. Leander, G.; Paar, C.; Poschmann, A.; Schramm, K. New lightweight des variants. In Fast Software Encryption; Birykov, A., Ed.; Springer: Berlin/Heidelberg, Germany, 2007; Volume 4593, pp. 196-210.

88. De Canniere, C.; Dunkelman, O.; Knezevic, M. KATAN \& KATANTAN-A family of small and efficient hardware-oriented block ciphers. In Proceedings of the International Workshop on Cryptographic Hardware and Embeded Systems, Lausanne, Switzerland, 6-9 September 2009; Springer: Berlin, Germany, 2009; pp. 272-288. 
89. Canniere, C.; Jaffe, J.; Jun, B. Differential power analysis. In Proceedings of the International Cryptology Conference on Advances on Cryptology, Santa Barbara, CA, USA, 15-19 August 1999; Wiener, M., Ed.; Springer: Berlin, Germany, 1999; pp. 388-397.

90. Kocher, P. Design and validation strategy for obtaining assurance in countermeasures to power analysis and related. In Proceedings of the NIST Physical Security Workshop, Honolulu, HI, USA, 26-29 September 2005.

91. Akkar, M.; Giraud, C. An implementation of DES and AES, secure against some attacks. In Proceedings of the Third International Workshop on Cryptographic Hardware and Embedded Systems, Paris, France, 14-16 May 2001; Springer: Berlin, Germany, 2001; Volume 2162, pp. 309-318.

92. Yang, S.; Wolf, W.; Vijaykrishnan, N.; Serpanos, D.; Xie, Y. Power attack resistant cryptosystem design: A dynamic voltage and frequency switching approach. In Proceedings of the Design, Automation \& Test Conference in Europe \& Exhibition, Washington, DC, USA, 7-11 March 2005; pp. 64-69.

93. Tiri, K.; Akmal, M.; Verbauwhede, I. A dynamic and differential CMOS logic with signal independent power consumption to withstand differential power analysis on smart cards. In Proceedings of the European Solid-State Circuits Conference, Firenze, Italy, 24-26 September 2002; pp. 403-406.

94. Bard, G.; Courtois, N.; Sepehrdad, J.; Zhang, B. Algebraic, aida/cube and side channel analysis of KATAN family of block ciphers. In Proceedings of the International Conference on Cryptology in India, Hyderabad, India, 12-15 December 2010; pp. 176-196.

95. Ralston, P.; Suko, S.; Fry, D.; Calatayud, R.; Kober, R. Development approach for supply chain hardware integrity for electronics defense (SHIELD) using ultra-small "dielets" with encryption and senor capability, near field powering and communications. In Proceedings of the Government Microcircuit Applications \& Critical Technology Conference, Orlando, FL, USA, 14-17 March 2016; pp. 97-100.

96. Rajendran, J.; Pino, Y.; Sinanoglu, O.; Karri, R. Logic encryption: A fault analysis perspective. In Proceedings of the 2012 Design, Automation \& Test in Europe Conference \& Exhibition (DATE), Dresden, Germany, 12-16 March 2012.

97. Chakraborty, R.; Bhunia, S. HARPOON: An obfuscation-based SoC design methodology for hardware protection. IEEE Trans. Comput. Aided Des. Integr. Circuits Syst. 2009, 28, 1493-1502. [CrossRef]

98. Yasin, M.; Rajendran, J.; Sinanoglu, O.; Karri, R. On improving the security of logic locking. IEEE Trans. Comput. Aided Des. Integr. Circuits Syst. 2016, 35, 1411-1424. [CrossRef]

99. Griffin, W.; Raghunathan, A.; Roy, K. CLIP: Circuit level IC protection through direct injection of process variations. IEEE Trans. Very Large Scale Integr. Syst. 2012, 20, 791-803. [CrossRef]

100. Alasad, Q.; Bi, Y.; Yuan, J. E²LEMI: Energy-efficient logic encryption using multiplexer insertion. Electronics 2017, 6, 16. [CrossRef]

101. Subramanyan, P.; Ray, S.; Malik, S. Evaluating the security of logic encryption algorithms. In Proceedings of the IEEE International Symposium on Hardware Oriented Security and Trust, Washington, DC, USA, 5-7 May 2015; pp. 137-143.

102. Xie, Y.; Srivastava, A. Mitigating SAT Attack on Logic Locking. Lecture Notes in Computer Science, Proceeding of the Cryptographic Hardware and Embedded Systems-CHES 2016, Santa Barbara, CA, USA, 17-19 August 2016; Springer: Berlin, Gemany, 2016; Volume 9813, pp. 127-146.

103. Yasin, M.; Mazumdar, B.; Rajendran, J.; Sinanoglu, O. SARLock: SAT attack resistant logic Locking. In Proceedings of the IEEE International Symposium on Hardware Oriented Security and Trust, McLean, VA, USA, 3-5 May 2016; pp. 236-241.

104. Yasin, M.; Mazumdar, B.; Sinanoglu, O.; Rajendran, J. Security analysis of anti-SAT. In Proceedings of the Asia and South Pacific Design Automation Conference, Chiba, Japan, 16-19 January 2017; pp. 342-347.

105. Alasa, Q.; Yuan, J.; Fan, D. Leveraging all-spin logic to improve hardware security. In Proceedings of the ACM Great Lake Symposium on VLSI, Banff, AB, Canada, 10-12 May 2017; pp. 491-494.

106. Imeson, F.; Emtenan, A.; Garg, S.; Tripunitara, M. Securing computer hardware using 3D integrated circuit (IC) technology and split manufacturing for obfuscation. In Proceedings of the USENIX Security Symposium, Washington, DC, USA, 14-16 August 2013; pp. 495-510.

107. Rajendran, J.; Sinanoglu, O.; Karri, R. Is split manufacturing secure? In Proceedings of the Design, Automation \& Test in Europe Conference \& Exhibition, Grenoble, France, 18-22 March 2013; pp. 1259-1264.

108. Vaidyanathan, K.; Das, B.; Sumbul, E.; Liu, R.; Pileggi, L. Building trusted ICs using split fabrication. In Proceedings of the IEEE International Symposium on Hardware-Oriented Security and Trust, Arlington, VA, USA, 6-7 May 2014. 
109. Vaidyanathan, K.; Liu, R.; Sumbul, E.; Zhu, Q.; Franchetti, F.; Pileggi, L. Efficient and secure intellectual property (IP) design with split fabrication. In Proceedings of the IEEE International Symposium on Hardware-Oriented Security and Trust, Arlington, VA, USA, 6-7 May 2014.

110. Jagasivamani, M.; Gadfort, P.; Sika, M.; Bajura, M.; Fritze, M. Split-fabrication obfuscation: Metrics and techniques. In Proceedings of the IEEE International Symposium on Hardware-Oriented Security and Trust, Arlington, VA, USA, 6-7 May 2014.

111. Hill, B.; Karmazin, R.; Otero, C.T.O.; Tse, J.; Manohar, R. A split-foundry asynchronous FPGA. In Proceedings of the Custom Integrated Circuits Conference, San Jose, CA, USA, 22-25 September 2013; pp. 1-4.

112. Xie, Y.; Bao, C.; Serafy, C.; Lu, T.; Srivastava, A.; Tehranipoor, M. Security and vulnerability implications of 3D ICs. IEEE Trans. Multi Scale Comput. Syst. 2016, 2, 108-122. [CrossRef]

113. Hunt, J.; Ding, Y.; Hsieh, A.; Chen, J.; Huang, D. Synergy between 2.5/3D development and hybrid 3D wafer level fanout. In Proceedings of the Electronic System-Integration Technology Conference, Amsterdam, The Netherlands, 17-20 September 2012; pp. 1-10.

114. Narasimhan, S.; Du, D.; Subhra, R.; Chakraborty, S.; Paul, S.; Wolff, F.; Papachristou, C.; Roy, K.; Bhunia, S. Hardware Trojan detection by multiple-parameter side-channel analysis. IEEE Trans. Comput. 2013, 62, 2183-2194. [CrossRef]

115. Van Woudenberg, J.; Witteman, M.; Bakker, B. Improving differential power analysis by elastic alignment. In Proceedings of the International Conference on Topics in Cryptology-CT-RSA 2011, San Francisco, CA, USA, 14-18 February 2011; Springer: Berlin, Germany; pp. 104-119.

116. Frontier Economics. Estimating the Global Economic and Social Impacts of Counterfeiting and Piracy; Technical Report; Frontier Economics Ltd.: London, UK, 2011.

117. Ronald, P.; James, P.; Bryan, J. Building Block for a Secure CMOS Logic Cell Library. U.S. Patent 20100301903 A1, 2 December 2010. Available online: http:/ / www.google.com/patents/US20100301903 (accessed on 10 June 2017).

118. Chow, L.; Baukus, J.; Wang, J.; Cocchi, R. Camouflaging a Standard Cell Based Integrated Circuit. U.S. Patent 8151235 B2, 3 April 2012. Available online: http://www.google.com/patents/US8151235 (accessed on 1 July 2017).

119. Rajendran, J.; Sinanoglu, O.; Sam, M.; Karri, R. Security analysis of integrated circuit camouflaging. In Proceedings of the ACM Conference on Computer and Communications Security, Berlin, Germany, 4-8 November 2013; pp. 709-720.

120. Stoica, A.; Zebulum, R.; Keymeulen, D.; Ferguson, M.; Duong, V. Taking evolutionary circuit design from experimentation to implementation: Some useful techniques and a silicon demonstration. IEE Proc. Comput. Digit. Tech. 2004, 151, 295-300. [CrossRef]

121. Ruzicka, R. New polymorphic NAND/XOR gate. In Proceedings of the International Conference on Applied Computer Science, Las Vegas, NV, USA, 25-28 June 2007; pp. 192-196.

122. Bi, Y.; Shamsi, K.; Yuan, J.-S.; Gaillardon, P.; De Micheli, G.; Yin, X.; Hu, X.; Niemier, M. Emerging technology-based design of primitives for hardware security. ACM J. Emerg. Technol. Comput. Syst. 2016, 13, 1-19. [CrossRef]

123. Alasad, B.; Jiann-Shiun Yuan, J.S.; Bi, Y. Logic Obfuscation against IC Reverse Engineering Attacks using Polymorphic Gates. In Proceedings of the IEEE International Conference on Computer Design, Boston, MA, USA, 5-8 November 2017; pp. 1-4.

124. Vatajelu, E.; Natale, G.; Torres, L.; Prinetto, P. STT-MRAM-based strong PUF architecture. In Proceedings of the IEEE Computer Society Annual Symposium on VLSI, Montpellier, France, 8-10 July 2015; pp. 467-472.

125. Zhang, L.; Fonf, X.; Chang, C.-H.; Kong, Z.; Roy, K. Highly reliable memory-based physical unclonable function using spin-transfer torque MRAM. In Proceedings of the IEEE International Symposium on Circuits and Systems, Melbourne, VIC, Australia, 1-5 June 2014; pp. 2069-2172.

126. Oosawa, S.; Konishi, T.; Onizawa, N.; Hanyu, T. Design of an STT-MTJ based true random number generator using digitally controlled probability-locked loop. In Proceedings of the IEEE International New Circuits and Systems Conference, Grenoble, France, 7-10 June 2015; pp. 1-4.

127. Kannan, S.; Karimi, N.; Sinanoglu, O.; Karri, R. Security vulnerabilities of emerging nonvolatile main memories and countermeasures. IEEE Trans. Comput. Aided Des. Integr. Circuits Syst. 2014, 34, 2-15. [CrossRef] 
128. Wang, Y.; Cai, H.; de Barros Naviner, L.; Zhang, Y.; Zhao, X.; Deng, E.; Klein, J.; Zhao, W. Compact model of dielectric breakdown in spin-transfer torque magnetic tunnel junction. IEEE Trans. Electron Devices 2016, 63, 1762-1767. [CrossRef]

129. Ikeda, S.; Hayakawa, J.; Lee, Y.; Matsukura, F.; Ohno, Y.; Hanyu, T.; Ohno, H. Magnetic tunnel junctions for spintronic memories and beyond. IEEE Trans. Electron Devices 2007, 54, 991-1002. [CrossRef]

130. Nikoubin, T.; Bahrebar, P.; Pouri, S.; Navi, K.; Iravani, V. Simple exact algorithm for transistor sizing of low-power high-speed arithmetic circuits. VLSI Des. 2010, 2010. [CrossRef]

131. Gandhi, D. Methods for Designing Standard Cell Transistor Structures. U.S. Patent 6,477,695, 5 November 2002.

132. Taheri, S.; Yuan, J. Security analysis of computing systems from circuit-architectural perspective. In Proceedings of the IEEE International Conference on Dependable and Secure Computing, Yaroslavl, Russia, 20-24 April 2017.

133. Zhou, P.; Zhao, B.; Yang, J.; Zhang, Y. A durable and energy efficient main memory using phase change memory technology. In Proceedings of the International Symposium on Computer Architecture, Austin, TX, USA, 20-24 June 2009; pp. 14-23.

134. Chhabra, S.; Solihin, Y. iNVMM: A secure non-volatile main memory system with incremental encryption. In Proceedings of the International Symposium on Computer Architecture, San Jose, CA, USA, 4-8 June 2011; pp. 177-188.

135. Kong, J.; Zhou, H. Improving privacy and lifetime of PCM-based main memory. In Proceedings of the International Conference on Dependable Systems and Networks, Chicago, IL, USA, 28 June-1 July 2010; pp. 333-342.

136. Lee, B.; Ipek, E.; Mutlu, O.; Burger, D. Architecting phase change memory as a scalable DRAM alternative. Comput. Archit. News 2009, 37, 2-13. [CrossRef]

137. Zhang, X.; Zhang, C.; Sun, G.; Di, J.; Zhang, T. An efficient run-time encryption scheme for non-valatile main memory. In Proceedings of the International Conference on Compilers, Architecture and Synthesis for Embedded Systems, Montreal, QC, Canada, 29 September-4 October 2013; pp. 1-10.

138. Xia, F.; Jiang, D.; Xiaong, J.; Sun, N. Write-aware random page initialization for non-volatile memory systems. In Proceedings of the IEEE International Conference on Computer Design, Seoul, Korea, 19-22 October 2014; pp. 208-215.

139. Qureshi, M.; Franchescini, M.; Srinivasan, V.; Lastras, L.; Abali, B.; Karidis, J. Enhancing lifetime and security of PCM-based main memory with start-gap wear leveling. In Proceedings of the IEEE/ACM International Symposium on Microarchitecture, New York, NY, USA, 12-16 December 2009; pp. 14-23.

140. Wu, G.; Zhang, H.; Dong, Y.; Hu, J. CAR: Securing PCM main memory system with cache address remapping. In Proceedings of the IEEE International Conference on Parallel and Distributed Systems, Singapore, 17-19 December 2012; pp. 626-635.

141. Qureshi, M.; Seznec, A.; Lastras, L.; Franceschini, M. Practical and secure PCM systems by online detection. In Proceedings of the 2011 IEEE 17th International Symposium on High Performance Computer Architecture, San Antonio, TX, USA, 12-16 February 2011; pp. 478-489.

142. Yu, H.; Du, Y. Increasing endurance and security of phase-change memory with multi-way wear-leveling. IEEE Trans. Comput. 2014, 63, 1157-1168.

143. Young, V.; Nair, P.; Quershi, M. DEUCE: Write-efficient encryption for non-volatile memories. In Proceedings of the ACM International Conference on Architectural Support for Programming Languages and Operating Systems, Istanbul, Turkey, 14-18 March 2015; pp. 33-44.

144. Mao, H.; Zhang, X.; Sun, G.; Sun, J. Protect non-volatile memory from wear-out attack based on timing difference of row buffer hit/miss. In Proceedings of the Design, Automation \& Test in Europe Conference \& Exhibition, Lausanne, Switzerland, 27-31 March 2017; pp. 1623-1626.

145. Yang, K.; Hicks, M.; Dong, H.; Austin, T.; Sylvester, D. A2: Analog malicious hardware. In Proceedings of the IEEE Symposium on Security and Privacy, San Jose, CA, USA, 22-26 May 2016; pp. 18-37.

146. Deyati, S.; Muldrey, B.; Chatterjee, A. Targeting hardware Trojans in mixed-signal circuits for security. In Proceedings of the IEEE International Mixed-Signal Testing Workshop, Sant Feliu de Guixols, Spain, 4-6 July 2016; pp. 1-4.

147. Bellizia, D.; Scotti, G.; Trifiletti, A. On-chip analog current equalizer as a countermeasure against side-channel attacks in CMOS nanometer technology. In Proceedings of the International Conference on Mixed Design of Integrated Circuits and System, Lodz, Poland, 23-25 June 2016; pp. 229-234. 
148. Jin, Y.; Markris, Y. Hardware Trojans in wireless cryptographic integrated circuits. IEEE Des. Test Comput. 2010, 27, 10-25. [CrossRef]

149. Wang, X.; Mal-Sarkar, T.; Krishna, A.; Narasimhan, S.; Bhunia, S. Software exploitable hardware Trojans in embedded processor. In Proceedings of the IEEE International Symposium on Defect and Fault Tolerance in VLSI and Nanotechnology Systems, Austin, TX, USA, 3-5 October 2012; pp. 55-58.

150. Rajendran, J.; Sinanoglu, O.; Karri, R. Regaining trust in VLSI design: Design-for-trust techniques. Proc. IEEE 2014, 102, 1266-1282. [CrossRef]

151. Taheri, S.; Lin, J.; Yuan, J. Security interrogation and defense for SAR analog to digital converter. Electronics 2017, 6, 48. [CrossRef]

152. Yang, C.; Liu, B.; Li, H.; Chen, Y.; Wen, W.; Barnell, M.; Wu, Q.; Wen, W.; Rajendran, J. Security of neuromorphic computing: Thwarting learning attacks using memristor's obsolescence effect. In Proceedings of the 2016 IEEE/ACM International Conference on Computer-Aided Design (ICCAD), Austin, TX, USA, 7-10 November 2016; pp. 1-6.

(C) 2017 by the authors. Licensee MDPI, Basel, Switzerland. This article is an open access article distributed under the terms and conditions of the Creative Commons Attribution (CC BY) license (http:// creativecommons.org/licenses/by/4.0/). 